TITLE:

\title{
Strichartz Estimates for Schrödinger Equations on Scattering Manifolds
}

$\operatorname{AUTHOR}(S)$ :

Mizutani, Haruya

\section{CITATION:}

Mizutani, Haruya. Strichartz Estimates for Schrödinger Equations on Scattering Manifolds. Communications in Partial Differential Equations 2012, 37(2): 169-224

ISSUE DATE:

2012-01

URL:

http://hdl.handle.net/2433/169704

\section{RIGHT:}

(C) Taylor \& Francis Group, LLC; This is not the published version. Please cite only the published version.; この論文は出版社版でありません。引 用の際には出版社版をご確認ご利用ください。 


\title{
STRICHARTZ ESTIMATES FOR SCHRÖDINGER EQUATIONS ON SCATTERING MANIFOLDS
}

\author{
HARUYA MIZUTANI
}

\begin{abstract}
The present paper is concerned with Schrödinger equations on non-compact Riemannian manifolds with asymptotically conic ends. It is shown that, for any admissible pair (including the endpoint), local in time Strichartz estimates outside a large compact set centered at origin hold. Moreover, we prove global in space Strichartz estimates under the nontrapping condition on the metric.
\end{abstract}

\section{Introduction}

Let us recall that the Strichartz estimates for the free Schrödinger equation on the Euclidean space $\mathbb{R}^{d}([21,10,23,14])$ state that

$$
\left\|e^{i \frac{t}{2} \Delta} u_{0}\right\|_{L^{p}\left([-T, T] ; L^{q}\left(\mathbb{R}^{d}\right)\right)} \leq C_{T}\left\|u_{0}\right\|_{L^{2}\left(\mathbb{R}^{d}\right)}, \quad u_{0} \in L^{2}\left(\mathbb{R}^{d}\right),
$$

where the pair $(p, q)$ satisfies the following admissible condition:

$$
\frac{2}{p}+\frac{d}{q}=\frac{d}{2}, \quad p, q \geq 2, \quad(d, p, q) \neq(2,2, \infty) .
$$

It is well known that these estimates are fundamental in studying low regularity well-posedness of the Cauchy problem for nonlinear Schrödinger equations. It is a natural question if the same estimates hold for Schrödinger equations on manifolds. Though the global in time estimates (i.e., $\left.\sup _{T} C_{T}<\infty\right)$ do not hold in general, the local in time estimates have been proved by many authors under several geometric conditions. The purpose of the present paper is to prove sharp Strichartz estimates on scattering manifolds for any admissible pair (including the endpoint $\left.\left(2, \frac{2 d}{d-2}\right)\right)$, where the scattering manifolds are non-compact manifolds which have asymptotically conic ends. The paper is also concerned with studying a relationship between Strichartz estimates and microlocal properties of the solution. More precisely, we show that (local in time) Strichartz estimates follow from the dispersive estimates for spatially and frequency localized solutions which we call microlocal dispersive estimates.

We consider the following model. We mean by the scattering manifold a non-compact manifold with an asymptotically conic structure. Let $M$ be a non-compact Riemannian manifold of dimension $d \geq 2$ such that $M$ can be decomposed as

$$
M=M_{c} \cup M_{\infty},
$$

where $M_{c} \Subset M$ is relatively compact, and there exists a $(d-1)$-dimensional closed Riemannian manifold $\partial M$ such that $M_{\infty}$ is diffeomorphic to $(0, \infty) \times \partial M$. Let

$$
\iota: M_{\infty} \ni z \mapsto(r(z), \theta(z)) \in(0, \infty) \times \partial M
$$

be an identification mapping which is called a boundary decomposition. Suppose that $M_{c} \cap$ $M_{\infty} \subset(0,1) \times \partial M$ under this identification. Throughout the paper we fix a boundary decomposition $\iota$ and do not write it explicitly, and denote local coordinates on $(0, \infty) \times \partial M$ by $(r, \theta)$.

2000 Mathematics Subject Classification. Primary 35A40; Secondary 35B45.

Key words and phrases. Strichartz estimate; Microlocal dispersive estimate; Scattering manifold; Schrödinger equation.

The author was partly supported by JSPS Research Fellowships for Young Scientists 08J06914(2008-2010); GCOE 'Fostering top leaders in mathematics', Kyoto University. 
We next recall the definition of the scattering metric on $M$. Let $g$ be a Riemannian metric on $M$ such that, for sufficiently large $R_{M}>0, g$ takes the form

$$
g=d r^{2}+r^{2}\left(h_{j k}+a_{j k}\right) d \theta^{j} d \theta^{k} \quad \text { for } \quad(r, \theta) \in\left(R_{M}, \infty\right) \times \partial M,
$$

where $\left(h_{j k}\right)$ is the Riemannian metric on $\partial M$ and $\left(a_{j k}\right)$ is a smooth and real-valued tensor. Here we used the Einstein summation convention. We may assume that $R_{M}=1$ without loss of generality, and define the scattering region by $\widetilde{M}_{\infty}:=(1, \infty) \times \partial M$. We also assume throughout the paper that there exists $\mu>0$ such that for any $(l, \alpha) \in \mathbb{Z}_{+}^{d}$,

$$
\left|\partial_{r}^{l} \partial_{\theta}^{\alpha} a_{j k}(r, \theta)\right| \leq C_{l \alpha} r^{-\mu-l}, \quad(r, \theta) \in \widetilde{M}_{\infty} .
$$

Such a $g$ is said to be a long-range scattering metric (in normal form).

Let $\Delta_{g}$ be the Laplace-Beltrami operator associated to $g$ on $L^{2}(M)$ :

$$
\Delta_{g}=\frac{1}{G(z)} \partial_{z_{l}} G(z) g^{l m}(z) \partial_{z_{l}},\left(g^{l m}(z)\right)=\left(g_{l m}(z)\right)^{-1}, G(z)=\sqrt{\operatorname{det} g_{l m}(z)},
$$

and set $P=-\frac{1}{2} \Delta_{g}$, where $L^{p}(M):=L^{p}(M, G(z) d z), 1 \leq p \leq \infty$. In the setting we consider the Schrödinger equation:

$$
\left\{\begin{array}{c}
i \partial_{t} u(t)=P u(t), \quad t \in \mathbb{R}, \\
u(0)=u_{0} \in L^{2}(M) .
\end{array}\right.
$$

Since $P$ is essentially self-adjoint on $C_{0}^{\infty}(M)$ under the above condition, (1.4) has a unique solution $u(t)=e^{-i t P} u_{0}$ by the Stone theorem. The main result is the following.

Theorem 1.1 (Strichartz estimates near infinity). There exist a large compact subset $M_{c} \subset$ $K \subset M$ and $\chi_{K} \in C_{0}^{\infty}(M)$ with $\chi_{K} \equiv 1$ on $K$ such that

$$
\left\|\left(1-\chi_{K}\right) e^{-i t P} u_{0}\right\|_{L^{p}\left([0,1] ; L^{q}(M)\right)} \leq C\left\|u_{0}\right\|_{L^{2}(M)}, \quad u_{0} \in C_{0}^{\infty}(M),
$$

provided that $(p, q)$ satisfies the admissible condition (1.1).

Let $p(z, \xi)$ be the principal symbol of $P$. We say that $M$ is nontrapping if for any $\left(z_{0}, \xi^{0}\right) \in$ $T^{*} M$ with $\xi^{0} \neq 0$, the geodesic flow $\left(z\left(t, z_{0}, \xi^{0}\right), \xi\left(t, z_{0}, \xi^{0}\right)\right)$ generated by $H_{p}$ satisfies

$$
\left|z\left(t, z_{0}, \xi^{0}\right)\right| \rightarrow+\infty \text { as } t \rightarrow \pm \infty,
$$

where $H_{p}=\sum_{j=1}^{d}\left(\frac{\partial p}{\partial \xi_{j}} \frac{\partial}{\partial z^{j}}-\frac{\partial p}{\partial z^{j}} \frac{\partial}{\partial \xi_{j}}\right)$ is the Hamilton vector field associated to $p(z, \xi)$. If $M$ is nontrapping, then using local smoothing effects which follow from resolvent estimates proved by [7] (see also [8]), we obtain local in time Strichartz estimates (without loss of derivatives) for $\chi_{K} e^{-i t P} u_{0}$ (see Appendix A for more details). Combining with Theorem 1.1, we have the following:

Theorem 1.2 (Global in space estimates). Suppose that $M$ is a nontrapping scattering manifold. Then, for any $(p, q)$ satisfying (1.1), we have

$$
\left\|e^{-i t P} u_{0}\right\|_{L^{p}\left([0,1] ; L^{q}(M)\right)} \leq C\left\|u_{0}\right\|_{L^{2}(M)}, \quad u_{0} \in C_{0}^{\infty}(M) .
$$

Remark 1.3. (i) Let $T>0$. Since $e^{-i t P}$ is unitary on $L^{2}(M)$, the time interval $[0,1]$ in $(1.5)$ and (1.6) can be replaced by $[-T, T]$ provided that we replace the constant $C>0$ by some $C_{T}>0$ depending on $T$.

(ii) (Metrics in not normal form) Let $g$ be a more general Riemannian metric than in normal form such that $g$ takes the form

$$
g=\left(1+a^{1}\right) d r^{2}+r a_{j}^{2}\left(d r d \theta^{j}+d \theta^{j} d r\right)+r^{2}\left(h_{j k}+a_{j k}^{3}\right) d \theta^{j} d \theta^{k}
$$

on $(1, \infty) \times \partial M$, where $a^{N}(r, \theta)$ are smooth and real-valued tensors satisfying (1.3) with some $0<\mu_{N}<1$. Then, there exists a change of coordinates $\left(r^{\prime}, \theta^{\prime}\right)=(R(r, \theta), \Theta(r, \theta))$ such that, for some $0<\mu<\mu_{N}$,

$$
R(r, \theta)=r+O\left(r^{1-\mu}\right), \Theta(r, \theta)=\theta+O\left(r^{-\mu}\right) \text { as } r \rightarrow+\infty,
$$


and $g$ can be brought to a long-range metric in normal form as above. Hence the statements of Theorem 1.1 and Theorem 1.2 also hold for such a metric in not normal form. We refer to [11, Section 10.6] for more details.

(iii) (Potential perturbations) Let $V$ be a smooth and real-valued potential on $M$ of shortrange type i.e.,

$$
\left|\partial_{r}^{l} \partial_{\theta}^{\alpha} V(r, \theta)\right| \leq C_{k \alpha} r^{-1-\nu-l} \quad \text { for } \quad(r, \theta) \in(1, \infty) \times \partial M,
$$

with some $\nu>0$. Our proof still works well if we replace $P$ with $P+V$. Hence the statements of Theorem 1.1 and Theorem 1.2 are still hold for $P+V$.

The local in time Strichartz estimates on manifolds recently have been studied by many authors. Staffilani-Tataru [20], Robbiano-Zuily [17] and Bouclet-Tzvetkov [4] studied the case of Schrödinger equations on the Euclidean space with the asymptotically flat metric under several settings. In [6], Burq-Gérard-Tzvetkov proved Strichartz estimates with a loss of derivative $1 / p$ on any compact manifolds without boundaries. They also proved that the loss $1 / p$ is optimal in the case of $M=\mathbb{S}^{d}$. Hassell-Tao-Wunsch [11] considered the case of nontrapping scattering manifolds except the endpoint estimate. Our result thus is regarded as a generalization of their result to the critical exponent case, however the method of the proof is considerably different. More recently, Bouclet $[1,2,3]$ studied the case of an asymptotically hyperbolic manifold which is a non-compact Riemannian manifold with the metric of the form $d r^{2}+e^{2 r}\left(h_{j k}+a_{j k}\right) d \theta^{j} d \theta^{k}$ in the scattering region, and he proved Strichartz estimates localized near infinity without the nontrapping condition. The present paper is motivated by his works. Global in time Strichartz estimates has been studied by $[5,22,16]$ in the case of Euclidean space with an asymptotically flat metric.

On the other hand, dispersive estimates for Schrödinger equations with potentials on the flat Euclidean space $\left(\mathbb{R}^{d}, \delta_{j k}\right)$ also have been studied by many authors. In particular, it was shown by Fujiwara [9] that if $V(x)$ is smooth, real-valued and increases at most quadratically at infinity, namely $\left|\partial_{x}^{\alpha} V(x)\right| \leq C_{\alpha}$ if $|\alpha| \geq 2$, then the fundamental solution $E(t, x, y)$ of the propagator $e^{-i t P}$ for $P=-\frac{1}{2} \Delta+V(x)$ satisfies the dispersive estimate $|E(t, x, y)| \leq C|t|^{-d / 2}$ on $\mathbb{R}^{d}$ provided that $t \neq 0$ is small enough. Local in time Strichartz estimates are immediate consequences of this estimate and the $T T^{*}$-argument due to [14]. Long time dispersive estimates for $e^{-i t P} P_{a c}(P)$, which implies global in time Strichartz estimates, also have been proved by many authors (e.g., Journé-Soffer-Sogge [13], Yajima [24, 25]) under suitable conditions of potentials and assumptions for the zero energy, where $P_{a c}(P)$ is the projection onto the absolutely continuous spectrum of $P$. For more references on dispersive estimates for Schrödinger equations with potentials, we refer to Schlag's survey [19].

The rest of the paper is devoted to the proof of Theorems 1.1 and 1.2. Though the proof is based on Bouclet's argument in [3], the behavior of classical trajectories at infinity $r \rightarrow$ $+\infty$ is different from the case of an asymptotically hyperbolic manifold and the class of the phase function of the parametrix becomes even worse. We thus cannot apply straightforwardly his method to the case of a scattering manifold. To overcome this difficulty, we introduce a localization in the $r$-variable by using the dyadic decomposition. The proof of Theorem 1.1 is then reduced to that of microlocal dispersive estimates.

The paper is organized as follows. In Section 2 we fix notations and the pseudodifferential setup, and collect results on the functional calculus recently proved by Bouclet $[1,2]$. Section 3 discusses a localization of both space and energy, and we show that Theorem 1.1 follows from microlocal dispersive estimates. We study some properties of the geodesic flow in Section 4. In Section 5 we construct the semiclassical Isozaki-Kitada parametrix and prove microlocal dispersive estimates on the strongly outgoing and incoming regions (cf. Definition 2.5). By using an Egorov type theorem, we prove microlocal smoothing properties of the propagator in Section 6 which imply microlocal dispersive estimates on intermediate regions. In Section 7, we construct the semiclassical WKB parametrix and prove short time microlocal dispersive estimates on the 
outgoing and incoming regions. We complete the proof of Theorem 1.1 in Section 8. We give the sketch of the proof of Theorem 1.2 in Appendix A.

Throughout the paper we use the following notations: We denote the set of multi-indices by $\mathbb{Z}_{+}^{d}$. For Banach spaces $X$ and $Y, \mathcal{L}(X, Y)$ denotes the Banach space of bounded operators from $X$ to $Y$, and we write $\mathcal{L}(X):=\mathcal{L}(X, X)$. For $a \in \mathbb{R}$, we use the notation $a_{+}=\max (a, 0)$. $\langle r\rangle$ stands for $\sqrt{1+|r|^{2}}$. For $A, B \geq 0, A \lesssim B$ means that there exists some universal constant $C>0$ such that $A \leq C B$.

Acknowledgments: The author would like to thank Jean-Marc Bouclet for helpful discussions and comments regarding the construction of the parametrix, in particular the spatial localization. He also would like to thank his advisor Shu Nakamura for much of comments and suggestions. He also thanks the anonymous referee for careful reading the manuscript and for giving useful comments.

\section{Preliminaries}

In this section we set up some standard notations on scattering manifolds. Notice that a boundary decomposition is always fixed. The first step is to choose a suitable atlas and a partition of unity on the scattering region $\widetilde{M}_{\infty}$. Let

$$
\left\{\kappa: \widetilde{M}_{\infty} \supset(1, \infty) \times V_{\kappa} \rightarrow(1, \infty) \times U_{\kappa} \subset \mathbb{R}^{d}\right\}_{\kappa}
$$

be a finite atlas on $\widetilde{M}_{\infty}$ such that $\kappa=\operatorname{Id} \otimes \kappa_{b}$, where $\left\{\kappa_{b}: \partial M \ni V_{\kappa} \rightarrow U_{\kappa} \Subset \mathbb{R}^{d-1}\right\}_{\kappa}$ is a finite atlas on $\partial M$. We denote the associated pull-back and push-forward by $\kappa^{*}$ and $\kappa_{*}=$ $\left(\kappa^{-1}\right)^{*}$, respectively. We also denote the induced chart diffeomorphism $T^{*}\left((1, \infty) \times V_{\kappa}\right) \rightarrow$ $T^{*}\left((1, \infty) \times U_{\kappa}\right) \cong(1, \infty) \times U_{\kappa} \times \mathbb{R}^{d}$ by the same symbol $\kappa_{*}$ if there is no confusion. Let $\left\{\psi_{\kappa_{b}}\right\}_{\kappa} \subset C_{0}^{\infty}\left(V_{\kappa}\right)$ be a partition of unity subordinate to $\left\{V_{\kappa}\right\}$ and $\psi \in C^{\infty}(\mathbb{R})$ a cut-off function such that $\operatorname{supp} \psi \subset(2, \infty)$ and $\psi \equiv 1$ for $r \geq 3$. We set $\psi_{\kappa}:=\psi \times \psi_{\kappa_{b}}$. Then, $\left\{\psi_{\kappa}\right\}_{\kappa}$ is a partition of unity subordinate to $\left\{(1, \infty) \times V_{\kappa}\right\}_{\kappa}$. Let $\tilde{\psi}_{\kappa} \in C^{\infty}\left(V_{\kappa}\right)$ be a cut-off function such that $\tilde{\psi}_{\kappa}$ takes the form $\tilde{\psi}_{\kappa}=\tilde{\psi} \times \tilde{\psi}_{\kappa_{b}}$, where $\tilde{\psi} \in C^{\infty}(\mathbb{R}), \tilde{\psi}_{\kappa_{b}} \in C_{0}^{\infty}\left(V_{\kappa}\right)$, supp $\tilde{\psi} \subset(3 / 2, \infty), \tilde{\psi} \equiv 1$ on $(2, \infty)$ and $\tilde{\psi}_{\kappa_{b}} \equiv 1$ close to $\operatorname{supp} \psi_{\kappa_{b}}$. Define smooth functions $\Psi_{\kappa}$ and $\tilde{\Psi}_{\kappa}$ on $(1, \infty) \times U_{\kappa}$ by $\Psi_{\kappa}=\kappa_{*} \psi_{\kappa}$ and $\tilde{\Psi}_{\kappa}=\kappa_{*} \tilde{\psi}_{\kappa}$, respectively. Then, $\left\{\Psi_{\kappa}\right\}_{\kappa}$ is a partition of unity subordinate to $\left\{(1, \infty) \times U_{\kappa}\right\}_{\kappa}$ and $\tilde{\Psi}_{\kappa}$ satisfies $\tilde{\Psi}_{\kappa}=1$ on $\operatorname{supp} \Psi_{\kappa}$.

2.1. Manifolds with asymptotically cylindrical ends. Noting that $r(z)$ can be extended to a positive smooth function on $M$ and is bounded on $M_{c}$ from above and below, we define a new density

$$
\widehat{G}(z) d z:=r(z)^{1-d} G(z) d z, z \in M,
$$

and set $L^{p}(\widehat{M}):=L^{p}(M, \widehat{G}(z) d z)$ for $1 \leq p<\infty, L^{\infty}(\widehat{M}):=L^{\infty}(M)$. (1.3) implies that

$$
\left|\partial_{r}^{l} \partial_{\theta}^{\alpha}\left(\widehat{G}(r, \theta)-\left(\operatorname{det} h_{j k}(\theta)\right)^{1 / 2}\right)\right| \leq C_{l \alpha} r^{-\mu-l} \text { for } r>1 \text {, }
$$

and that $\widehat{G}(r, \theta) d r d \theta$ is comparable with $d r d \theta$ for $r>1$. This fact implies that, for every function $u$ supported in $(1, \infty) \times V$ with $V \Subset V_{\kappa},\|u\|_{L^{p}(\widehat{M})}$ is equivalent to $\left\|\kappa_{*} u\right\|_{L^{p}\left(\mathbb{R}^{d}\right)}$ for all $1 \leq p \leq \infty$.

We next define an operator $\widehat{P}$ on $L^{2}(\widehat{M})$ by $\widehat{P}=r(z)^{\frac{d-1}{2}} \operatorname{Pr}(z)^{-\frac{d-1}{2}}$. It is easy to see that $\widehat{P}$ is unitarily equivalent to $P$ under the unitary map

$$
L^{2}(\widehat{M}) \ni u \mapsto r(z)^{-\frac{d-1}{2}} u \in L^{2}(M),
$$

and $\widehat{P}$ is essentially self-adjoint on $C_{0}^{\infty}(M)$. We denote the unique self-adjoint extension by the same symbol $\widehat{P}$. By definition, $(M, \widehat{G})$ can be regarded as a manifold with an asymptotically cylindrical end. For the most part of the paper we shall work with $\widehat{P}$ instead of $P$. By the formula (1.2) of the scattering metric $g, \widehat{P}_{\kappa}:=\kappa_{*} \widehat{P} \kappa^{*}$ takes the form

$$
\widehat{P}_{\kappa}=-\frac{1}{2} \widehat{G}^{-1}\left(\partial_{r}, \partial_{\theta} / r\right) \widehat{G}\left(\begin{array}{cc}
1 & 0 \\
0 & h+a
\end{array}\right)\left(\begin{array}{c}
\partial_{r} \\
\partial_{\theta} / r
\end{array}\right)+W, \quad(r, \theta) \in(1, \infty) \times U_{\kappa},
$$


where $h=\left(h^{j k}\right):=\left(h_{j k}\right)^{-1}$ and $a=\left(a^{j k}\right)$ are smooth and real-valued tensors, and $W$ is a smooth and real-valued potential such that

$$
\left|\partial_{r}^{l} \partial_{\theta}^{\alpha} a^{j k}(r, \theta)\right| \leq C_{k \alpha} r^{-\mu-l}, \quad\left|\partial_{r}^{l} \partial_{\theta}^{\alpha} W(r, \theta)\right| \leq C_{k \alpha} r^{-1-\mu-l} \quad \text { for } \quad r>1 .
$$

Denoting by $(\rho, \omega)$ the dual coordinate to the vector field $\left(\partial_{r}, \partial_{\theta}\right)$, the principal symbol of $\widehat{P}_{\kappa}$ is written in the form

$$
p_{\kappa}(r, \theta, \rho, \omega)=\frac{1}{2} \rho^{2}+\frac{1}{2 r^{2}}\left(h^{j k}(\theta)+a^{j k}(r, \theta)\right) \omega_{j} \omega_{k} \quad \text { on } \quad T^{*}\left((1, \infty) \times U_{\kappa}\right) .
$$

Moreover, the full symbol of $\widehat{P}_{\kappa}$ takes the form $p_{\kappa}+p_{\kappa 1}+p_{\kappa 2}$, where $p_{\kappa j}$ can be written in the form

$$
p_{\kappa j}(r, \theta, \rho, \omega)=\sum_{k+|\beta|=2-j} b_{\kappa j}^{k \beta}(r, \theta) \rho^{k}(\omega / r)^{\beta},
$$

with some smooth functions $b_{\kappa j}^{k \beta}(r, \theta)$ on $(1, \infty) \times U_{\kappa}$ satisfying

$$
\left|\partial_{r}^{l} \partial_{\theta}^{\alpha} b_{\kappa j}^{k \beta}(r, \theta)\right| \leq C_{l \alpha k \beta} r^{-1-\mu-l} .
$$

2.2. Pseudodifferential calculus on scattering manifolds. In this subsection we define pseudodifferential operators and study their properties. Moreover we collect known results on the functional calculus on scattering manifolds which were proved in [1] in a more general setting. We begin with the definition of our symbol class.

Definition 2.1. Let $X$ be an open subset of $T^{*}\left((1, \infty) \times \mathbb{R}^{d-1}\right)=(1, \infty)_{r} \times \mathbb{R}_{\theta}^{d-1} \times \mathbb{R}_{\rho} \times \mathbb{R}_{\omega}^{d-1}$ such that $\pi_{\theta}(X)$ is relatively compact, and that

$$
|\omega| \lesssim r \quad \text { in } X,
$$

where $\pi_{\theta}$ is the projection onto the $\theta$-space. We define the symbol class $S_{\mathrm{sc}}(X)$ as the set of all $a \in C^{\infty}\left(\mathbb{R}^{2 d}\right)$ such that $\operatorname{supp} a \subset X$, and that

$$
\left|\partial_{r}^{j} \partial_{\theta}^{\alpha} \partial_{\rho}^{k} \partial_{\omega}^{\beta} a(r, \theta, \rho, \omega)\right| \leq C_{j \alpha k \beta} r^{-j-|\beta|} \quad \text { on } \quad X .
$$

Example 2.2. Let $\tilde{U}_{\kappa} \Subset U_{\kappa}$ be an open subset with $\tilde{\Psi}_{\kappa}=1$ on $(2, \infty) \times \tilde{U}_{\kappa}, p_{\kappa}$ the principal symbol of $P_{\kappa}$ and $\varphi \in C_{0}^{\infty}((0, \infty))$. For $R \geq 2$ and an open interval $J \Subset(0, \infty)$ with $\operatorname{supp} \varphi \Subset J$, we set $X_{\kappa}=(R, \infty) \times \tilde{U}_{\kappa} \times \mathbb{R}^{d} \cap p_{\kappa}^{-1}(J)$. Since $|\rho|^{2}+|\omega / r|^{2} \leq C p_{\kappa}(r, \theta, \rho, \omega) \leq C \sup J, X_{\kappa}$ satisfies (2.4). Moreover, by (2.1) and (2.2), we have

$$
\left|\partial_{r}^{j} \partial_{\theta}^{\alpha} \partial_{\rho}^{k} \partial_{\omega}^{\beta} p_{\kappa}(r, \theta, \rho, \omega)\right| \leq C_{j \alpha k \beta} r^{-j-|\beta|}\left(1+|\rho|^{2}+|\omega / r|^{2}\right) .
$$

Therefore, $\varphi \circ p_{\kappa} \in S_{\mathrm{sc}}\left(X_{\kappa}\right) . \varphi \circ p_{\kappa}$ is the principal symbol of the semiclassical pseudodifferential approximation of $\varphi\left(h^{2} \widehat{P}\right)$ in the coordinated neighborhood $(1, \infty) \times U_{\kappa}$ (cf. Lemma 2.4).

Suppose that $X_{\kappa} \subset(1, \infty) \times U_{\kappa} \times \mathbb{R}^{d}$ satisfies (2.4) and $\tilde{\Psi}_{\kappa}=1$ near $\pi\left(X_{\kappa}\right)$, where $\pi$ : $T^{*} \mathbb{R}^{d} \rightarrow \mathbb{R}^{d}$ is the projection onto the base space. For all $h \in(0,1]$ and $a \in S_{\mathrm{sc}}\left(X_{\kappa}\right)$, we define the semiclassical pseudodifferential operator ( $h$-PDO for short) by

$$
\mathrm{Op}_{\kappa}(a) u:=\kappa^{*}\left(a\left(r, \theta, h D_{r}, h D_{\theta}\right) \kappa_{*}\left(\tilde{\Psi}_{\kappa} u\right)\right): C_{0}^{\infty}(M) \rightarrow C^{\infty}(M),
$$

where $a\left(r, \theta, h D_{r}, h D_{\theta}\right)$ is the standard $h$-PDO which has the kernel

$$
(2 \pi h)^{-d} \int_{\mathbb{R}^{d}} e^{\frac{i}{h}\left[\left(r-r^{\prime}\right) \rho+\left(\theta-\theta^{\prime}\right) \omega\right]} a(r, \theta, \rho, \omega) d \rho d \omega .
$$

Note that since $\pi(\operatorname{supp} a) \subset(2, \infty) \times \tilde{U}_{\kappa}$, for any $f_{\kappa} \in C^{\infty}(M)$ with $\kappa_{*} f_{\kappa} \equiv 1$ on $\pi(\operatorname{supp} a)$, we see that $\operatorname{Op}_{\kappa}(a) u=f_{\kappa} \mathrm{Op}_{\kappa}(a) u$. $\mathrm{Op}_{\kappa}(a)$ is thus well-defined on $M$. Morever, the CalderónVaillancourt theorem shows that $\operatorname{Op}_{\kappa}(a)$ extends to a bounded operator on $L^{2}(\widehat{M})$ and satisfies

$$
\left\|\mathrm{Op}_{\kappa}(a)\right\|_{\mathcal{L}\left(L^{2}(\widehat{M})\right)} \leq C_{d} \sum_{|\gamma| \leq M_{d}}\left\|\partial_{(r, \theta, \rho, \omega)}^{\gamma} a\right\|_{L^{\infty}\left(\mathbb{R}^{2 d}\right)}<\infty
$$

uniformly with respect to $h \in(0,1]$, where $C_{d}, M_{d} \geq 0$ depend only on $d$. 
We next describe basic symbolic calculus for $S_{\mathrm{sc}}\left(X_{\kappa}\right)$. We first note that (2.4) and (2.5) are invariant under coordinate transformations since any chart diffeomorphism $\kappa$ takes the form $\kappa=\operatorname{Id} \otimes \kappa_{b}$. Let $a \in S_{\mathrm{sc}}\left(X_{\kappa}\right)$ and $b \in S_{\mathrm{sc}}\left(X_{\kappa^{\prime}}\right)$. The above fact allow us to define the composition $\mathrm{Op}_{\kappa}(a) \circ \mathrm{Op}_{\kappa^{\prime}}(b)$, though $\mathrm{Op}_{\kappa^{\prime}}(b)$ is not a properly supported $h$-PDO (see Definition 2.3) in general. A standard symbolic calculus implies that the symbol of composition has the following semiclassical expansion:

$$
\sum_{l+|\alpha|=0}^{N} \frac{h^{l+|\alpha|}}{l ! \alpha !} \partial_{\rho}^{l} \partial_{\omega}^{\alpha} a(r, \theta, \rho, \omega) D_{r}^{l} D_{\theta}^{\alpha} \nu_{b *}\left(\tilde{\Psi}_{\kappa^{\prime}} b\right)(r, \theta, \rho, \omega)+h^{N+1} r_{N}(r, \theta, \rho, \omega),
$$

where $r_{N} \in S_{\mathrm{sc}}\left(X_{\kappa}\right)$ and $\nu_{b *}$ is the induced chart diffeomorphism with respect to $\nu_{b}=\kappa_{b}^{\prime} \circ \kappa_{b}^{-1}$. Note that if $V_{\kappa} \cap V_{\kappa^{\prime}}=\emptyset$, then $\mathrm{Op}_{\kappa}\left(a_{\kappa}\right) \circ \mathrm{Op}_{\kappa^{\prime}}\left(b_{\kappa^{\prime}}\right)=0$.

Following [1], we also define the properly supported $h$-PDO.

Definition 2.3. Let $\Psi \in C_{0}^{\infty}\left(\mathbb{R}^{d}\right)$ be a cut-off function such that $\operatorname{supp} \Psi \subset\{|z|<\delta\}$ and $\Psi \equiv 1$ on $\{|z|<\delta / 2\}$ with some small $\delta>0$. For $a \in S_{\text {sc }}\left(X_{\kappa}\right)$, we then define the properly supported $h$-PDO

$$
\mathrm{Op}_{\kappa}^{p r}(a): C_{0}^{\infty}(M) \rightarrow C_{0}^{\infty}(M)
$$

as the operator so that $\kappa_{*} \mathrm{Op}_{\kappa}^{p r}(a) \kappa^{*}$ has the kernel

$$
(2 \pi h)^{-d} \int e^{\frac{i}{h}\left[\left(r-r^{\prime}\right) \rho+\left(\theta-\theta^{\prime}\right) \omega\right]} a(r, \theta, \rho, \omega) \Psi\left(r-r^{\prime}, \theta-\theta^{\prime}\right) d \rho d \omega .
$$

When $\delta$ is small, $\Psi \equiv 1$ sufficiently near $\pi\left(X_{\kappa}\right)$ since supp $a \subset X_{\kappa}$. We hence removed the factor $\tilde{\Psi}_{\kappa}$ of the amplitude. We also note that $\operatorname{Op}_{h}^{p r}(a)$ is uniquely determined on $L^{2}(\widehat{M})$ up to $O\left(h^{\infty}\right)$. More precisely, we have for any $N \geq 0$,

$$
\left\|\mathrm{Op}_{\kappa}(a)-\mathrm{Op}_{\kappa}^{p r}(a)\right\|_{\mathcal{L}\left(L^{2}(\widehat{M})\right)} \leq C_{N} h^{N}
$$

uniformly with respect to $h \in(0,1]$ since $\operatorname{supp}(1-\Psi)$ away from the diagonal. We here describe a simple property of the properly supported $h$-PDO. Choose arbitrarily $\chi_{0}, \chi_{1} \in C_{0}^{\infty}((2, \infty))$ so that $\chi_{1} \equiv 1$ on $\left\{r \mid \operatorname{dist}\left(\operatorname{supp} \chi_{0}, r\right)<2 \delta\right\}$. We then have

$$
\chi_{0}(r) \Psi\left(r-r^{\prime}, \theta-\theta^{\prime}\right)=\chi_{0}(r) \Psi\left(r-r^{\prime}, \theta-\theta^{\prime}\right) \chi_{1}\left(r^{\prime}\right) .
$$

In particular,

$$
\chi_{0}(r) \mathrm{Op}_{\kappa}^{p r}(a)=\chi_{0}(r) \mathrm{Op}_{\kappa}^{p r}(a) \chi_{1}\left(r^{\prime}\right), \mathrm{Op}_{\kappa}^{p r}(a) \chi_{0}\left(r^{\prime}\right)=\chi_{1}(r) \mathrm{Op}_{\kappa}^{p r}(a) \chi_{0}\left(r^{\prime}\right) .
$$

This property plays an important role in the spatial localization.

Fix $\varphi \in C_{0}^{\infty}((0, \infty))$ and a relatively compact open interval $J \Subset(0, \infty)$ so that $\operatorname{supp} \varphi \Subset J$. Let $\chi \in C_{0}^{\infty}(M)$ be a smooth cut-off function such that $\chi(z)=1$ for $z \in M_{c} \cup \iota^{-1}\left(\left(0, R_{0}\right) \times \partial M\right)$, $\chi(z)=0$ for $z \in \iota^{-1}\left(\left(R_{0}+1, \infty\right) \times \partial M\right)$ with some $R_{0}>1$. By using above $h$-PDO's, we have two kinds of the semiclassical approximations of $(1-\chi) \varphi\left(h^{2} \widehat{P}\right)$.

Lemma 2.4 ([1]). Let $\delta>0$ be small enough. Then, for each $\kappa$ and all $N \geq 0$, there exist semiclassical symbols

$$
a_{\kappa, h}=\sum_{j=0}^{N} h^{j} a_{\kappa, j} \quad \text { with } \quad a_{\kappa, j} \in S_{\mathrm{sc}}\left(\left(R_{0}, \infty\right) \times \tilde{U}_{\kappa} \times \mathbb{R}^{d} \cap p_{\kappa}^{-1}(J)\right),
$$

such that

$$
(1-\chi) \varphi\left(h^{2} \widehat{P}\right)=\sum_{\kappa} \mathrm{Op}_{\kappa}\left(a_{\kappa, h}\right)+h^{N+1} R_{N}(h)=\sum_{\kappa} \mathrm{Op}_{\kappa}^{p r}\left(a_{\kappa, h}\right)+h^{N+1} R_{N}^{p r}(h)
$$

on $L^{2}(\widehat{M})$. Moreover, there exists $C_{N}>0$ such that the followings hold true uniformly with respect to $h \in(0,1]$ :

(i) $\left(L^{2}(\widehat{M})\right.$-boundedness $)$

$$
\left\|\left.\mathrm{Op}_{\kappa}\left(a_{\kappa, h}\right)\right|_{\mathcal{L}\left(L^{2}(\widehat{M})\right)}+\right\| R_{N}(h) \|_{\mathcal{L}\left(L^{2}(\widehat{M})\right)} \leq C_{N}
$$


(ii) (semiclassical Sobolev embedding) for $2 \leq q \leq \infty$,

$$
\begin{aligned}
\left\|r^{-\frac{d-1}{2}} \operatorname{Op}_{\kappa}^{p r}\left(a_{\kappa, h}\right)\right\|_{\mathcal{L}\left(L^{2}(\widehat{M}), L^{q}(M)\right)} & \leq C_{N} h^{-d(1 / 2-1 / q)}, \\
\left\|r^{-\frac{d-1}{2}} R_{N}^{p r}(h)\right\|_{\mathcal{L}\left(L^{2}(\widehat{M}), L^{q}(M)\right)} & \leq C_{N} h^{-d(1 / 2-1 / q)} ;
\end{aligned}
$$

(iii) (weighted $L^{q}(\widehat{M})$-boundedness) for $1 \leq q \leq \infty$ and all $s \in \mathbb{R}$,

$$
\left\|r^{-s} \mathrm{Op}_{\kappa}^{p r}\left(a_{\kappa, h}\right) r^{s}\right\|_{\mathcal{L}\left(L^{q}(\widehat{M})\right)} \leq C_{N} .
$$

Proof. The proof was essentially given by [1]. We hence only check that $a_{\kappa, j} \in S_{\mathrm{sc}}\left(\left(R_{0}, \infty\right) \times\right.$ $\left.\tilde{U}_{\kappa} \times \mathbb{R}^{d} \cap p_{\kappa}^{-1}(J)\right) . a_{\kappa, 0}$ is explicitly given by $a_{\kappa, 0}:=\kappa_{*}(1-\chi) \cdot \varphi \circ p_{\kappa}$. Moreover, for each $j$, $a_{\kappa, j}$ is of the form

$$
\sum_{k \leq N_{j}} d_{j k} \cdot\left(\partial^{k} \varphi\right) \circ p_{\kappa} \quad \text { for some } \quad 0<N_{j}<\infty .
$$

For each $k, d_{j k}$ is a polynomial of degree $2 k-j \geq 0$ with respect to $(\rho, \omega / r)$, and its coefficients are linear combinations of products of derivatives of $\tilde{\Psi}_{\kappa}, \kappa_{*}(1-\chi)$ and the full symbol of $P_{\kappa}$. Therefore, $a_{\kappa, j}$ takes the form

$$
a_{\kappa, j}(r, \theta, \rho, \omega)=b_{\kappa, j}(r, \theta, \rho, \omega / r),
$$

where $b_{\kappa, j}$ is compactly supported with respect to $\rho$ and $\omega$, and satisfies

$$
\left|\partial_{r}^{l} \partial_{\theta}^{\alpha} \partial_{\rho}^{m} \partial_{\omega}^{\beta} b_{\kappa, j}(r, \theta, \rho, \omega)\right| \leq C_{l \alpha k \beta R_{0}} r^{-l} .
$$

We hence obtain $a_{\kappa, j} \in S_{\mathrm{sc}}\left(\left(R_{0}, \infty\right) \times \tilde{U}_{\kappa} \times \mathbb{R}^{d} \cap p_{\kappa}^{-1}(J)\right)$.

2.3. Outgoing and incoming regions. In this subsection we recall the definition of the outgoing and incoming regions and study some basic properties of these regions needed later. Let $R \geq 1, \tilde{U}_{\kappa} \Subset U_{\kappa}$ an open subset, $J \Subset(0, \infty)$ an open interval and $\sigma \in(-1,1)$.

Definition 2.5. (i) We set

$$
\Gamma^{ \pm}\left(R, \tilde{U}_{\kappa}, J, \sigma\right)=\left\{(r, \theta, \rho, \omega) \in \mathbb{R}^{2 d} \mid r>R, \theta \in \tilde{U}_{\kappa}, p_{\kappa} \in J, \pm \rho>-\sigma \sqrt{2 p_{\kappa}}\right\},
$$

where $p_{\kappa}=p_{\kappa}(r, \theta, \rho, \omega) . \Gamma^{+}\left(R, \tilde{U}_{\kappa}, J, \sigma\right)$ (resp. $\left.\Gamma^{-}\left(R, \tilde{U}_{\kappa}, J, \sigma\right)\right)$ is said to be the outgoing (resp. incoming) region.

(ii) Let $\tilde{U}_{\kappa, \sqrt{\varepsilon}}$ and $J_{\varepsilon^{2}}$ be an $\sqrt{\varepsilon}$-neighborhood of $\tilde{U}_{\kappa}$ and an $\varepsilon^{2}$-neighborhood of $J$, respectively:

$$
\tilde{U}_{\kappa, \sqrt{\varepsilon}}:=\left\{\theta \in \mathbb{R}^{d-1} \mid \operatorname{dist}\left(\tilde{U}_{\kappa}, \theta\right)<\sqrt{\varepsilon}\right\}, \quad J_{\varepsilon^{2}}:=\left\{\rho \pm \varepsilon^{2} \in(0, \infty) \mid \rho \in J\right\} .
$$

For sufficiently small $\varepsilon>0$ such that $\tilde{U}_{\kappa, \sqrt{\varepsilon}} \Subset U_{\kappa}$, we define the strongly outgoing and incoming regions as follows:

$$
\Gamma_{s}^{ \pm}\left(R, \tilde{U}_{\kappa}, J, \varepsilon\right):=\Gamma^{ \pm}\left(R, \tilde{U}_{\kappa, \sqrt{\varepsilon}}, J_{\varepsilon^{2}},-\sqrt{1-\varepsilon^{2}}\right) .
$$

(iii) For sufficiently small $\varepsilon, \delta>0$, and for any $L>0$ and $\sigma_{l} \in(-1,1 / 2], l=0,2, \ldots, L$, satisfying

$$
\left\{\begin{array}{c}
\left(-\sqrt{1-\varepsilon^{2} / 4}, 1 / 2\right)=\bigcup_{l=1}^{L-1}\left(\sigma_{l-1}, \sigma_{l+1}\right), \\
-\sqrt{1-\varepsilon^{2} / 4}=\sigma_{0}<\sigma_{1}<\ldots<\sigma_{L}=1 / 2, \quad\left|\sigma_{l+1}-\sigma_{l-1}\right| \leq \delta,
\end{array}\right.
$$

the intermediate outgoing region and incoming region are defined by

$$
\begin{aligned}
& \Gamma_{i}^{ \pm}\left(R, \tilde{U}_{\kappa}, J, \varepsilon, \delta, l\right) \\
& :=\Gamma^{ \pm}\left(R, \tilde{U}_{\kappa, \sqrt{\varepsilon}}, J_{\varepsilon^{2}}, 1 / 2\right) \cap\left\{-\sigma_{l+1} \sqrt{2 p_{\kappa}}< \pm \rho<-\sigma_{l-1} \sqrt{2 p_{\kappa}}\right\} .
\end{aligned}
$$


We describe basic properties of these regions. $\Gamma^{ \pm}\left(R, \tilde{U}_{\kappa}, J, \sigma\right)$ are monotonously decreasing with respect to $R$, and increasing with respect to $\tilde{U}_{\kappa}, J$ and $\sigma$. By definition, we have

$$
(R, \infty) \times \tilde{U}_{\kappa} \times \mathbb{R}^{d} \cap p_{\kappa}^{-1}(J) \subset \bigcup_{ \pm} \Gamma^{ \pm}\left(R, \tilde{U}_{\kappa}, J, 1 / 2\right) .
$$

We also obtain

$$
\Gamma^{ \pm}\left(R, \tilde{U}_{\kappa}, J, 1 / 2\right) \subset \Gamma_{s}^{ \pm}\left(R, \tilde{U}_{\kappa}, J, \varepsilon\right) \cup \bigcup_{l=1}^{L-1} \Gamma_{i}^{ \pm}\left(R, \tilde{U}_{\kappa}, J, \varepsilon, \delta, l\right),
$$

respectively. Moreover for sufficiently large $R_{0}, C>0$, all $0<\varepsilon<1 / 2$ and $R \geq R_{0}$,

$$
|\omega / r| \leq C \varepsilon \quad \text { on } \quad \Gamma_{s}^{ \pm}\left(R, \tilde{U}_{\kappa}, J, \varepsilon\right)
$$

Indeed, since $\rho^{2} \geq\left(1-\varepsilon^{2}\right)\left(\rho^{2}+\frac{1}{r^{2}}\left(h^{j k}+a^{j k}\right) \omega_{j} \omega_{k}\right)$ on $\Gamma_{s}^{ \pm}\left(R, \tilde{U}_{\kappa}, J, \varepsilon\right)$, taking $R_{0}>0$ large enough so that $\left(h^{j k}+a^{j k}\right)_{j, k} \geq C_{0}^{-1}$ Id for some fixed $C_{0}>0$, we obtain $|\omega / r|^{2} \leq C_{0} \rho^{2} \varepsilon^{2} /\left(1-\varepsilon^{2}\right)$.

We also define spatial localized regions as follows.

Definition 2.6. Let $\varepsilon, \delta, L, \sigma_{l}$ be as above. For $R_{2}>R_{1}>1$, we define the spatial localized outgoing and incoming regions $\Omega^{ \pm}\left(R_{1}, R_{2}, \tilde{U}_{\kappa}, J, \sigma\right)$ by

$$
\Omega^{ \pm}\left(R_{1}, R_{2}, \tilde{U}_{\kappa}, J, \sigma\right):=\Gamma^{ \pm}\left(R_{1}, \tilde{U}_{\kappa}, J, \sigma\right) \cap\left\{R_{1}<r<4 R_{2}\right\} .
$$

We shall use the notation $\Omega^{ \pm}\left(R, \tilde{U}_{\kappa}, J, \sigma\right)=\Omega^{ \pm}\left(R, R, \tilde{U}_{\kappa}, J, \sigma\right)$. We also define the corresponding spatial localized strongly outgoing (incoming) and intermediate regions

$$
\Omega_{s}^{ \pm}\left(R_{1}, R_{2}, \tilde{U}_{\kappa}, J, \varepsilon\right), \Omega_{s}^{ \pm}\left(R, \tilde{U}_{\kappa}, J, \varepsilon\right), \Omega_{i}^{ \pm}\left(R_{1}, R_{2}, \tilde{U}_{\kappa}, J, \varepsilon, \delta, l\right),
$$

and $\Omega_{i}^{ \pm}\left(R, \tilde{U}_{\kappa}, J, \varepsilon, \delta, l\right)$ in the same manner, respectively.

Remark 2.7. Since the principal symbol of $P$ is invariant under coordinate transformations, these regions define invariant subsets in $T^{*} \widetilde{M}_{\infty}$ except the choice of the boundary decomposition. Moreover we will prove in Section 4 that these regions are also invariant under the geodesic flow generated by $p$. This property will be used to prove microlocal smoothing properties of the propagator (see Section 6).

\section{Reduction to Microlocal DisPersive estimates}

In this section we shall show that (1.5) follows from microlocal dispersive estimates. We first recall the frequency localization using the Littlewood-Paley decomposition. The following theorem was proved by Bouclet [2] for a large class of non-compact manifolds with ends (including scattering manifolds).

Proposition $3.1([2])$. Let $\psi \in C_{0}^{\infty}((0, \infty))$ be a smooth cut-off function such that

$$
\operatorname{supp} \psi \subset[1 / 4,4], \quad 0 \leq \psi \leq 1, \quad \sum_{j=0}^{\infty} \psi\left(2^{-2 j} \lambda\right)=1 \quad \text { for } \lambda \in[1, \infty) .
$$

Then, for all $\chi \in C_{0}^{\infty}(M)$ with $\operatorname{supp}(1-\chi) \subset M_{\infty}$ and $2 \leq q<\infty$ with $0 \leq d(1 / 2-1 / q) \leq 1$, there exists $C>0$ such that

$$
\|(1-\chi) u\|_{L^{q}(M)} \leq C\|u\|_{L^{2}(M)}+C\left(\sum_{j=0}^{\infty}\left\|(1-\chi) \psi\left(2^{-2 j} P\right) u\right\|_{L^{q}(M)}^{2}\right)^{\frac{1}{2}} .
$$

Using this proposition, we see that (1.5) follows from semiclassical Strichartz estimates. More precisely, it suffices to prove that for $\chi \in C_{0}^{\infty}(M)$ as above and every $\varphi \in C_{0}^{\infty}((0, \infty))$,

$$
\left\|(1-\chi) \varphi\left(h^{2} P\right) e^{-i t P} u_{0}\right\|_{L^{p}\left([0,1] ; L^{q}(M)\right)} \leq C\left\|u_{0}\right\|_{L^{2}(M)}
$$


uniformly with respect to $h \in(0,1]$, where $(p, q)$ satisfies the admissible condition (1.1). This reduction is a standard and can be proved by using the $L^{2}$-functional calculus and the almost orthogonality of $\psi\left(2^{-2 j} P\right)$. By the definition of $\widehat{P},(3.1)$ is equivalent to

$$
\left\|r^{-\frac{d-1}{2}}(1-\chi) \varphi\left(h^{2} \widehat{P}\right) e^{-i t \widehat{P}} u_{0}\right\|_{L^{p}\left([0,1] ; L^{q}(M)\right)} \leq C\left\|u_{0}\right\|_{L^{2}(\widehat{M})}, \quad h \in(0,1] .
$$

For $R>2$, we take a cut-off $\chi \in C_{0}^{\infty}(M)$ so that $\chi(z)=1$ for $r(z) \leq R, \chi(z)=0$ for $r(z) \geq R+1$. Let $J \Subset(0, \infty)$ be an open interval so that $\operatorname{supp} \varphi \Subset J$ and $N \geq d / 2$ an integer. By Lemma 2.4, we then can find a semiclassical symbol $a_{\kappa, h} \in S_{\mathrm{sc}}\left((R, \infty) \times \tilde{U}_{\kappa} \times \mathbb{R}^{d} \cap p_{\kappa}^{-1}(J)\right)$ such that $(1-\chi) \varphi\left(h^{2} \widehat{P}\right)$ is well approximated by $A_{h}:=\sum_{\kappa} \mathrm{Op}_{\kappa}^{p r}\left(a_{\kappa, h}\right)$. Moreover, the following holds.

Proposition 3.2. To prove (3.2), it suffices to show that for sufficiently large $R>1$ and all $a \in S_{\mathrm{sc}}\left((R, \infty) \times \tilde{U}_{\kappa} \times \mathbb{R}^{d} \cap p_{\kappa}^{-1}(J)\right)$,

$$
\left\|r^{-\frac{d-1}{2}} A_{h} \mathrm{Op}_{\kappa}(a) e^{-i t \widehat{P}} u_{0}\right\|_{L^{p}\left([0,1] ; L^{q}(M)\right)} \leq C\left\|u_{0}\right\|_{L^{2}(\widehat{M})}, \quad u_{0} \in C_{0}^{\infty}(M),
$$

uniformly with respect to $h \in(0,1]$, where $(p, q)$ satisfies (1.1).

This proposition is a direct consequence of Lemma 2.4, and the proof is completely same as [3, Proposition 2.18].

We next describe the spatial localization and give the main step of the proof of Theorem 1.1. The following theorem is the main result of this section. We here fix $\tilde{U}_{\kappa}$ and $J$, and hence do not write explicitly. We also use a notation such as $\Gamma^{ \pm}\left(R, \tilde{U}_{\kappa}, J, \sigma\right)=\Gamma^{ \pm}(R, \sigma)$ for short.

Theorem 3.3 (Microlocal dispersive estimates). There exist $R_{0} \geq 0$ and $\varepsilon_{0}>0$ such that the following hold for all $R_{2} \geq R_{1} \geq R_{0}, 0<\varepsilon<\varepsilon_{0}$ and $h \in(0,1]$.

(i) There exists $t_{0}>0$, independent of $R_{2}$, such that for all symbols

$$
a^{ \pm} \in S_{\mathrm{sc}}\left(\Gamma^{ \pm}\left(R_{1}, 1 / 2\right)\right), \quad b^{ \pm} \in S_{\mathrm{sc}}\left(\Omega^{ \pm}\left(R_{2}, 1 / 2\right)\right),
$$

and $0< \pm t \leq \min \left(R_{2} t_{0}, h^{-1}\right)$, we have

$$
\left\|r^{-\frac{d-1}{2}} A_{h} \mathrm{Op}_{\kappa}\left(a^{ \pm}\right) e^{-i t h \widehat{P}} \operatorname{Op}_{\kappa}\left(b^{ \pm}\right)^{*} A_{h}^{*} r^{-\frac{d-1}{2}}\right\|_{\mathcal{L}\left(L^{1}(\widehat{M}), L^{\infty}(\widehat{M})\right)} \leq C_{0}|t h|^{-\frac{d}{2}} .
$$

(ii) For all symbols

$$
a_{s}^{ \pm} \in S_{\mathrm{sc}}\left(\Gamma_{s}^{ \pm}\left(R_{1}, \varepsilon\right)\right), \quad b_{s}^{ \pm} \in S_{\mathrm{sc}}\left(\Omega_{s}^{ \pm}\left(R_{2}, \varepsilon\right)\right),
$$

and $0< \pm t \leq h^{-1}$, we have

$$
\left\|r^{-\frac{d-1}{2}} A_{h} \mathrm{Op}_{\kappa}\left(a_{s}^{ \pm}\right) e^{-i t h \widehat{P}} \mathrm{Op}_{\kappa}\left(b_{s}^{ \pm}\right)^{*} A_{h}^{*} r^{-\frac{d-1}{2}}\right\|_{\mathcal{L}\left(L^{1}(\widehat{M}), L^{\infty}(\widehat{M})\right)} \leq C_{1}|t h|^{-\frac{d}{2}} .
$$

(iii) For all $t_{1}>0$, we can find $\delta_{\varepsilon, t_{1}}>0$ and $L_{\varepsilon, t_{1}}>0$ such that for all $\sigma_{l} \in(-1,1 / 2]$ satisfying (2.12), all symbols

$$
a_{l}^{ \pm} \in S_{\mathrm{sc}}\left(\Gamma_{i}^{ \pm}\left(R_{1}, \varepsilon, \delta_{\varepsilon, t_{0}}, l\right)\right), \quad b_{l}^{ \pm} \in S_{\mathrm{sc}}\left(\Omega_{i}^{ \pm}\left(R_{2}, \varepsilon, \delta_{\varepsilon, t_{0}}, l\right)\right),
$$

and $R_{2} t_{1} \leq \pm t \leq h^{-1}$, we have

$$
\left\|r^{-\frac{d-1}{2}} A_{h} \mathrm{Op}_{\kappa}\left(a_{l}^{ \pm}\right) e^{-i t h \widehat{P}} \mathrm{Op}_{\kappa}\left(b_{l}^{ \pm}\right)^{*} A_{h}^{*} r^{-\frac{d-1}{2}}\right\|_{\mathcal{L}\left(L^{1}(\widehat{M}), L^{\infty}(\widehat{M})\right)} \leq C_{2}|t h|^{-\frac{d}{2}} .
$$

Moreover $C_{0}, C_{1}, C_{2}>0$ may be taken uniformly with respect to $h, t$ and $R_{2}$.

We give the proof of Theorem 3.3 in Section 8. Before proving Theorem 1.1, we prepare the following lemma.

Lemma 3.4. Let $R, \delta, L>0,0<\varepsilon<1$ and $\left(\sigma_{l}\right)_{1 \leq l \leq L} \subset(-1,1 / 2]$ so that $(2.12)$ is satisfied. Then for any $a \in S_{\mathrm{sc}}\left((R, \infty) \times \tilde{U}_{\kappa} \times \mathbb{R}^{d} \cap p_{\kappa}^{-1}(J)\right)$, there exist symbols $a^{ \pm} \in S_{\mathrm{sc}}\left(\Gamma^{ \pm}(R, 1 / 2)\right)$, $a_{s}^{ \pm} \in S_{\mathrm{sc}}\left(\Gamma_{s}^{ \pm}(R, \varepsilon)\right)$ and $a_{l}^{ \pm} \in S_{\mathrm{sc}}\left(\Gamma_{i}^{ \pm}(R, \varepsilon, \delta, l)\right), l=1,2, \ldots, L-1$, such that

$$
a=a^{+}+a^{-}=a_{s}^{+}+a_{s}^{-}+\sum_{l=1}^{L-1}\left(a_{l}^{+}+a_{l}^{-}\right) .
$$


Proof. Define $v(r, \theta, \rho, \omega):=\rho / \sqrt{2 p_{\kappa}(r, \theta, \rho, \omega)} . v$ is then smooth on $\Gamma^{ \pm}(R, 1 / 2)$, and satisfies

$$
\left|\partial_{r}^{j} \partial_{\theta}^{\alpha} \partial_{\rho}^{k} \partial_{\omega}^{\beta} v(r, \theta, \rho, \omega)\right| \leq C_{j \alpha k \beta} r^{-j-|\beta|} \quad \text { on } \quad \Gamma^{ \pm}(R, 1 / 2),
$$

since $p_{\kappa}$ is of the form (2.2). Take a cut-off function $\chi \in C^{\infty}(\mathbb{R})$ such that

$$
\chi(t)+\chi(-t)=1, \quad \operatorname{supp} \chi \subset(-1 / 2, \infty),
$$

and define $a^{ \pm}=\chi( \pm v) \cdot a$. Then $a^{ \pm} \in S_{\mathrm{sc}}\left(\Gamma^{ \pm}(R, 1 / 2)\right)$ and $a=a^{+}+a^{-}$. Next, choose $\chi_{s} \in C^{\infty}(\mathbb{R})$ and $\chi_{l} \in C_{0}^{\infty}(\mathbb{R}), l=1,2, \ldots, L-1$, so that

$$
\operatorname{supp} \chi_{s} \subset\left(\sqrt{1-\varepsilon^{2}}, \infty\right), \quad \operatorname{supp} \chi_{l} \subset\left(-\sigma_{l+1},-\sigma_{l-1}\right), \quad \operatorname{supp} \chi_{L} \subset\left(-1,-\sigma_{L-1}\right),
$$

and that $\chi_{s}+\sum_{l=1}^{L} \chi_{l}=1$. We define $a_{s}^{ \pm}:=\chi_{s}( \pm v) \cdot a^{ \pm}$and $a_{l}^{ \pm}:=\chi_{l}( \pm v) \cdot a^{ \pm}$, respectively. It is easy to see that they satisfy the assertion.

Proof of Theorem 1.1. Choose $R_{0}, \varepsilon_{0}, t_{0}>0$ so that (i) and (ii) in Theorem 3.3 hold for all $R_{2} \geq R_{1} \geq R_{0}$ and $0<\varepsilon \leq \varepsilon_{0}$. Next, fix $\varepsilon, \delta_{\varepsilon, t_{0}}, L_{\varepsilon, t_{0}}>0$ so that (iii) holds with $t_{1}=t_{0}$. By virtue of Proposition 3.2, it suffices to show (3.3) for any $a \in S_{\mathrm{sc}}\left(\left(R_{1}, \infty\right) \times \tilde{U}_{\kappa} \times \mathbb{R}^{d} \cap p_{\kappa}^{-1}(J)\right)$. Using Lemma 3.4 with $\delta=\delta_{\varepsilon, t_{0}}$ and $L=L_{\varepsilon, t_{0}}$, we $\operatorname{split}^{\mathrm{Op}_{\kappa}}(a)$ as follows:

$$
\mathrm{Op}_{\kappa}(a)=\mathrm{Op}_{\kappa}\left(a_{s}^{+}\right)+\mathrm{Op}_{\kappa}\left(a_{s}^{-}\right)+\sum_{l=1}^{L_{\varepsilon, t_{0}}-1}\left(\mathrm{Op}_{\kappa}\left(a_{l}^{+}\right)+\mathrm{Op}_{\kappa}\left(a_{l}^{-}\right)\right),
$$

where $a_{s}^{ \pm} \in S_{\mathrm{sc}}\left(\Gamma_{s}^{ \pm}\left(R_{1}, \varepsilon\right)\right)$ and $a_{l}^{ \pm} \in S_{\mathrm{sc}}\left(\Gamma_{i}^{ \pm}\left(R_{1}, \varepsilon, \delta_{\varepsilon, t_{0}}, l\right)\right)$. By virtue of the $T T^{*}$-argument [14], it suffices to show the $\mathcal{L}\left(L^{2}(\widehat{M}), L^{2}(M)\right)$-boundedness and $L^{1}(M)-L^{\infty}(M)$ estimates for corresponding operators uniformly with respect to $h \in(0,1]$.

By (2.6), (2.9) and the fact that $e^{-i t \widehat{P}}$ is unitary on $L^{2}(\widehat{M})$, we obtain

$$
\begin{aligned}
& \left\|r^{-\frac{d-1}{2}} A_{h} \mathrm{Op}_{\kappa}\left(a_{s}^{ \pm}\right) e^{-i t \widehat{P}} u_{0}\right\|_{L^{2}(M)} \leq C\left\|u_{0}\right\|_{L^{2}(\widehat{M})}, \\
& \left\|r^{-\frac{d-1}{2}} A_{h} \mathrm{Op}_{\kappa}\left(a_{l}^{ \pm}\right) e^{-i t \widehat{P}} u_{0}\right\|_{L^{2}(M)} \leq C\left\|u_{0}\right\|_{L^{2}(\widehat{M})},
\end{aligned}
$$

uniformly with respect to $h \in(0,1], t \in \mathbb{R}$ and $l=1,2, \ldots, L_{\varepsilon, t_{0}}-1$. After the time rescaling $t \rightarrow t h$, we set

$$
U_{s}^{ \pm}(t):=r^{-\frac{d-1}{2}} A_{h} \mathrm{Op}_{\kappa}\left(a_{s}^{ \pm}\right) e^{-i t h \widehat{P}}, \quad U_{l}^{ \pm}(t):=r^{-\frac{d-1}{2}} A_{h} \mathrm{Op}_{\kappa}\left(a_{l}^{ \pm}\right) e^{-i t h \widehat{P}} .
$$

It remains to show that $U_{s}^{ \pm}(t) U_{s}^{ \pm}(s)^{*}$ and $U_{l}^{ \pm}(t) U_{l}^{ \pm}(s)^{*}$ satisfy dispersive estimates for $0<$ $|t-s| \leq h^{-1}$. We here use a trick by [4, Lemma 4.3]. We denote by $K^{ \pm}\left(t-s, r, \theta, r^{\prime}, \theta^{\prime}\right)$ the kernel of $U_{s}^{ \pm}(t) U_{s}^{ \pm}(s)^{*}$, respectively. Since $U_{s}^{ \pm}(t) U_{s}^{ \pm}(s)^{*}=\left(U_{s}^{ \pm}(s) U_{s}^{ \pm}(t)^{*}\right)^{*}$, we see that

$$
K^{ \pm}\left(t-s, r, \theta, r^{\prime}, \theta^{\prime}\right)=\overline{K^{ \pm}\left(s-t, r^{\prime}, \theta^{\prime}, r, \theta\right)} .
$$

A same property holds for the kernel of $U_{l}^{ \pm}(t) U_{l}^{ \pm}(s)^{*}$. We hence can restrict the sign of $t-s$ so that $0 \leq \pm(t-s) \leq h^{-1}$, and it is enough to prove the following:

$$
\begin{aligned}
& \left\|U_{s}^{ \pm}(t) U_{s}^{ \pm}(s)^{*} u_{0}\right\|_{L^{\infty}(M)}+\left\|U_{l}^{ \pm}(t) U_{l}^{ \pm}(s)^{*} u_{0}\right\|_{L^{\infty}(M)} \\
& \leq C|(t-s) h|^{-\frac{d}{2}}\left\|u_{0}\right\|_{L^{1}(M)},
\end{aligned}
$$

uniformly with respect to $h \in(0,1]$ and $0< \pm(t-s) \leq h^{-1}, l=1,2, \ldots, L_{\varepsilon, t_{0}}-1$, respectively. Combining with the facts $L^{1}(M)=r^{-(d-1)} L^{1}(\widehat{M}), L^{\infty}(M)=L^{\infty}(\widehat{M})$ and

$$
\left(r^{-\frac{d-1}{2}}\right)^{*}=r^{\frac{d-1}{2}}: L^{2}(M) \rightarrow L^{2}(\widehat{M}),
$$

(3.7) follows from

$$
\begin{aligned}
& \left\|U_{s}^{ \pm}(t) V_{s}^{ \pm}(s) u_{0}||_{L^{\infty}(\widehat{M})}+\right\| U_{l}^{ \pm}(t) V_{l}^{ \pm}(s) u_{0} \|_{L^{\infty}(\widehat{M})} \\
& \leq C|(t-s) h|^{-\frac{d}{2}}\left\|u_{0}\right\|_{L^{1}(\widehat{M})},
\end{aligned}
$$


where $V_{s}^{ \pm}(s)$ and $V_{l}^{ \pm}(s)$ are given by

$$
V_{s}^{ \pm}(s):=e^{i s h \widehat{P}} \operatorname{Op}_{\kappa}\left(a_{s}^{ \pm}\right)^{*} A_{h}^{*} r^{-\frac{d-1}{2}}, \quad V_{l}^{ \pm}(s):=e^{i s h \widehat{P}} \operatorname{Op}_{\kappa}\left(a_{l}^{ \pm}\right)^{*} A_{h}^{*} r^{-\frac{d-1}{2}} .
$$

We now introduce a spatial localization. Let $\chi \in C_{0}^{\infty}((0, \infty))$ be a smooth cut-off function such that

$$
\operatorname{supp} \chi \subset[1,4], \quad 0 \leq \chi \leq 1, \quad \sum_{j=0}^{\infty} \chi\left(2^{-j} r\right)=1 \quad \text { for } r \in[2, \infty) .
$$

Choose $\chi^{\prime} \in C_{0}^{\infty}((0, \infty))$ so that $\operatorname{supp} \chi^{\prime} \subset[1 / 2,8], \chi^{\prime} \equiv 1$ on supp $\chi$, and set $\chi_{j}(r):=\chi\left(2^{-j} r\right)$, $\chi_{j}^{\prime}(r):=\chi^{\prime}\left(2^{-j} r\right)$. Since $\Psi$ is supported in a small ball centered at origin (see Definition 2.3), we obtain that

$$
\chi_{j}(r) \Psi\left(r-r^{\prime}, \theta-\theta^{\prime}\right)=\chi_{j}(r) \Psi\left(r-r^{\prime}, \theta-\theta^{\prime}\right) \chi_{j}^{\prime}\left(r^{\prime}\right), \quad j \geq j_{0},
$$

with some large $j_{0} \geq 0$. In particular, we have

$$
\mathrm{Op}_{\kappa}\left(\chi_{j} a\right)^{*} A_{h}^{*}=\mathrm{Op}_{\kappa}\left(\chi_{j} a\right)^{*} A_{h}^{*} \chi_{j}^{\prime} \quad \text { for } \quad j \geq j_{0} .
$$

If we choose $j_{0}$ as $2^{j_{0}}>R_{0}$ and set $R_{1}=2^{j_{0}}$, then

$$
a_{s}^{ \pm}=\sum_{j \geq j_{0}} \chi_{j} a_{s}^{ \pm}, \quad a_{l}^{ \pm}=\sum_{j \geq j_{0}} \chi_{j} a_{l}^{ \pm} .
$$

Since $\chi_{j} a_{s}^{ \pm} \in S_{\mathrm{sc}}\left(\Omega_{s}^{ \pm}\left(2^{j}, \varepsilon\right)\right)$ and $\chi_{j} a_{l}^{ \pm} \in S_{\mathrm{sc}}\left(\Omega_{i}^{ \pm}\left(2^{j}, \varepsilon, \delta_{\varepsilon, t_{0}}, l\right)\right)$, applying Theorem 3.3 with $R_{2}=2^{j}$ to $U_{s}^{ \pm}(t) V_{s}^{ \pm}(s)$ and $U_{l}^{ \pm}(t) V_{l}^{ \pm}(s)$, we have

$$
\begin{aligned}
& \left\|U_{s}^{ \pm}(t) V_{s}^{ \pm}(s) u_{0}\right\|_{L^{\infty}(\widehat{M})}+\left\|U_{l}^{ \pm}(t) V_{l}^{ \pm}(s) u_{0}\right\|_{L^{\infty}(\widehat{M})} \\
& \leq C|(t-s) h|^{-\frac{d}{2}} \sum_{j \geq j_{0}}\left\|\chi_{j}^{\prime} u_{0}\right\|_{L^{1}(\widehat{M})} \\
& \leq 4 C|(t-s) h|^{-\frac{d}{2}}\left\|u_{0}\right\|_{L^{1}(\widehat{M})},
\end{aligned}
$$

uniformly with respect to $h \in(0,1]$ and $0< \pm(t-s) \leq h^{-1}$. We hence obtain (3.8) and conclude the proof of Theorem 1.1.

Remark 3.5. Since $b^{ \pm}, b_{s}^{ \pm}$and $b_{l}^{ \pm}$are compactly supported with respect to both the space and the frequency, the above argument tells us that Strichartz estimates follows from microlocal dispersive estimates.

\section{Classical Trajectories}

In this section we study the behavior of the geodesic flow which we denote by

$$
\exp t H_{p}: T^{*} M \rightarrow T^{*} M
$$

Recall that the principal symbol $p_{\kappa}$ of $\widehat{P}_{\kappa}$ is of the form

$$
p_{\kappa}(r, \theta, \rho, \omega)=\frac{1}{2} \rho^{2}+\frac{1}{2 r^{2}}\left(h^{j k}(\theta)+a^{j k}(r, \theta)\right) \omega_{j} \omega_{k}, \quad(r, \theta, \rho, \omega) \in(1, \infty) \times U_{\kappa} \times \mathbb{R}^{d},
$$

where $a^{j k}(r, \theta)$ satisfies (2.1). We put

$$
(r(t), \theta(t), \rho(t), \omega(t))=\exp t H_{p_{\kappa}}(r, \theta, \rho, \omega),
$$


which solves the following Hamilton equation:

$$
\left\{\begin{aligned}
\dot{r}(t)= & \rho(t), \\
\dot{\theta}^{j}(t)= & \frac{1}{r(t)^{2}} h^{j k}(\theta(t)) \omega_{k}(t)+\frac{1}{r(t)^{2}} a^{j k}(r(t), \theta(t)) \omega_{k}, \\
\dot{\rho}(t)= & \frac{1}{r(t)^{3}} h^{j k}(\theta(t)) \omega_{j}(t) \omega_{k}(t)+\frac{1}{r(t)^{3}} a^{j k}(r(t), \theta(t)) \omega_{j}(t) \omega_{k}(t) \\
& -\frac{1}{2 r^{2}} \frac{\partial a^{j k}}{\partial r}(r(t), \theta(t)) \omega_{j}(t) \omega_{k}(t), \\
\dot{\omega}_{j}(t)= & -\frac{1}{2 r(t)^{2}} \frac{\partial h^{k l}}{\partial \theta^{j}}(\theta(t)) \omega_{k}(t) \omega_{l}(t)-\frac{1}{2 r(t)^{2}} \frac{\partial a^{k l}}{\partial \theta^{j}}(r(t), \theta(t)) \omega_{k}(t) \omega_{l}(t) .
\end{aligned}\right.
$$

We first prepare an a priori estimate for $\exp t H_{p_{\kappa}}$.

Lemma 4.1. Let $J \Subset(0, \infty)$ and $-1<\sigma<1$. Then, there exist $R_{0}>0$ such that for all $R \geq R_{0}$,

$$
C^{-1}(r+|t|) \leq r(t) \leq C(r+|t|), \quad|\rho(t)|+|\omega(t) / r| \leq C,
$$

uniformly with respect to $(r, \theta, \rho, \omega) \in \Gamma^{ \pm}\left(R, \tilde{U}_{\kappa}, J, \sigma\right)$ and $\pm t \geq 0$, where the constant $C$ may be taken uniformly with respect to $R$ and $t$.

Using Lemma 4.1, we obtain the behavior of the geodesic flow near infinity.

Proposition 4.2. Let $R_{0}>0$ be as in Lemma 4.1 and $R \geq R_{0}$. Then the following estimates hold for all $(r, \theta, \rho, \omega) \in \Gamma^{ \pm}\left(R, \tilde{U}_{\kappa}, J, \sigma\right), \pm t \geq 0$ and $(j, \alpha, k, \beta) \in \mathbb{Z}_{+}^{2 d}$ as long as the trajectory belongs to the same coordinate neighborhood $(1, \infty) \times U_{\kappa}$ :

$$
\begin{gathered}
\left\{\begin{aligned}
\left|\partial_{r}^{j} \partial_{\theta}^{\alpha} \partial_{\rho}^{k} \partial_{\omega}^{\beta}(r(t)-r \mp t \rho)\right| & \leq C r^{-j-|\beta|}|\omega / r|^{(2-|\beta|)+}\langle r / t\rangle^{-1}|t|, \\
\left|\partial_{r}^{j} \partial_{\theta}^{\alpha} \partial_{\rho}^{k} \partial_{\omega}^{\beta}(\theta(t)-\theta)\right| & \leq C r^{-j-|\beta|}|\omega / r|^{(1-|\beta|)+}\langle r / t\rangle^{-1}, \\
\left|\partial_{r}^{j} \partial_{\theta}^{\alpha} \partial_{\rho}^{k} \partial_{\omega}^{\beta}(\rho(t)-\rho)\right| & \leq C r^{-j-|\beta|}|\omega / r|^{(2-|\beta|)_{+}}\langle r / t\rangle^{-1}, \\
\left|\partial_{r}^{j} \partial_{\theta}^{\alpha} \partial_{\rho}^{k} \partial_{\omega}^{\beta}(\omega(t)-\omega)\right| & \leq C r^{1-j-|\beta|}|\omega / r|^{(2-|\beta|)_{+}}\langle r / t\rangle^{-1},
\end{aligned}\right. \\
\left\{\begin{aligned}
\left|\partial_{r}^{j} \partial_{\theta}^{\alpha} \partial_{\rho}^{k} \partial_{\omega}^{\beta}(r(t)-r)\right|+\left|\partial_{r}^{j} \partial_{\theta}^{\alpha} \partial_{\rho}^{k} \partial_{\omega}^{\beta}(\omega(t)-\omega)\right| \leq C r^{-j-|\beta|}|t|, \\
\left|\partial_{r}^{j} \partial_{\theta}^{\alpha} \partial_{\rho}^{k} \partial_{\omega}^{\beta}(\theta(t)-\theta)\right|+\left|\partial_{r}^{j} \partial_{\theta}^{\alpha} \partial_{\rho}^{k} \partial_{\omega}^{\beta}(\rho(t)-\rho)\right| \leq C r^{-1-j-|\beta|}|t|,
\end{aligned}\right.
\end{gathered}
$$

Moreover, for all $(r, \theta, \rho, \omega) \in \Gamma^{ \pm}\left(R, \tilde{U}_{\kappa}, J, \sigma\right)$ and $\pm t \geq 0$, we have

$$
\left|\sqrt{2 E_{0}} \mp \rho(t)\right| \leq C|\omega / r|^{2}\langle t / r\rangle^{-1},
$$

where $E_{0}:=p_{\kappa}(r, \theta, \rho, \omega)$ is the initial energy.

Proof of Lemma 4.1. We prove the lemma for $t \geq 0$, and the proof for $t \leq 0$ is analogous. We first note that the energy conservation low, namely

$$
E_{0}=p_{\kappa}(r(t), \theta(t), \rho(t), \omega(t)), \quad t \in \mathbb{R},
$$

implies $|\rho(t)|+|\omega(t) / r(t)| \leq C_{0}$ as long as $r(t)$ large enough, where $C_{0}>0$ depends only on $\sqrt{E_{0}}$. In particular, we can find $R_{1}, C_{1}>0$ so large that

$$
\dot{r}(0)=O(1), \dot{\rho}(0)=\frac{1}{r^{3}} h^{j k}(\theta) \omega_{j} \omega_{k}+O\left(r^{-1-\mu}\right),
$$

for $r>R_{1}$, and hence

$$
\left.\frac{d^{2}}{d t^{2}} r(t)^{2}\right|_{t=0}=\left.2(\dot{r}(t) \rho(t)+r(t) \dot{\rho}(t))\right|_{t=0} \geq 4 E_{0}-C_{1} r^{-\mu} .
$$

Since $\sigma \in(-1,1)$, we can choose $0<\delta<1$ and $C_{2}>0$ so that

$$
r^{2}-2 \sigma r \sqrt{2 E_{0}} t+2(1-\delta)^{2} E_{0} t^{2} \geq \frac{1}{C_{2}^{2}}\left(r+t \sqrt{2 E_{0}}\right)^{2}, r \geq 0, t \geq 0 .
$$


We now suppose that there exist $R>0$ and $t_{0}>0$ such that

$$
4 E_{0}-C_{1} r(t)^{-\mu} \geq 4(1-\delta)^{2} E_{0}
$$

holds true for $r>R$ and $0 \leq t \leq t_{0}$. We then have

$$
\begin{aligned}
r(t)^{2} & \geq r^{2}+2 r \rho t+2(1-\delta)^{2} E_{0} t^{2} \\
& \geq r^{2}-2 \sigma r \sqrt{2 E_{0}} t+2(1-\delta)^{2} E_{0} t^{2} \\
& \geq \frac{1}{C_{2}^{2}}\left(r+t \sqrt{2 E_{0}}\right)^{2},
\end{aligned}
$$

for $0 \leq t \leq t_{0}$ and $(r, \theta, \rho, \omega) \in \Gamma^{+}\left(R, \tilde{U}_{\kappa}, J, \sigma\right)$. Moreover, if we put $q_{0}(t)=h^{j k}(\theta(t)) \omega_{j}(t) \omega_{k}(t)$, then a direct computation yields

$$
\begin{aligned}
\dot{q}_{0}(t) & =\dot{\theta}^{l} \frac{\partial h^{j k}}{\partial \theta^{l}} \omega_{j} \omega_{k}+2 h^{j k} \dot{\omega}_{j} \omega_{k} \\
& =\frac{1}{r^{2}}\left(h^{l m}+a^{l m}\right) \omega_{m} \frac{\partial h^{j k}}{\partial \theta^{l}} \omega_{j} \omega_{k}-h^{j k} \omega_{k} \frac{1}{r^{2}}\left(\frac{\partial h^{l m}}{\partial \theta^{j}}+\frac{\partial a^{l m}}{\partial \theta^{j}} \omega_{l} \omega_{m}\right) \\
& =O\left(r(t)^{-1-\mu} q_{0}(t)\right) .
\end{aligned}
$$

Integrating with respect to $s \in[0, t]$, we have

$$
q_{0}(t) \leq q_{0}(0)+C \int_{0}^{t}\left(r+|s| \sqrt{2 E_{0}}\right)^{-1-\mu} q_{0}(s) d s, \quad 0 \leq t \leq t_{0} .
$$

By Gronwall's inequality and the ellipticity of $q_{0}$, we obtain

$$
|\omega(t)| \leq C_{3} \sqrt{q_{0}} \leq C_{3} r \sqrt{2 E_{0}} \text { for } 0 \leq t \leq t_{0} .
$$

Applying (4.7) to (4.1), we have

$$
\dot{r}(t)=\rho(t), \dot{\theta}(t)=O\left(r(t)^{-2} r\right), \dot{\rho}(t)=O\left(r(t)^{-3} r^{2}\right), \dot{\omega}(t)=O\left(r(t)^{-2} r^{2}\right) .
$$

In particular, we see that $|\rho(t)| \leq C_{4} \sqrt{2 E_{0}}$ for $0 \leq t \leq t_{0}$ with some large $C_{4}>0$. Therefore, it is enough to check that (4.6) holds with $t_{0}=\infty$. Define

$$
S:=\{t \geq 0 \mid(4.6) \text { holds for all } s \in[0, t]\} .
$$

For sufficiently large $r>R_{1}$, the above argument shows that $0 \in S$ and $S \neq \emptyset$. Set $t_{0}=\sup S$. The above argument then implies

$$
r(t) \geq C_{2}^{-1}\left(r+t \sqrt{2 E_{0}}\right) \quad \text { for } \quad r>R_{2}, 0 \leq t \leq t_{0},
$$

with some $R_{2}>R_{1}$ large enough. Taking $R_{3}>R_{2}$ so that

$$
4 \delta E_{0}-\delta^{2} E_{0} \geq C_{1} C_{2}^{\mu} r^{-\mu} \text { for } r>R_{3},
$$

we have

$$
\begin{aligned}
4 E_{0}-C_{1} r(t)^{-\mu} & \geq 4\left(1-\frac{\delta}{2}\right)^{2} E_{0}+4 \delta E_{0}-\delta^{2} E_{0}-C_{1} C_{2}^{\mu}\left(r+t \sqrt{2 E_{0}}\right)^{-\mu} \\
& \geq 4\left(1-\frac{\delta}{2}\right)^{2} E_{0}
\end{aligned}
$$

for $r>R_{3}$ and $0 \leq t \leq t_{0}$. Therefore, $t_{0}+\varepsilon \in S$ for some $\varepsilon>0$ which implies $t_{0}=\infty$ by the definition of $t_{0}$. The estimate of $r(t)$ from above is obvious.

Proof of Proposition 4.2. Let $t \geq 0$. The proof for $t<0$ is similar. Take $R_{0}>0$ as in Lemma 4.1. (4.3) with $j+|\alpha|+k+|\beta|=0$ is a direct consequence of Lemma 4.1 and (4.8) since

$$
\int_{0}^{t}(r+|s|)^{-1-a} d s \leq C r^{-a}\langle r / t\rangle^{-1} \text { for any } a>0 .
$$

We next consider the derivatives. Put $z(t):=r(t)-t \rho(t)$. It is easy to see that

$$
W(t):=(z(t), \theta(t), \rho(t), \omega(t))=\exp \left(-t H_{\frac{1}{2} \rho^{2}}\right) \circ \exp t H_{p_{\kappa}}(r, \theta, \rho, \omega)
$$


solves the following Hamilton equations:

$$
\dot{z}=\frac{\partial K}{\partial \rho}, \dot{\theta}=\frac{\partial K}{\partial \omega}, \dot{\rho}=-\frac{\partial K}{\partial z}, \dot{\omega}=-\frac{\partial K}{\partial \theta},
$$

with a time dependent Hamiltonian:

$$
K(t, z, \theta, \rho, \omega):=\frac{1}{2} \rho^{2}-p_{\kappa}(z+t \rho, \theta, \rho, \omega)=-\frac{h^{j k}(\theta)+a_{3}^{j k}(z+t \rho, \theta)}{2(z+t \rho)^{2}} \omega_{j} \omega_{k} .
$$

Lemma 4.1 shows that $K(t, W(t))$ satisfies

$$
\left|\left(\partial_{z}^{j} \partial_{\theta}^{\alpha} \partial_{\rho}^{k} \partial_{\omega}^{\beta} K\right)(t, W(t))\right| \leq C r(t)^{-2-j}|\omega|^{(2-|\beta|)+} \quad \text { if }|\beta| \leq 2,
$$

and $\left(\partial_{z}^{j} \partial_{\theta}^{\alpha} \partial_{\rho}^{k} \partial_{\omega}^{\beta} K\right)(t, W(t))=0$ if $|\beta| \geq 3$. Let $\gamma=(j, \alpha, k, \beta),|\gamma|=1$, and denote $\partial^{\gamma}=$ $\partial_{r}^{j} \partial_{\theta}^{\alpha} \partial_{\rho}^{k} \partial_{\omega}^{\beta}$ for short. By differentiating the Hamilton equation with respect to $\partial^{\gamma}$, we have

$$
\partial_{t} \partial^{\gamma}(W(t)-W(0))=A(t) \partial^{\gamma}(W(t)-W(0))+A(t) \partial^{\gamma} W(0),
$$

where $A(t)=\left(A_{j}(t)\right)_{1 \leq j \leq 4}:=d H_{K}(W(t))$ satisfies

$$
\begin{aligned}
& \left|A_{1}(t)\right| \leq C r(t)^{-2}|\omega|^{2}, \quad\left|A_{2}(t)\right| \leq C r(t)^{-2}|\omega|, \\
& \left|A_{3}(t)\right| \leq C r(t)^{-3}|\omega|^{2}, \quad\left|A_{4}(t)\right| \leq C r(t)^{-2}|\omega|^{2} .
\end{aligned}
$$

If we put

$$
\begin{aligned}
f(t) & =\left(f_{1}(t), f_{2}(t), f_{3}(t), f_{4}(t)\right) \\
& :=\left(\frac{\partial^{\gamma}(z(t)-r)}{r}, \partial^{\gamma}(\theta(t)-\theta), \partial^{\gamma}(\rho(t)-\rho), \frac{\partial^{\gamma}(\omega(t)-\omega)}{r}\right),
\end{aligned}
$$

then, by using (4.2), (4.9), (4.10) and (4.11), we have

$$
\left|f_{1}(t)\right| \leq C \int_{0}^{t}\left(r^{-1} r(s)^{-2-j}|\omega|^{(2-|\beta|)_{+}}+r(s)^{-2} r|f(s)|\right) d s .
$$

We similarly obtain

$$
\begin{aligned}
& \left|f_{2}(t)\right| \leq C \int_{0}^{t}\left(r(s)^{-2-j}|\omega|^{(1-|\beta|)_{+}}+r(s)^{-2} r|f(s)|\right) d s \\
& \left|f_{3}(t)\right| \leq C \int_{0}^{t}\left(r^{-1} r(s)^{-2-j}|\omega|^{(2-|\beta|)_{+}}+r(s)^{-2} r|f(s)|\right) d s \\
& \left|f_{4}(t)\right| \leq C \int_{0}^{t}\left(r^{-1} r(s)^{-2-j}|\omega|^{(2-|\beta|)_{+}}+r(s)^{-2} r|f(s)|\right) d s .
\end{aligned}
$$

Gronwall's inequality then implies

$$
|f(t)| \leq C \int_{0}^{t} r(s)^{-2-j}|\omega|^{(1-|\beta|)+} d s \lesssim r^{-1-j}|\omega|^{(1-|\beta|)+}\langle r / t\rangle^{-1}
$$

and we obtain the estimate for $\partial^{\gamma}(\theta(t)-\theta)$. For the proof on other variables, we set

$$
g(t):=\left(r^{-1} \partial^{\gamma}(z(t)-r), \partial^{\gamma}(\rho(t)-\rho), r^{-1} \partial^{\gamma}(\omega(t)-\omega) .\right.
$$

Combining the second estimate of (4.3) with (4.9), (4.12), (4.14) and (4.15), we have

$$
|g(t)| \lesssim \int_{0}^{t}\left(r^{-1-j} r(s)^{-2}|\omega|^{(2-|\beta|)+}+r(s)^{-2} r|g(s)|\right) d s .
$$

Again Gronwall's inequality implies

$$
|g(t)| \leq C r^{-2-j}|\omega|^{(2-|\beta|)+}\langle r / t\rangle^{-1}
$$

and we obtain the estimates for $\partial^{\gamma} \rho(t), \partial^{\gamma} \omega(t)$. Moreover the first estimate of (4.3) follows from

$$
\partial^{\gamma}(\dot{r}(t)-\rho)=\partial^{\gamma}(\rho(t)-\rho)=O\left(r^{-2-j}|\omega|^{(2-|\beta|)+}\langle r / t\rangle^{-1}\right),
$$


since $\langle r / t\rangle^{-1}$ is monotone increasing with respect to $t$. Next, let $l$ be a non-negative integer and suppose that (4.13) holds for any $\gamma$ with $|\gamma| \leq l$. Let $\gamma=(j, \alpha, k, \beta),|\gamma|=l+1$. A direct computation yields

$$
\partial_{t} \partial^{\gamma}(W(t)-W(0))=A(t) \partial^{\gamma}(W(t)-W(0))+A(t) \partial^{\gamma} W(0)+B(t),
$$

where $B(t)$ is a linear combination of products of

$$
\begin{aligned}
& \left(\partial_{z}^{\alpha_{1}} \partial_{\theta}^{\alpha^{\prime}} \partial_{\rho}^{\alpha_{d+1}} \partial_{\omega}^{\alpha^{\prime \prime}} d H_{K}\right)(W(t)),
\end{aligned}
$$

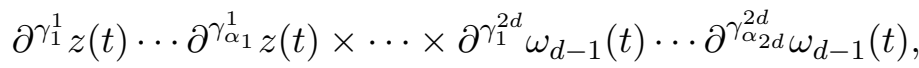

with $\alpha, \gamma_{1}^{1}, \gamma_{2}^{1}, \ldots, \gamma_{\alpha_{2 d}}^{2 d} \in \mathbb{Z}_{+}^{2 d}$ such that

$$
\begin{gathered}
\alpha=\left(\alpha_{1}, \ldots, \alpha_{2 d}\right)=\left(\alpha_{1}, \alpha^{\prime}, \alpha_{d+1}, \alpha^{\prime \prime}\right) \in \mathbb{Z}_{+}^{1+(d-1)+1+(d-1)}, \quad 1 \leq|\alpha| \leq|\gamma|, \\
\gamma_{1}^{1}+\gamma_{2}^{1}+\cdots+\gamma_{\alpha_{2 d}}^{2 d}=\gamma, \quad 1 \leq \gamma_{l}^{k} \leq|\gamma|-1 .
\end{gathered}
$$

The induction hypothesis implies that each entry of $B(t)=\left(B_{j}(t)\right)$ satisfies

$$
\begin{aligned}
& \left|B_{1}(t)\right|+\left|B_{4}(t)\right| \\
& \leq C \sum_{\substack{1 \leq|\alpha| \leq|\gamma|,\left|\alpha^{\prime \prime}\right| \leq 2}} r(t)^{-2-\alpha_{1}} r^{2-\left|\alpha^{\prime \prime}\right|}|\nu|^{2-\left|\alpha^{\prime \prime}\right|} r^{\alpha_{1}+\left|\alpha^{\prime \prime}\right|} r^{-j-|\beta|}|\nu|^{\left(2|\alpha|-\left|\alpha^{\prime}\right|-\left|\alpha^{\prime \prime}\right|-|\beta|\right)_{+}} \\
& \leq C r(t)^{-2} r^{-j-|\beta|}|\nu|^{(2-|\beta|)_{+}},
\end{aligned}
$$

and we similarly obtain

$$
\left|B_{2}(t)\right| \leq C r(t)^{-2} r^{-j-|\beta|}|\nu|^{(1-|\beta|)_{+}}, \quad\left|B_{3}(t)\right| \leq C r(t)^{-3} r^{-j-|\beta|}|\nu|^{(2-|\beta|)_{+}},
$$

where $\nu=\omega / r$. By a similar argument as that in the case for $|\gamma|=1$, we obtain the assertion. The proof of (4.4) is more simpler than (4.3), and we hence omit it.

Finally, we prove (4.5). Note that if $R>0$ is large enough, then

$$
\dot{\rho}(t)=r(t)^{-3}\left(h^{j k}(\theta(t))+O\left(r(t)^{-\mu}\right)\right) \omega_{j}(t) \omega_{k}(t) \geq 0, \quad t \in \mathbb{R} .
$$

Therefore, integrating $\dot{\rho}(t)$ with respect to $t$, we have (4.5).

Proposition 4.2 implies that the trajectory belongs to a fixed coordinate neighborhood as long as either $(r, \theta, \rho, \omega) \in \Gamma^{ \pm}\left(R, \tilde{U}_{\kappa}, J, \sigma\right)$ and $0 \leq \pm t \leq r t_{0}$ or $(r, \theta, \rho, \omega) \in \Gamma_{s}^{ \pm}\left(R, \tilde{U}_{\kappa}, J, \varepsilon_{0}\right)$ and $\pm t \geq 0$, respectively, provided $t_{0}, \varepsilon_{0}>0$ are small enough. It also follows from Proposition 4.2 that the outgoing and incoming regions are invariant under the geodesic flow (except the choice of the boundary decomposition). More precisely we have the following.

Corollary 4.3. Let $R_{0}>0$ be as in Lemma 4.1. Fix $U_{\kappa}, \tilde{U}_{\kappa}, U_{\kappa^{\prime}}, \tilde{U}_{\kappa^{\prime}}$ and $\kappa^{\prime}: V_{\kappa^{\prime}} \rightarrow U_{\kappa^{\prime}}$ so that $\tilde{U}_{\kappa} \Subset U_{\kappa}$ and $\tilde{U}_{\kappa^{\prime}} \Subset U_{\kappa^{\prime}}$. Then there exists $C>0$ such that, for all $R \geq R_{0}, T_{0}>0$ and $(r, \theta, \rho, \omega) \in \Gamma^{+}\left(R, \tilde{U}_{\kappa}, J, \sigma\right)$, we have

$$
\kappa_{*}^{\prime} \exp T_{0} H_{p} \kappa_{*}^{-1}(r, \theta, \rho, \omega) \in \Gamma^{+}\left(\left(R+T_{0}\right) / C, \tilde{U}_{\kappa^{\prime}}, J, \sigma\right) .
$$

In particular we can find $t_{0}>0$ such that, for all $(r, \theta, \rho, \omega) \in \Gamma^{+}\left(R, \tilde{U}_{\kappa}, J, \sigma\right)$ and $0 \leq t \leq r t_{0}$,

$$
\exp t H_{p_{\kappa}}(r, \theta, \rho, \omega) \in \Gamma^{+}\left((R+t) / C, \tilde{U}_{\kappa, \sqrt{t_{0}}}, J, \sigma\right),
$$

where $\tilde{U}_{\kappa, \sqrt{t_{0}}}$ is the $\sqrt{t_{0}}$-neighborhood of $\tilde{U}_{\kappa}$. Moreover there exists a small constant $\varepsilon_{0}>0$ such that if $0<\varepsilon \leq \varepsilon_{0},(r, \theta, \rho, \omega) \in \Gamma_{s}^{+}\left(R, \tilde{U}_{\kappa}, J, \varepsilon\right)$ and $t \geq 0$, then

$$
\exp t H_{p_{\kappa}}(r, \theta, \rho, \omega) \in \Gamma_{s}^{+}\left(R / C, \tilde{U}_{\kappa}, J, \varepsilon\right) .
$$

When $t<0$, analogous results hold in the incoming region. 
Proof. We first prove (4.18) and (4.19). By Lemma 4.1, we can find $R>0$ large enough such that

$$
\dot{\rho}(t)=\frac{1}{r(t)^{3}}\left(h^{j k}(\theta(t))-O\left(r(t)^{-\mu}\right) \omega_{j}(t) \omega_{k}(t) \geq 0, \quad r>R, t \geq 0,\right.
$$

and hence $\rho(t) \geq \rho$ for $t \geq 0$. Therefore, (4.18) follows from (4.2), (4.4) and the energy conservation $p_{\kappa}(r(t), \theta(t), \rho(t), \omega(t))=p_{\kappa}(r, \theta, \rho, \omega)$. Next, let $(r, \theta, \rho, \omega) \in \Gamma_{s}^{+}\left(R, \tilde{U}_{\kappa}, J, \varepsilon\right)$. Since $\langle r / t\rangle^{-1} \leq 1$ and

$$
|\omega / r| \leq C \varepsilon \quad \text { on } \quad \Gamma_{s}^{ \pm}\left(R, \tilde{U}_{\kappa}, J, \varepsilon\right)
$$

(4.19) follows from (4.3). To prove (4.17), divide the time interval $\left[0, T_{0}\right]$ as

$$
\left[0, T_{0}\right] \subset\left[0, R t_{0}\right] \cup\left[R t_{0}, 2 R t_{0}\right] \cup \cdots \cup\left[T_{0}-R t_{0}, T_{0}\right] .
$$

In each interval $\left[j R t_{0},(j+1) R t_{0}\right]$, the flow is contained some fixed coordinate neighborhood. Since the outgoing region is invariant under coordinate transformations, applying (4.18) on each chart, we have the assertion.

\section{The IsOzaki-Kitada PARAmetrix}

In this section we construct the Isozaki-Kitada parametrix of $e^{-i t h P} \mathrm{Op}_{\kappa}\left(a_{s}^{ \pm}\right)$, where the symbols $a_{s}^{+}$and $a_{s}^{-}$are supported in the strongly outgoing incoming regions, respectively. Though the method of construction is similar to the case of an asymptotically hyperbolic manifold, the class of the phase function of the parametrix becomes even worse (see Remark 5.2). We thus give the full details of the proof.

By Corollary 4.3, we can always work on one fixed coordinate chart $\left(U_{\kappa}, \kappa\right)$, and hence drop the subscript $\kappa$ if there is no confusion. Fix an open set $\tilde{U}_{\kappa} \Subset U_{\kappa}$ with $\tilde{\Psi}_{\kappa}=1$ on $(2, \infty) \times \tilde{U}_{\kappa}$ and an open interval $J \Subset(0, \infty)$ arbitrarily. We denote $\Gamma^{ \pm}(R, \varepsilon)=\Gamma_{s}^{ \pm}\left(R, \tilde{U}_{\kappa}, J, \varepsilon\right)$ for short. For a large parameter $\lambda \geq 1$, we also denote $\Gamma_{s}^{ \pm}(\lambda)=\Gamma_{s}^{ \pm}\left(R / \lambda, \tilde{U}_{\kappa}, J, \lambda \varepsilon\right)$. Notice that $\Gamma_{s}^{ \pm}(\lambda)$ and $\Omega_{s}^{ \pm}(\lambda)$ is increasing with respect to $\lambda: \Gamma_{s}^{ \pm}(R, \varepsilon) \subset \Gamma_{s}^{ \pm}\left(\lambda_{1}\right) \subset \Gamma_{s}^{ \pm}\left(\lambda_{2}\right), 1 \leq \lambda_{1}<\lambda_{2}$.

5.1. Fourier integral operators for the Isozaki-Kitada parametrix. We here study Fourier integral operators (FIO's for short) on $\mathbb{R}^{d}$ which will be used to construct the Isozaki-Kitada parametrix. The first step is to construct the corresponding phase function.

Theorem 5.1. There exist $R_{0}, \lambda_{0}>0$ large enough and $\varepsilon_{0}>0$ small enough such that for all $R, \varepsilon, \lambda>0$ satisfying $\lambda \geq \lambda_{0}, R \geq \lambda R_{0}$ and $0<\varepsilon \leq \varepsilon_{0} / \lambda$, we can find smooth and real-valued functions $S^{ \pm} \in C^{\infty}\left(\mathbb{R}^{2 d}, \mathbb{R}\right)$ satisfying the Eikonal equation:

$$
p\left(r, \theta, \partial_{r} S^{ \pm}(r, \theta, \rho, \omega), \partial_{\theta} S^{ \pm}(r, \theta, \rho, \omega)\right)=\frac{1}{2} \rho^{2}, \quad(r, \theta, \rho, \omega) \in \Gamma_{s}^{ \pm}(R, \varepsilon) .
$$

If we put $\varphi^{ \pm}(r, \theta, \rho, \omega):=S^{ \pm}(r, \theta, \rho, \omega)-r \rho-\theta \cdot \omega$, then $\varphi^{ \pm}$satisfy $\operatorname{supp} \varphi^{ \pm} \subset \Gamma_{s}^{ \pm}(\lambda)$, and

$$
\left|\partial_{r}^{j} \partial_{\theta}^{\alpha} \partial_{\rho}^{k} \partial_{\omega}^{\beta} \varphi^{ \pm}(r, \theta, \rho, \omega)\right| \leq C r^{1-j-|\beta|}|\omega / r|^{(2-|\beta|)_{+}} \quad \text { on } \quad \Gamma_{s}^{ \pm}(\lambda) .
$$

Furthermore, we can write

$$
\varphi^{ \pm}(r, \theta, \rho, \omega)=\frac{1}{2 r \rho} q_{0}(\theta, \omega)+R^{ \pm}(r, \theta, \rho, \omega) \quad \text { on } \quad \Gamma_{s}^{ \pm}(R, \varepsilon),
$$

where $q_{0}(\theta, \omega):=h^{j k}(\theta) \omega_{j} \omega_{k}$ and $R^{ \pm}(r, \theta, \rho, \omega)$ satisfy

$$
\left|\partial_{r}^{j} \partial_{\theta}^{\alpha} \partial_{\rho}^{k} \partial_{\omega}^{\beta} R^{ \pm}(r, \theta, \rho, \omega)\right| \leq C r^{1-j-|\beta|}\left(|\omega / r|^{(3-|\beta|)_{+}}+r^{-\mu}|\omega / r|^{(2-|\beta|)_{+}}\right) .
$$

Here the constant $C>0$ can be taken uniformly with respect to $R, \varepsilon$ and $\lambda$.

Remark 5.2. We remark that $\varphi^{ \pm}$and its derivatives with respect to $(\theta, \rho)$ are not bounded with respect to $r$ even for the perfectly conic $\left(a_{j k} \equiv 0\right)$ case. This condition is even worse than that of the asymptotically flat case or asymptotically hyperbolic case. Indeed, we see that $\partial_{x}^{\alpha} \partial_{\xi}^{\beta}\left(S^{ \pm}(x, \xi)-x \cdot \xi\right)=O\left(\langle x\rangle^{1-\varepsilon-|\alpha|}\right)$ with some $\varepsilon>0$ in the asymptotically flat case [4], and $\partial_{r}^{j} \partial_{\theta}^{\alpha} \partial_{\rho}^{k} \partial_{\omega}^{\beta}\left(S^{ \pm}(r, \theta, \rho, \omega)-r \rho-\theta \cdot \omega\right)=O\left(e^{-r|\beta|}\right)$ in the asymptotically hyperbolic case [3]. We 
refer to $[4,3]$ for more details. We also refer to the original paper by Isozaki-Kitada [12] which was concerned with a long-range potential scattering theory on $\mathbb{R}^{d}$.

We also note that (5.1) implies

$$
\left|\partial_{r}^{j} \partial_{\theta}^{\alpha} \partial_{\rho}^{k} \partial_{\omega}^{\beta} \varphi^{ \pm}(r, \theta, \rho, \omega)\right| \leq C\langle r\rangle^{1-j-|\beta|} \varepsilon_{0}^{(2-|\beta|)_{+}} \text {on } \quad \mathbb{R}^{2 d},
$$

since $|\omega / r| \leq C \lambda \varepsilon \leq C \varepsilon_{0}$ on $\operatorname{supp} \varphi^{ \pm}\left(\subset \Gamma_{s}^{ \pm}(\lambda)\right)$. For sufficiently large $R_{0}>0$ and sufficiently small $\varepsilon_{0}>0$, we hence have

$$
1 / 2<\left|\operatorname{det}^{t} \partial_{\rho, \omega} \partial_{r, \theta} S^{ \pm}(r, \theta, \rho, \omega)\right|<3 / 2 \quad \text { on } \quad \mathbb{R}^{2 d},
$$

though $\left|{ }^{t} \partial_{\rho, \omega} \partial_{r, \theta} S^{ \pm}-\mathrm{Id}\right|$ is not bounded with respect to $r$ in general. This estimate is crucial to obtain $L^{2}$-boundedness of FIO's.

To prove Theorem 5.1, we prepare several lemmas.

Lemma 5.3. There exist $R_{0}>0$ large enough and $\varepsilon_{0}>0$ small enough such that, for all $R, \varepsilon>0, \lambda_{0} \geq 1$ satisfying $R \geq \lambda_{0} R_{0}, \varepsilon \leq \varepsilon_{0} / \lambda_{0}$ and all $\pm t \geq 0$, the maps

$$
f^{ \pm}(t):(r, \theta, \rho, \omega) \mapsto(r, \theta, \rho(t, r, \theta, \rho, \omega), \omega(t, r, \theta, \rho, \omega))
$$

are diffeomorphisms from $\Gamma_{s}^{ \pm}\left(\lambda_{0}\right)$ onto its range, respectively. Moreover, for sufficiently large $\lambda_{0}>0$, we have

$$
\Gamma_{s}^{ \pm}(R, \varepsilon) \subset f^{ \pm}(t)\left(\Gamma_{s}^{ \pm}\left(\lambda_{0}\right)\right), \quad \pm t \geq 0 .
$$

Proof. We prove the lemma for $t \geq 0$ only, and the proof for $t \leq 0$ is similar. Let $F$ : $(r, \theta, \rho, \omega) \mapsto(r, \theta, \rho, \omega / r)$ be a global diffeomorphism from $(0, \infty) \times \mathbb{R}^{d-1}$ onto itself, and we define for $(r, \theta, \rho, \nu) \in F \Gamma_{s}^{+}\left(\lambda_{0}\right)$,

$$
\tilde{f}^{+}(t)(r, \theta, \rho, \nu)=(r, \theta, \tilde{\rho}(t), \tilde{\omega}(t)):=\left(F \circ f^{+}(t) \circ F^{-1}\right)(r, \theta, \rho, \nu),
$$

where $\nu=\omega / r$. By (4.3), we can choose $R_{0}, \varepsilon_{0}>0$ and $C_{0}>0$ such that

$$
\begin{gathered}
\left|\partial_{r}^{j} \partial_{\theta}^{\alpha} \partial_{\rho}^{k} \partial_{\nu}^{\beta}(\tilde{\rho}(t)-\rho)\right| \leq C_{0} r^{-j}|\nu|^{(2-|\beta|)_{+}} \leq C_{0} R_{0}^{-j} \varepsilon_{0}^{(2-|\beta|)_{+}}, \\
\left|\partial_{r}^{j} \partial_{\theta}^{\alpha} \partial_{\rho}^{k} \partial_{\nu}^{\beta}(\tilde{\omega}(t)-\nu)\right| \leq C_{0} r^{-j}|\nu|^{(2-|\beta|)_{+}} \leq C_{0} R_{0}^{-j} \varepsilon_{0}^{(2-|\beta|)_{+}},
\end{gathered}
$$

and hence

$$
\left|\partial_{r}^{j} \partial_{\theta}^{\alpha} \partial_{\rho}^{k} \partial_{\nu}^{\beta}\left(\partial \tilde{f}^{+}(t)-\mathrm{Id}\right)\right| \leq C \varepsilon_{0}<1 / 2
$$

uniformly with respect to $(r, \theta, \rho, \nu) \in F \Gamma_{s}^{+}\left(\lambda_{0}\right)$, where $\partial \tilde{f}^{+}(t)$ is the differential at $(r, \theta, \rho, \nu)$. Choose $\chi^{+} \in C^{\infty}\left(\mathbb{R}^{2 d}\right)$ so that $0 \leq \chi^{+} \leq 1, \chi^{+} \equiv 1$ on $F \Gamma_{s}^{+}\left(\lambda_{0}\right)$, supp $\chi^{+} \subset F \Gamma_{s}^{+}\left(2 \lambda_{0}\right)$, and that

$$
\left|\partial_{r}^{j} \partial_{\theta}^{\alpha} \partial_{\rho}^{k} \partial_{\nu}^{\beta} \chi^{+}(r, \theta, \rho, \nu)\right| \leq C_{1}\langle r\rangle^{-j} \quad \text { on } \quad \mathbb{R}^{2 d},
$$

and define $\tilde{f}_{\chi}^{+}(t)(r, \theta, \rho, \nu)=\left(r, \theta, \tilde{\rho}_{\chi}(t), \tilde{\omega}_{\chi}(t)\right)$ by

$$
\tilde{f}_{\chi}^{+}(t)(r, \theta, \rho, \nu):=\left(r, \theta,\left(1-\chi^{+}\right) \rho+\chi^{+} \tilde{\rho}(t),\left(1-\chi^{+}\right) \nu+\chi^{+} \tilde{\omega}(t)\right) .
$$

Since $\tilde{f}_{\chi}^{+}(t)=\tilde{f}^{+}(t)$ on $F \Gamma_{s}^{+}\left(\lambda_{0}\right), \tilde{f}_{\chi}^{+}(t)=$ Id outside $F \Gamma_{s}^{+}\left(2 \lambda_{0}\right)$, we have

$$
\left|\partial_{r}^{j} \partial_{\theta}^{\alpha} \partial_{\rho}^{k} \partial_{\nu}^{\beta} \tilde{f}_{\chi}^{+}(t)\right| \leq C, \quad(r, \theta, \rho, \omega) \in \mathbb{R}^{2 d}, j+|\alpha|+k+|\beta| \geq 1 .
$$

Moreover, (5.6) and (5.8) imply

$$
\begin{aligned}
& \left|\partial_{r}^{j} \partial_{\theta}^{\alpha} \partial_{\rho}^{k} \partial_{\nu}^{\beta}\left(\tilde{\rho}_{\chi}(t)-\rho\right)\right| \leq C_{0} C_{1}\langle r\rangle^{-j}|\nu|^{(2-|\beta|)_{+}} \leq C_{2} R_{0}^{-j} \varepsilon_{0}^{(2-|\beta|)_{+}}, \\
& \left|\partial_{r}^{j} \partial_{\theta}^{\alpha} \partial_{\rho}^{k} \partial_{\nu}^{\beta}\left(\tilde{\omega}_{\chi}(t)-\nu\right)\right| \leq C_{0} C_{1}\langle r\rangle^{-j}|\nu|^{(2-|\beta|)_{+}} \leq C_{2} R_{0}^{-j} \varepsilon_{0}^{(2-|\beta|)_{+}}
\end{aligned}
$$

on $\mathbb{R}^{2 d}$ with some $C_{2}>4^{j+2} C_{0} C_{1}$. $\tilde{f}_{\chi}^{+}(t)$ hence satisfies the same estimate as (5.7) on $\mathbb{R}^{2 d}$ provided $R_{0}>0$ large enough and $\varepsilon_{0}>0$ small enough. By the Hadamard global inverse mapping theorem, we see that $\tilde{f}_{\chi}^{+}(t)$ is a diffeomorphism from $\mathbb{R}^{2 d}$ onto its range. Since $f^{+}(t)=$ $F^{-1} \circ \tilde{f}^{+}(t) \circ F, f^{+}(t)$ is a diffeomorphism from $\Gamma_{s}^{+}\left(\lambda_{0}\right)$ onto its range. 
Next, we define $f_{\chi}^{+}(t):=F^{-1} \circ \tilde{f}_{\chi}^{+} \circ F$, and shall prove

$$
\Gamma_{s}^{+}(R, \varepsilon) \subset f_{\chi}^{+}(t)\left(\Gamma_{s}^{+}\left(\lambda_{0}\right)\right), \quad t \geq 0,
$$

for sufficiently large $\lambda_{0}>0$. Since $f_{\chi}^{+}(t)$ is bijective, it suffices to show that

$$
\mathbb{R}^{2 d} \backslash \Gamma_{s}^{+}(R, \varepsilon) \supset f_{\chi}^{+}(t)\left(\mathbb{R}^{2 d} \backslash \Gamma_{s}^{+}\left(\lambda_{0}\right)\right), \quad t \geq 0 .
$$

Suppose that $Z:=(r, \theta, \rho, \omega) \in \mathbb{R}^{2 d} \backslash \Gamma_{s}^{+}\left(\lambda_{0}\right)$. If $Z \in \mathbb{R}^{2 d} \backslash \Gamma_{s}^{+}\left(2 \lambda_{0}\right)$, then

$$
f_{\chi}^{+}(t)(Z)=Z \in \mathbb{R}^{2 d} \backslash \Gamma_{s}^{+}\left(2 \lambda_{0}\right) \subset \mathbb{R}^{2 d} \backslash \Gamma_{s}^{+}(R, \varepsilon) .
$$

If $Z \in \Gamma_{s}^{+}\left(2 \lambda_{0}\right) \backslash \Gamma_{s}^{+}\left(\lambda_{0}\right)$, then we have

$$
p(Z) \in J_{4 \lambda_{0}^{2} \varepsilon^{2}} \backslash J_{\lambda_{0}^{2} \varepsilon^{2}}, \quad \sqrt{1-4 \lambda_{1}^{2} \varepsilon^{2}} \leq \frac{\rho}{\sqrt{2 p(Z)}} \leq \sqrt{1-\lambda_{0}^{2} \varepsilon^{2}} .
$$

Since $\left|p\left(f_{\chi}^{+}(t)(Z)\right)-p(Z)\right| \leq C|\omega / r|^{2} \leq C \varepsilon_{0}^{2}$, Proposition 4.2 and the above argument imply that if $\varepsilon_{0}$ is small enough and $\lambda_{0}$ is large enough, then we obtain

$$
p\left(f_{\chi}^{+}(t)(Z)\right) \notin J_{\varepsilon^{2}}, \quad \frac{\rho_{\chi}(t, Z)}{\sqrt{2 p\left(f_{\chi}^{+}(t)(Z)\right)}}<\sqrt{1-\varepsilon^{2}} \quad \text { for } \quad t \geq 0,
$$

which implies (5.10). Here $\rho_{\chi}(t, Z)=\left(1-\chi^{+}\right) \rho+\chi^{+} \rho(t, Z)$. Since $f_{\chi}^{+}(t)=f^{+}(t)$ on $\Gamma_{s}^{+}\left(\lambda_{0}\right)$, (5.5) follows from (5.10).

Let $\pm t \geq 0$ and $\Gamma_{s}^{ \pm}(R, \varepsilon) \ni(r, \theta, \rho, \omega) \mapsto\left(r, \theta, \hat{\rho}^{ \pm}(t), \hat{\omega}^{ \pm}(t)\right) \in \Gamma_{s}^{ \pm}\left(\lambda_{0}\right)$ the inverse mappings of $f^{ \pm}(t)$, respectively.

Lemma 5.4. For $0 \leq \pm s \leq \pm t$ and $(r, \theta, \rho, \omega) \in \Gamma_{s}^{ \pm}(R, \varepsilon)$, we define

$$
\left(r_{t}^{ \pm}(s), \theta_{t}^{ \pm}(s), \rho_{t}^{ \pm}(s), \omega_{t}^{ \pm}(s)\right):=(r, \theta, \rho, \omega)\left(s, r, \theta,\left(\hat{\rho}^{ \pm}, \hat{\omega}^{ \pm}\right)(t, r, \theta, \rho, \omega)\right) .
$$

We then have, for all $(j, \alpha, k, \beta) \in \mathbb{Z}_{+}^{2 d}$,

$$
\begin{gathered}
\left|\partial_{r}^{j} \partial_{\theta}^{\alpha} \partial_{\rho}^{k} \partial_{\omega}^{\beta}\left(\hat{\rho}^{ \pm}(t)-\rho\right)\right| \leq C r^{-j-|\beta|}|\omega / r|^{(2-|\beta|)_{+}}, \\
\left|\partial_{r}^{j} \partial_{\theta}^{\alpha} \partial_{\rho}^{k} \partial_{\omega}^{\beta}\left(\hat{\omega}^{ \pm}(t)-\omega\right)\right| \leq C r^{1-j-|\beta|}|\omega / r|^{(2-|\beta|)_{+}},
\end{gathered}
$$

uniformly with respect to $(r, \theta, \rho, \omega) \in \Gamma_{s}^{ \pm}(R, \varepsilon)$ and $\pm t \geq 0$.

Proof. Since $\left(r, \theta, \hat{\rho}^{ \pm}(t), \hat{\omega}^{ \pm}(t)\right) \in \Gamma_{s}^{ \pm}\left(\lambda_{0}\right)$, we have

$$
\begin{aligned}
\left|\hat{\rho}^{ \pm}(t)-\rho\right|+r^{-1}\left|\hat{\omega}^{ \pm}(t)-\omega\right| & =\left|\rho_{t}(0)-\rho_{t}^{ \pm}(t)\right|+r^{-1}\left|\omega_{t}(0)-\omega_{t}^{ \pm}(t)\right| \\
& \leq C \sup _{\Gamma_{s}^{ \pm}\left(\lambda_{0}\right)}\left(|\rho(t)-\rho|+r^{-1}|\omega(t)-\omega|\right) \\
& \leq C|\omega / r|^{2}
\end{aligned}
$$

where $C$ is independent of $R_{0}, \varepsilon_{0}$ and $\lambda_{0}$. We next consider the derivatives. Let $\gamma=(j, \alpha, k, \beta)$, $|\gamma|=1$. Applying $\partial^{\gamma}=\partial_{r}^{j} \partial_{\theta}^{\alpha} \partial_{\rho}^{k} \partial_{\omega}^{\beta}$ to the equality

$$
(\rho, \omega)=(\rho, \omega)\left(t, r, \theta, \hat{\rho}^{ \pm}(t), \hat{\omega}^{ \pm}(t)\right),
$$

we have

$$
A\left(Z^{ \pm}(t)\right)\left(\begin{array}{c}
\partial^{\gamma}\left(\hat{\rho}^{ \pm}(t)-\rho\right) \\
r^{-1} \partial^{\gamma}\left(\hat{\omega}^{ \pm}(t)-\omega\right)
\end{array}\right)=\left.\left(\begin{array}{c}
\partial^{\gamma}(\rho-\rho(t)) \\
r^{-1} \partial^{\gamma}(\omega-\omega(t))
\end{array}\right)\right|_{Z^{ \pm}(t)}
$$

where $Z^{ \pm}(t)=\left(r, \theta, \hat{\rho}^{ \pm}(t), \hat{\omega}^{ \pm}(t)\right)$ and

$$
A\left(Z^{ \pm}(t)\right)=\left(\begin{array}{cc}
\left(\partial_{\rho} \rho\right)\left(t, Z^{ \pm}(t)\right) & r\left(\partial_{\omega} \rho\right)\left(t, Z^{ \pm}(t)\right) \\
r^{-1}\left(\partial_{\rho} \omega\right)\left(t, Z^{ \pm}(t)\right) & \left(\partial_{\omega} \omega\right)\left(t, Z^{ \pm}(t)\right)
\end{array}\right) .
$$

(4.3) and (5.11) show that $A\left(Z^{ \pm}(t)\right)$ are invertible, $A\left(Z^{ \pm}(t)\right)$ and $A\left(Z^{ \pm}(t)\right)^{-1}$ are bounded on $\Gamma_{s}^{ \pm}(R, \varepsilon)$ and the right hand side of $(5.12)$ is bounded by

$$
r^{-j-|\beta|}|\omega / r|^{(2-|\beta|)+} .
$$


The proof for higher derivatives is obtained by a simple induction with respect to $|\gamma|$, and we omit the details.

The following easily follows from Lemma 4.1, Proposition 4.2 and Lemma 5.4.

Corollary 5.5. For all $\pm t \geq 0,(r, \theta, \rho, \omega) \in \Gamma_{s}^{ \pm}(R, \varepsilon)$, we have

$$
r_{t}^{ \pm}(t) \geq C^{-1}(r+|t|) .
$$

Moreover, for all $(j, \alpha, k, \beta) \in \mathbb{Z}_{+}^{2 d}$,

$$
\begin{aligned}
\left|\partial_{r}^{j} \partial_{\theta}^{\alpha} \partial_{\rho}^{k} \partial_{\omega}^{\beta}\left(r_{t}^{ \pm}(t)-r \mp t \rho\right)\right| & \leq C r^{1-j-|\beta|}|\omega / r|^{(2-|\beta|)_{+}}, \\
\left|\partial_{r}^{j} \partial_{\theta}^{\alpha} \partial_{\rho}^{k} \partial_{\omega}^{\beta}\left(\theta_{t}^{ \pm}(t)-\theta\right)\right| & \leq C r^{-j-|\beta|}|\omega / r|^{(1-|\beta|)_{+}} .
\end{aligned}
$$

Proof of Theorem 5.1. We give the proof for the case $t \geq 0$, and the proof for the case $t \leq 0$ is analogous. Define $\Lambda^{+}(t) \in C^{\infty}\left(\Gamma_{s}^{+}(R, \varepsilon)\right)$ for $t \geq 0$ by

$$
\Lambda^{+}(t, r, \theta, \rho, \omega)=r_{t}^{+}(t) \rho+\theta_{t}^{+}(t) \cdot \omega+\int_{0}^{t} L\left(r_{t}^{+}(s), \theta_{t}^{+}(s), \rho_{t}^{+}(s), \omega_{t}^{+}(s)\right) d s,
$$

where $L(r, \theta, \rho, \omega)=\partial_{\rho} p(r, \theta, \rho, \omega) \rho+\partial_{\omega} p(r, \theta, \rho, \omega) \cdot \omega-p(r, \theta, \rho, \omega)$ is the Lagrangian associated with $p$. Note that the smoothness of $\Lambda^{+}(t)$ follows from the smoothness of $\left(r_{t}^{+}, \theta_{t}^{+}, \rho_{t}^{+}, \omega_{t}^{+}\right)$. By the standard Hamilton-Jacobi theory, $\Lambda^{+}(t)$ solves

$$
\left\{\begin{aligned}
\partial_{t} \Lambda^{+}(t) & =p\left(r, \theta, \partial_{r} \Lambda^{+}(t), \partial_{\theta} \Lambda^{+}(t)\right), \\
\Lambda^{+}(0) & =r \rho+\theta \cdot \omega, \\
\partial_{r, \theta, \rho, \omega} \Lambda^{+}(t) & =\left(\hat{\rho}^{+}(t), \hat{\omega}^{+}(t), r_{t}^{+}(t), \theta_{t}^{+}(t)\right) .
\end{aligned}\right.
$$

Put $F^{+}(t)=\Lambda^{+}(t)-\frac{1}{2} t \rho^{2}$. The energy conservation, namely

$$
p\left(r, \theta, \hat{\rho}^{+}(t), \hat{\omega}^{+}(t)\right)=p\left(r_{t}^{+}(t), \theta_{t}^{+}(t), \rho, \omega\right),
$$

implies

$$
\partial_{t} F^{+}(t)=\frac{1}{2 r_{t}^{+}(t)^{2}}\left(h^{j k}\left(\theta_{t}^{+}(t)\right)+a^{j k}\left(r_{t}^{+}(t), \theta_{t}^{+}(t)\right) \omega_{j} \omega_{k}^{0} .\right.
$$

By Lemma 5.4 and Corollary 5.5, we have

$$
\left|\partial_{r}^{j} \partial_{\theta}^{\alpha} \partial_{\rho}^{k} \partial_{\omega}^{\beta} \partial_{t} F^{+}(t)\right| \leq C(r+|t|)^{-2-j} r^{2-|\beta|}|\omega / r|^{(2-|\beta|)_{+}},
$$

and hence

$$
\left|\int_{0}^{\infty} \partial_{r}^{j} \partial_{\theta}^{\alpha} \partial_{\rho}^{k} \partial_{\omega}^{\beta} \partial_{t} F^{+}(t) d t\right| \leq C r^{1-j-|\beta|}|\omega / r|^{(2-|\beta|)+}
$$

for all $t \geq 0,(r, \theta, \rho, \omega) \in \Gamma_{s}^{+}(R, \varepsilon)$ and $(j, \alpha, k, \beta) \in \mathbb{Z}_{+}^{2 d}$. If we put $q_{0}(\theta, \omega)=h^{j k}(\theta) \omega_{j} \omega_{k}$, then the mean value theorem and Corollary 5.5 imply

$$
\begin{aligned}
& \left|\partial_{r}^{j} \partial_{\theta}^{\alpha} \partial_{\rho}^{k} \partial_{\omega}^{\beta}\left(\frac{1}{2 r_{t}^{+}(t)^{2}} q_{0}\left(\theta_{t}^{+}(t), \omega\right)-\frac{1}{2(r+t \rho)^{2}} q_{0}(\theta, \omega)\right)\right| \\
& \leq C(r+|t|)^{-2} r^{2-j-|\beta|}|\omega / r|^{(3-|\beta|)_{+}} .
\end{aligned}
$$

Therefore, $\partial_{t} F^{+}(t)$ can be written in the form

$$
\frac{1}{2(r+t \rho)^{2}} q_{0}(\theta, \omega)+\tilde{R}^{+}(t)
$$

with $\tilde{R}^{+}(t) \in C^{\infty}\left(\Gamma^{+}(R, \varepsilon)\right)$ satisfying

$$
\begin{aligned}
& \left|\partial_{r}^{j} \partial_{\theta}^{\alpha} \partial_{\rho}^{k} \partial_{\omega}^{\beta} \tilde{R}^{+}(t, r, \theta, \rho, \omega)\right| \\
& \leq C(r+|t|)^{-2} r^{2-j-|\beta|}\left(|\omega / r|^{(3-|\beta|)_{+}}+r^{-\mu}|\omega / r|^{(2-|\beta|)_{+}}\right) .
\end{aligned}
$$


Define $\tilde{\varphi}^{+}, \tilde{S}^{+}$on $\Gamma_{s}^{+}(R, \varepsilon)$ by

$$
\begin{aligned}
& \tilde{\varphi}^{+}(r, \theta, \rho, \omega):=\int_{0}^{\infty} \partial_{t} F^{+}(t, r, \theta, \rho, \omega) d t, \\
& \tilde{S}^{+}(r, \theta, \rho, \omega):=r \rho+\theta \cdot \omega+\tilde{\varphi}^{+}(r, \theta, \rho, \omega) .
\end{aligned}
$$

The above argument shows that $\tilde{\varphi}^{+}, \tilde{S}^{+}$are smooth and $\tilde{\varphi}^{+}$satisfies (5.1) and (5.2) on $\Gamma_{s}^{+}(R, \varepsilon)$. Moreover, we have

$$
\partial_{r, \theta} \tilde{S}^{+}=\lim _{t \rightarrow+\infty} \partial_{r, \theta} \Lambda^{+}(t) \quad \text { on } \quad \Gamma_{s}^{+}(R, \varepsilon) .
$$

By using the energy conservation and Corollary 5.5, we see that $\tilde{S}^{+}$satisfies the Eikonal equation on $\Gamma_{s}^{+}(R, \varepsilon)$ :

$$
\begin{aligned}
p\left(r, \theta, \partial_{r} \tilde{S}^{+}, \partial_{\theta} \tilde{S}^{+}\right) & =\lim _{t \rightarrow+\infty} p\left(r, \theta, \partial_{r} \Lambda^{+}(t), \partial_{\theta} \Lambda^{+}(t)\right) \\
& =\lim _{t \rightarrow+\infty} p\left(r_{t}^{+}(t), \theta_{t}^{+}(t), \rho, \omega\right) \\
& =\rho^{2} / 2 .
\end{aligned}
$$

Choosing a smooth cut-off function $\chi^{+}$on $\mathbb{R}^{2 d}$ so that $0 \leq \chi^{+} \leq 1, \chi^{+} \equiv 1$ on $\Gamma_{s}^{+}(R, \varepsilon)$, $\operatorname{supp} \chi^{+} \subset \Gamma_{s}^{+}(\lambda), \lambda \geq \lambda_{0}$ and that

$$
\left|\partial_{r}^{j} \partial_{\theta}^{\alpha} \partial_{\rho}^{k} \partial_{\omega}^{\beta} \chi(r, \theta, \rho, \omega)\right| \leq C\langle r\rangle^{-j-|\beta|} \quad \text { on } \quad \mathbb{R}^{2 d},
$$

we define $\varphi^{+}, S^{+} \in C^{\infty}\left(\mathbb{R}^{2 d}\right)$ by $\varphi^{+}=\chi^{+} \tilde{\varphi}^{+}, S^{+}:=r \rho+\theta \cdot \omega+\varphi^{+}$. Clearly, $\varphi^{+}$and $S^{+}$satisfy the statements of Theorem 5.1.

Definition 5.6. For $a^{ \pm} \in S_{\mathrm{sc}}\left(\Gamma^{ \pm}(R, \varepsilon)\right)$ and $h \in(0,1]$, we define the FIO's for the IsozakiKitada parametrix by

$$
I_{\mathrm{IK}}^{ \pm}\left(a^{ \pm}\right) u(r, \theta)=(2 \pi h)^{-d} \int e^{\frac{i}{h}\left(S^{ \pm}(r, \theta, \rho, \omega)-r^{\prime} \rho-\theta^{\prime} \cdot \omega\right)} a^{ \pm}(r, \theta, \rho, \omega) u\left(r^{\prime}, \theta^{\prime}\right) d r^{\prime} d \theta^{\prime} d \rho d \omega .
$$

The following theorem shows that $I_{\mathrm{IK}}^{ \pm}\left(a^{ \pm}\right)$are bounded on $L^{2}\left(\mathbb{R}^{d}\right)$ uniformly with respect to $R>0$ and $h \in(0,1]$.

Proposition 5.7. Let $R_{0}, \varepsilon_{0}, \lambda_{0}>0$ be as in Theorem 5.1, $\lambda \geq \lambda_{0}, R \geq \lambda^{4} R_{0}$ and $0<\varepsilon \leq$ $\lambda^{-4} \varepsilon_{0}$. Then, for all $N \geq 0$ and $a^{ \pm}, b^{ \pm} \in S_{\mathrm{sc}}\left(\Gamma^{ \pm}\left(\lambda^{3}\right)\right)$, there exist symbols $c_{j}^{ \pm} \in S_{\mathrm{sc}}\left(\Gamma^{ \pm}\left(\lambda^{4}\right)\right), j=$ $0,1, \ldots, N$, such that if we put $c_{h}^{ \pm}=\sum_{j=0}^{N} h^{j} c_{j}^{ \pm}$, then

$$
\left\|I_{\mathrm{IK}}^{ \pm}\left(a^{ \pm}\right) I_{\mathrm{IK}}^{ \pm}\left(b^{ \pm}\right)^{*}-c_{h}^{ \pm}\left(r, \theta, h D_{r}, h D_{\theta}\right)\right\|_{\mathcal{L}\left(L^{2}\left(\mathbb{R}^{d}\right)\right)} \leq C_{N} h^{N+1}, \quad h \in(0,1] .
$$

In particular, we have

$$
\left\|I_{\mathrm{IK}}^{ \pm}\left(a^{ \pm}\right)\right\|_{\mathcal{L}\left(L^{2}\left(\mathbb{R}^{d}\right)\right)} \leq C, \quad h \in(0,1] .
$$

Here $C_{N}, C>0$ may be taken uniformly with respect to $R$ and $h$.

The following shows that any elliptic FIO (in the semiclassical sense) has a microlocal approximate inverse.

Proposition 5.8. Let $R_{0}, \varepsilon_{0}, \lambda_{0}>0$ be as in Theorem 5.1, $\lambda \geq \lambda_{0}, R \geq \lambda^{4} R_{0}, 0<\varepsilon \leq$ $\lambda^{-4} \varepsilon_{0}$ and $N \geq 0$ a non-negative integer. Choose arbitrarily sequences of symbols $\left(a_{j}^{ \pm}\right)_{0 \leq j \leq N} \subset$ $S_{\mathrm{sc}}\left(\Gamma^{ \pm}\left(\lambda^{3}\right)\right)$ satisfying

$$
a_{0}^{ \pm}>C_{0}^{-1} \quad \text { on } \quad \Omega_{s}^{ \pm}(\lambda)
$$

with some fixed $C_{0}>0$, respectively. Then for all $c^{ \pm} \in S_{\mathrm{sc}}\left(\Gamma^{ \pm}(R, \varepsilon)\right)$, there exist sequences of symbols $\left(b_{j}^{ \pm}\right)_{0 \leq j \leq N} \subset S_{\mathrm{sc}}\left(\Gamma^{ \pm}(\lambda)\right)$ such that

$$
\left\|I_{\mathrm{IK}}^{ \pm}\left(a^{ \pm}\right) I_{\mathrm{IK}}^{ \pm}\left(b^{ \pm}\right)^{*}-c^{ \pm}\left(r, \theta, h D_{r}, h D_{\theta}\right)\right\|_{\mathcal{L}\left(L^{2}\left(\mathbb{R}^{d}\right)\right)} \leq C_{N} h^{N+1}, h \in(0,1],
$$

where $a_{h}^{ \pm}=\sum_{j=0}^{N} h^{j} a_{j}^{ \pm}$and $b_{h}^{ \pm}=\sum_{j=0}^{N} h^{j} b_{j}^{ \pm}$. Moreover $C_{N}>0$ can be taken uniformly with respect to $R$ and $h$. 
To prove the above two propositions, we need the following lemma.

Lemma 5.9. Define $\left(\rho_{1}^{ \pm}, \omega_{1}^{ \pm}\right)\left(r, \theta, \rho, \omega, r^{\prime}, \theta^{\prime}\right): \mathbb{R}^{3 d} \rightarrow \mathbb{R}^{d}$ by

$$
\left(\rho_{1}^{ \pm}, \omega_{1}^{ \pm}\right)=\int_{0}^{1}\left(\partial_{r, \theta} S^{ \pm}\right)\left(r^{\prime}+\sigma\left(r-r^{\prime}\right), \theta^{\prime}+\sigma\left(\theta-\theta^{\prime}\right), \rho, \omega\right) d \sigma .
$$

Then the followings hold for all $\lambda \geq \lambda_{0}, R \geq \lambda R_{0}$ and $0<\varepsilon \leq \varepsilon_{0} / \lambda$.

(i) If $(r, \theta, \rho, \omega) \in \Gamma_{s}^{ \pm}(R, \varepsilon)$, then

$$
\left.\left(r, \theta, \rho_{1}^{ \pm}, \omega_{1}^{ \pm}\right)\right|_{r^{\prime}=r, \theta^{\prime}=\theta} \in \Gamma_{s}^{ \pm}(\lambda) .
$$

Conversely, if $\left.\left(r, \theta, \rho_{1}^{ \pm}, \omega_{1}^{ \pm}\right)\right|_{r^{\prime}=r, \theta^{\prime}=\theta} \in \Gamma_{s}^{ \pm}(R, \varepsilon)$, then

$$
(r, \theta, \rho, \omega) \in \Gamma_{s}^{ \pm}(\lambda) .
$$

(ii) We set $\widetilde{\Gamma}_{s}^{ \pm}(\lambda):=\left\{\left(r, \theta, \rho, \omega, r^{\prime}, \theta^{\prime}\right) \in \mathbb{R}^{3 d} ;(r, \theta, \rho, \omega),\left(r^{\prime}, \theta^{\prime}, \rho, \omega\right) \in \Gamma^{ \pm}(\lambda)\right\}$ and $\widetilde{\Gamma}_{s}^{ \pm}(R, \varepsilon):=$ $\widetilde{\Gamma}_{s}^{ \pm}(1)$. Then, the map $\left(r, \theta, \rho, \omega, r^{\prime}, \theta^{\prime}\right) \mapsto\left(r, \theta, \rho_{1}^{ \pm}, \omega_{1}^{ \pm}, r^{\prime}, \theta^{\prime}\right)$ is a diffeomorphism from $\widetilde{\Gamma}_{s}^{ \pm}(\lambda)$ onto its range, and satisfies

$$
\pi_{\rho, \omega}\left(\widetilde{\Gamma}_{s}^{ \pm}(R, \varepsilon)\right) \subset\left(\rho_{1}^{ \pm}, \omega_{1}^{ \pm}\right)\left(\pi_{\rho, \omega}\left(\widetilde{\Gamma}_{s}^{ \pm}(\lambda)\right)\right),
$$

respectively. Denoting by $\left(\rho_{2}^{ \pm}, \omega_{2}^{ \pm}\right)$the corresponding inverse, the same properties as in (i) hold with $\left(\rho_{1}^{ \pm}, \omega_{1}^{ \pm}\right)$replaced by $\left(\rho_{2}^{ \pm}, \omega_{2}^{ \pm}\right)$, respectively.

(iii) If $\lambda, \lambda^{\prime} \geq \lambda_{0}, R \geq \max \left(\lambda, \lambda^{\prime}\right) R_{0}$ and $0<\varepsilon \leq \min \left(\lambda^{-1}, \lambda^{\prime-1}\right) \varepsilon_{0}$, then we have

$$
\begin{aligned}
& \left|\partial^{\gamma}\left(\rho_{2}^{ \pm}-\rho\right)\right|+\min \left(r, r^{\prime}\right)^{-1}\left|\partial^{\gamma}\left(\omega_{2}^{ \pm}-\omega\right)\right| \\
& \leq C \min \left(r, r^{\prime}\right)^{-j-j^{\prime}-|\beta|}\left(\max \left(\lambda, \lambda^{\prime}\right) \varepsilon\right)^{(2-|\beta|)_{+},}
\end{aligned}
$$

for all $\left(r, \theta, \rho_{2}^{ \pm}, \omega_{2}^{ \pm}\right) \in \Gamma_{s}^{ \pm}(\lambda)$ and $\left(r^{\prime}, \theta^{\prime}, \rho_{2}^{ \pm}, \omega_{2}^{ \pm}\right) \in \Gamma_{s}^{ \pm}\left(\lambda^{\prime}\right)$, where we use the notation $\partial^{\gamma}:=$ $\partial_{r}^{j} \partial_{\theta}^{\alpha} \partial_{\rho}^{k} \partial_{\omega}^{\beta} \partial_{r^{\prime}}^{j^{\prime}} \partial_{\theta^{\prime}}^{\alpha^{\prime}}$ for $\gamma=\left(j, \alpha, k, \beta, j^{\prime}, \alpha^{\prime}\right) \in \mathbb{Z}_{+}^{3 d}$.

Proof. We only consider the outgoing case. Remark that (5.14) is equivalent to

$$
\left(\rho_{1}^{+}, \omega_{1}^{+}\right)=(\rho, \omega)+\int_{0}^{1}\left(\partial_{r, \theta} \varphi^{+}\right)\left(r^{\prime}+\sigma\left(r-r^{\prime}\right), \theta^{\prime}+\sigma\left(\theta-\theta^{\prime}\right), \rho, \omega\right) d \sigma .
$$

Suppose that $(r, \theta, \rho, \omega) \in \Gamma_{s}^{+}(R, \varepsilon)$. Since

$$
\left.\left(\rho_{1}^{+}-\rho, \omega_{1}^{+}-\omega\right)\right|_{r^{\prime}=r, \theta^{\prime}=\theta}=\left(\partial_{r, \theta} \varphi^{+}\right)(r, \theta, \rho, \omega),
$$

(5.1) implies $\left.\left(\left|\rho_{1}^{+}-\rho\right|+r^{-1}\left|\omega_{1}^{+}-\omega\right|\right)\right|_{r^{\prime}=r, \theta^{\prime}=\theta} \leq C \varepsilon^{2}$, and

$$
\left|p_{1}-p\right| \leq C p \varepsilon^{2},\left.\quad \rho_{1}^{+}\right|_{r^{\prime}=r, \theta^{\prime}=\theta} \geq \sqrt{\left(1-C \varepsilon^{2}\right) 2 p_{1}},
$$

with some $C>0$ which is independent of $R, \varepsilon$ and $\lambda_{0}$, where we denote $p=p(r, \theta, \rho, \omega)$ and $p_{1}=\left.p\left(r, \theta, \rho_{1}^{+}, \omega_{1}^{+}\right)\right|_{r^{\prime}=r, \theta^{\prime}=\theta}$. Choosing $\lambda>0$ large enough such that $\lambda^{2}>C$, we have $\left.\left(r, \theta, \rho_{1}^{+}, \omega_{1}^{+}\right)\right|_{r^{\prime}=r, \theta^{\prime}=\theta} \in \Gamma^{+}(\lambda)$. Next, consider the mapping

$$
f^{+}:\left.(r, \theta, \rho, \omega) \mapsto\left(r, \theta, \rho_{1}^{+}, \omega_{1}^{+}\right)\right|_{r^{\prime}=r, \theta^{\prime}=\theta} .
$$

By (5.1), we have

$$
\begin{aligned}
\left|\partial_{r}^{j} \partial_{\theta}^{\alpha} \partial_{\rho}^{k} \partial_{\omega}^{\beta}\left(\left.\rho_{1}^{+}\right|_{r^{\prime}=r, \theta^{\prime}=\theta}-\rho\right)\right| \leq C_{j \alpha k \beta} r^{-j-|\beta|}|\omega / r|^{(2-|\beta|)_{+}} \\
\left|\partial_{r}^{j} \partial_{\theta}^{\alpha} \partial_{\rho}^{k} \partial_{\omega}^{\beta}\left(\left.\omega_{1}^{+}\right|_{r^{\prime}=r, \theta^{\prime}=\theta}-\omega\right)\right| \leq C_{j \alpha k \beta} r^{1-j-|\beta|}|\omega / r|^{(2-|\beta|)_{+}}
\end{aligned}
$$

By same argument as that in the proof of Lemma 5.3, we obtain that $f^{+}$is injective and $\Gamma_{s}^{+}(R, \varepsilon) \subset f^{+}\left(\Gamma_{s}^{+}(\lambda)\right)$ provided that $\lambda>0$ is large enough. We note that this $\lambda$ can be taken uniformly with respect to $R$ and $\varepsilon$. This fact implies (5.16). 
We next prove (ii). We write $\left(r_{\sigma}, \theta_{\sigma}\right)=\left(r^{\prime}+\sigma\left(r-r^{\prime}\right), \theta^{\prime}+\sigma\left(\theta-\theta^{\prime}\right)\right)$ for short. Since $|\omega| \lesssim \min \left(r, r^{\prime}\right) \lambda \varepsilon,(5.1)$ implies, for $\gamma=\left(j, \alpha, k, \beta, j^{\prime}, \alpha^{\prime}\right) \in \mathbb{Z}_{+}^{3 d}$,

$$
\begin{aligned}
\left|\left(\partial^{\gamma} \varphi^{+}\right)\left(r_{\sigma}, \theta_{\sigma}, \rho, \omega\right)\right| & \leq C_{\gamma} r_{\sigma}^{1-j-|\beta|}\left|\omega / r_{\sigma}\right|^{(2-|\beta|)_{+}} \\
& \leq C_{\gamma} \min \left(r^{1-j-|\beta|},\left(r^{\prime}\right)^{1-j-|\beta|}\right)(\lambda \varepsilon)^{(2-|\beta|)_{+}},
\end{aligned}
$$

and hence

$$
\left|\partial^{\gamma}\left(\rho_{1}^{+}-\rho\right)\right|+\frac{\left|\partial^{\gamma}\left(\omega_{1}^{+}-\omega\right)\right|}{\min \left(r, r^{\prime}\right)} \leq C_{\gamma} R_{0}^{-j-|\beta|}(\lambda \varepsilon)^{(2-|\beta|)_{+}} \leq C_{\gamma} R_{0}^{-j-|\beta|}\left(\lambda \varepsilon \varepsilon_{0}\right)^{(1-|\beta|)_{+}} .
$$

In particular, $\left(r, \theta, \rho_{1}^{ \pm}, \omega_{1}^{ \pm}, r^{\prime}, \theta^{\prime}\right) \in \widetilde{\Gamma}_{s}^{+}\left(\lambda^{2}\right)$ if $\lambda>0$ is large enough. By a same argument as that in the proof of Lemma 5.3, we obtain the first assertion of (ii). Applying (5.16) and (5.15) with $(\rho, \omega)=\left.\left(\rho_{2}^{+}, \omega_{2}^{+}\right)\right|_{r^{\prime}=r, \theta^{\prime}=\theta}$, we obtain

$$
\begin{aligned}
&\left.\left(r, \theta, \rho_{2}^{+}, \omega_{2}^{+}\right)\right|_{r^{\prime}=r, \theta^{\prime}=\theta} \in \Gamma_{s}^{+}(\lambda) \text { if } \quad(r, \theta, \rho, \omega) \in \Gamma_{s}^{+}(R, \varepsilon), \\
&(r, \theta, \rho, \omega) \in \Gamma_{s}^{+}(\lambda) \quad \text { if }\left.\quad\left(r, \theta, \rho_{2}^{+}, \omega_{2}^{+}\right)\right|_{r^{\prime}=r, \theta^{\prime}=\theta} \in \Gamma_{s}^{+}(R, \varepsilon) .
\end{aligned}
$$

Finally we shall prove (iii). Since $\left(r, \theta, \rho_{2}^{+}, \omega_{2}^{+}\right)$satisfy

$$
\left(\rho_{2}^{+}, \omega_{2}^{+}\right)=(\rho, \omega)-\int_{0}^{1}\left(\partial_{r, \theta} \varphi^{+}\right)\left(r^{\prime}+\sigma\left(r-r^{\prime}\right), \theta^{\prime}+\sigma\left(\theta-\theta^{\prime}\right), \rho_{2}^{+}, \omega_{2}^{+}\right) d \sigma,
$$

(5.1) implies

$$
\left(\rho_{2}^{+}-\rho\right)\left|+\min \left(r, r^{\prime}\right)^{-1}\right| \omega_{2}^{+}-\omega \mid \leq C \max \left(\lambda, \lambda^{\prime}\right)^{2} \varepsilon^{2}
$$

for $\left(r, \theta, \rho_{2}^{+}, \omega_{2}^{+}\right) \in \Gamma_{s}^{+}(\lambda)$ and $\left(r^{\prime}, \theta^{\prime}, \rho_{2}^{+}, \omega_{2}^{+}\right) \in \Gamma_{s}^{+}\left(\lambda^{\prime}\right)$. For the derivatives, differentiating (5.20) with respect to $\partial_{r}^{j} \partial_{\theta}^{\alpha} \partial_{r^{\prime}}^{j^{\prime}} \partial_{\theta^{\prime}}^{\alpha^{\prime}} \partial_{\rho}^{k} \partial_{\omega}^{\beta}$ and using (5.1), we obtain (5.17) by an induction with respect to $j+|\alpha|+k+|\beta|+j^{\prime}+\left|\alpha^{\prime}\right|$.

Proof of Proposition 5.7. We only prove the outgoing case. Note that since $R \geq \lambda^{4} R_{0}$ and $\varepsilon \leq \lambda^{-4} \varepsilon_{0},\left(\rho_{2}^{+}, \omega_{2}^{+}\right)$is well-defined for $r, r^{\prime}>\lambda^{-3} R$. The Schwartz kernel of $I_{\mathrm{IK}}^{+}\left(a^{+}\right) I_{\mathrm{IK}}^{+}\left(b^{+}\right)^{*}$ can be written in the form

$$
(2 \pi h)^{-d} \int e^{\frac{i}{h}\left(S^{+}(r, \theta, \rho, \omega)-S^{+}\left(r^{\prime}, \theta^{\prime}, \rho, \omega\right)\right)} a^{+}(r, \theta, \rho, \omega) \overline{b^{+}\left(r^{\prime}, \theta^{\prime}, \rho, \omega\right)} d \rho d \omega .
$$

Since $\left(r, \theta, \rho, \omega, r^{\prime}, \theta^{\prime}\right) \in \widetilde{\Gamma}_{s}^{+}\left(\lambda^{4}\right)$, Lemma 5.9 implies that $\left(\rho_{2}^{+}, \omega_{2}^{+}\right)$is well-defined and

$$
1 / 2<\left|\operatorname{det} \partial_{\rho, \omega}\left(\rho_{2}^{+}, \omega_{2}^{+}\right)\right|<3 / 2 .
$$

for all $\left(r, \theta, \rho, \omega, r^{\prime}, \theta^{\prime}\right)$ with $\left(r, \theta, \rho_{2}^{+}, \omega_{2}^{+}, r^{\prime}, \theta^{\prime}\right) \in \widetilde{\Gamma}_{s}^{+}\left(\lambda^{4}\right)$. We thus can make the change of variables $(\rho, \omega) \mapsto\left(\rho_{2}^{+}, \omega_{2}^{+}\right)\left(r, \theta, \rho, \omega, r^{\prime}, \theta^{\prime}\right)$, and the above integral can be brought to the form

$$
(2 \pi h)^{-d} \int e^{\frac{i}{h}\left[\left(r-r^{\prime}\right) \rho-\left(\theta-\theta^{\prime}\right) \cdot \omega\right]} A^{+}\left(r, \theta, \rho, \omega, r^{\prime}, \theta^{\prime}\right) d \rho d \omega,
$$

where $A^{+}=a^{+}\left(r, \theta, \rho_{2}^{+}, \omega_{2}^{+}\right) \overline{b^{+}\left(r^{\prime}, \theta^{\prime}, \rho_{2}^{+}, \omega_{2}^{+}\right)}\left|\operatorname{det} \partial_{\rho, \omega}\left(\rho_{2}^{+}, \omega_{2}^{+}\right)\right|$. By using (5.17), (5.21) and the support properties of $a^{+}$and $b^{+}, \partial_{r}^{j} \partial_{\theta}^{\alpha} \partial_{\rho}^{k} \partial_{\omega}^{\beta} \partial_{r^{\prime}}^{j^{\prime}} \partial_{\theta^{\prime}}^{\alpha^{\prime}} A^{+}$are uniformly bounded on $\mathbb{R}^{3 d}$ for all $\left(j, \alpha, k, \beta, j^{\prime}, \alpha^{\prime}\right) \in \mathbb{Z}_{+}^{3 d}$, and the Calderón-Vaillancourt theorem implies

$$
\left\|I_{\mathrm{IK}}^{+}\left(a^{+}\right) I_{\mathrm{IK}}^{+}\left(b^{+}\right)^{*}\right\|_{\mathcal{L}\left(L^{2}\left(\mathbb{R}^{d}\right)\right)} \leq C_{d} \sum_{|\gamma| \leq M_{d}}\left\|\partial^{\gamma} A^{+}\right\|_{L^{\infty}\left(\mathbb{R}^{3 d}\right)} \leq C
$$

uniformly with respect to $R>0$ and $h \in(0,1]$, with some $C_{d}, M_{d}>0$ depending only on d. In particular $I_{\mathrm{IK}}^{+}\left(a^{+}\right)_{\mathcal{L}\left(L^{2}\left(\mathbb{R}^{d}\right)\right)}$ is bounded on $L^{2}\left(\mathbb{R}^{d}\right)$ uniformly with respect to $R>0$ and $h \in(0,1]$. Furthermore, by the standard symbolic calculus (e.g., see the textbook [15]), the simplified symbol of the above operator has the following asymptotic expansion

$$
\sum_{l+|\alpha|=j} \frac{h^{j}}{l ! \alpha !}\left(\partial_{\rho}^{l} \partial_{\omega}^{\alpha} D_{r^{\prime}}^{l} D_{\theta^{\prime}}^{\alpha} A^{+}\right)(r, \theta, \rho, \omega, r, \theta) .
$$


By Lemma 5.9 (ii) and (iii), we see that $\left(\partial_{\rho}^{l} \partial_{\omega}^{\alpha} D_{r^{\prime}}^{l} D_{\theta^{\prime}}^{\alpha} A^{+}\right)(r, \theta, \rho, \omega, r, \theta)$ is supported in $\Gamma_{s}^{+}\left(\lambda^{4}\right)$ and belongs to $S_{\mathrm{sc}}\left(\Gamma^{+}\left(\lambda^{4}\right)\right)$.

Proof of Proposition 5.8. Let $c^{+} \in S_{\mathrm{sc}}\left(\Gamma^{+}(R, \varepsilon)\right)$. By Proposition 5.7, it suffices to show that there exist $b_{j}^{+} \in S_{\mathrm{sc}}\left(\Gamma^{+}(\lambda)\right), j=0,1, \ldots, N$, such that $c_{0}^{+}=c^{+}$and $c_{j}^{+} \equiv 0$ for $j=1,2, \ldots, N$. We set $J_{k}=\left|\operatorname{det} \partial_{\rho, \omega}\left(\rho_{k}^{+}, \omega_{k}^{+}\right)\right| . b_{j}^{+}$can be defined inductively as follows. We first note that, by the construction, $b_{h}^{+}$should satisfy

$$
\begin{aligned}
& c^{+}(r, \theta, \rho, \omega) \\
& =\left.\sum_{j=1}^{N} \sum_{l+|\alpha|=j} \frac{h^{j}}{l ! \alpha !}\left(\partial_{\rho}^{l} \partial_{\omega}^{\alpha} D_{r^{\prime}}^{l} D_{\theta^{\prime}}^{\alpha} a_{h}^{+}\left(r, \theta, \rho_{2}^{+}, \omega_{2}^{+}\right) \overline{b_{h}^{+}\left(r^{\prime}, \theta^{\prime}, \rho_{2}^{+}, \omega_{2}^{+}\right)} J_{2}\right)\right|_{r^{\prime}=r, \theta^{\prime}=\theta} .
\end{aligned}
$$

Define $b_{0}^{+}$by

$$
\overline{b_{0}^{+}(r, \theta, \rho, \omega)}:=\left.\left(a_{0}^{+}(r, \theta, \rho, \omega)\right)^{-1} c^{+}\left(r, \theta, \rho_{1}^{+}, \omega_{1}^{+}\right) J_{1}\right|_{r^{\prime}=r, \theta^{\prime}=\theta^{\prime}} .
$$

Since $\operatorname{supp} c^{+} \subset \Gamma_{s}^{+}(R, \varepsilon)$, Lemma 5.9 (i) implies that $\left.c^{+}\left(r, \theta, \rho_{1}^{+}, \omega_{1}^{+}\right)\right|_{r^{\prime}=r, \theta^{\prime}=\theta}$ is supported in $\Gamma_{s}^{+}(\lambda)$. Since $a_{0}^{+}$is elliptic on $\Gamma_{s}^{+}(\lambda), b_{0}^{+}$is well-defined and supported in $\Gamma_{s}^{+}(\lambda)$. Moreover, (5.18) implies that $b_{0}^{+} \in S_{\mathrm{sc}}\left(\Gamma^{+}(\lambda)\right)$. Next, let $j \geq 1$ and assume that $b_{k}^{+} \in S_{\mathrm{sc}}\left(\Gamma^{+}(\lambda)\right)$ for all $k<j$. We then define $b_{j}^{+}$by

$$
\left.a_{0}^{+}\left(r, \theta, \rho_{2}^{+}, \omega_{2}^{+}\right) \overline{b_{j}^{+}\left(r^{\prime}, \theta^{\prime}, \rho_{2}^{+}, \omega_{2}^{+}\right)} J_{2}\right|_{r^{\prime}=r, \theta^{\prime}=\theta}=r_{j}^{+}(r, \theta, \rho, \omega),
$$

where $r_{j}^{+}$takes the form

$$
\left.\sum_{\substack { k=0 \\
\begin{subarray}{c}{l+|\alpha|=k, k_{0}+k_{1}=j-k, k_{1} \leq j-1{ k = 0 \\
\begin{subarray} { c } { l + | \alpha | = k , \\
k _ { 0 } + k _ { 1 } = j - k , \\
k _ { 1 } \leq j - 1 } }\end{subarray}} \frac{1}{l ! \alpha !}\left(\partial_{\rho}^{l} \partial_{\omega}^{\alpha} D_{r^{\prime}}^{l} D_{\theta^{\prime}}^{\alpha} a_{k_{0}}^{+}\left(r, \theta, \rho_{2}^{+}, \omega_{2}^{+}\right) \overline{b_{k_{1}}^{+}\left(r^{\prime}, \theta^{\prime}, \rho_{2}^{+}, \omega_{2}^{+}\right)} J_{2}\right)\right|_{r^{\prime}=r, \theta^{\prime}=\theta} .
$$

Substituting $(\rho, \omega)=\left(\rho_{1}^{+}, \omega_{1}^{+}\right)$for $(5.22)$ and dividing by $a_{0}^{+}$, we have

$$
\overline{b_{j}^{+}(r, \theta, \rho, \omega)}=\left.\left(a_{0}^{+}\left(r^{\prime}, \theta^{\prime}, \rho, \omega\right)\right)^{-1} r_{j}^{+}\left(r, \theta, \rho_{1}^{+}, \omega_{1}^{+}\right) J_{1}\right|_{r^{\prime}=r, \theta^{\prime}=\theta^{\prime}} .
$$

By induction hypothesis, we conclude that $b_{j}^{+} \in S_{\mathrm{sc}}\left(\Gamma^{+}(\lambda)\right)$. The proof for the incoming case is similar.

5.2. Construction of the parametrix. By using the FIO defined in the previous subsection, we construct the semiclassical Isozaki-Kitada parametrix.

Theorem 5.10. For any $N \geq 0$, there exist $R_{\mathrm{IK}}, \lambda_{\mathrm{IK}}>0$ large enough and $\varepsilon_{\mathrm{IK}}>0$ small enough such that if $R \geq R_{\mathrm{IK}}, 0<\varepsilon \leq \varepsilon_{\mathrm{IK}}$ and $\lambda \geq \lambda_{\mathrm{IK}}$, then we can find

$$
b_{h}^{ \pm}=\sum_{j=0}^{N} h^{j} b_{j}^{ \pm} \quad \text { with } \quad b_{j}^{ \pm} \in S_{\mathrm{sc}}\left(\Gamma^{ \pm}\left(\lambda^{3}\right)\right), j=0,1, \ldots, N,
$$

such that, for any $a_{s}^{ \pm} \in S_{\mathrm{sc}}\left(\Gamma^{ \pm}(R, \varepsilon)\right)$, there exist

$$
c_{h}^{ \pm}=\sum_{j=0}^{N} h^{j} c_{j}^{ \pm} \quad \text { with } \quad c_{j}^{ \pm} \in S_{\mathrm{sc}}\left(\Gamma^{ \pm}(\lambda)\right), j=0,1, \ldots, N,
$$

such that, for all $T>0, h \in(0,1]$ and $0 \leq \pm t \leq T h^{-1}$,

$$
\left\|e^{-i t h \widehat{P}} \operatorname{Op}_{\kappa}\left(a_{s}^{ \pm}\right)-\kappa^{*} I_{\mathrm{IK}}^{ \pm}\left(b_{h}^{ \pm}\right) e^{-i t h \frac{1}{2} D_{r}^{2}} I_{\mathrm{IK}}^{ \pm}\left(c_{h}^{ \pm}\right)^{*} \kappa_{*}\right\|_{\mathcal{L}\left(L^{2}(\widehat{M})\right)} \leq C_{N, T} h^{N-1},
$$

where $C_{N, T}$ may be taken uniformly with respect to $h, t$ and $R$.

Remark 5.11. Since $\pi\left(\operatorname{supp} b_{h}^{ \pm}\right), \pi\left(\operatorname{supp} c_{h}^{ \pm}\right) \subset(2, \infty) \times \tilde{U}_{\kappa}$, we see that

$$
I_{\mathrm{IK}}^{ \pm}\left(b_{h}^{ \pm}\right) e^{-i t h \frac{1}{2} D_{r}^{2}} I_{\mathrm{IK}}^{ \pm}\left(c_{h}^{ \pm}\right)^{*}=\tilde{\Psi}_{\kappa} I_{\mathrm{IK}}^{ \pm}\left(b_{h}^{ \pm}\right) e^{-i t h \frac{1}{2} D_{r}^{2}} I_{\mathrm{IK}}^{ \pm}\left(c_{h}^{ \pm}\right)^{*} \tilde{\Psi}_{\kappa},
$$

and $\kappa^{*} I_{\mathrm{IK}}^{ \pm}\left(b_{h}^{ \pm}\right) e^{-i t h \frac{1}{2} D_{r}^{2}} I_{\mathrm{IK}}^{ \pm}\left(c_{h}^{ \pm}\right)^{*} \kappa^{*}$ is well-defined on $M$. 
We shall prove Theorem 5.10 for the outgoing case, and the proof for the incoming case is completely analogous. Set $B_{+}:=I_{\mathrm{IK}}^{+}\left(b_{h}^{+}\right), C_{+}:=I_{\mathrm{IK}}^{+}\left(c_{h}^{+}\right)$. By the Duhamel formula, we have

$$
\begin{aligned}
& e^{-i t h \widehat{P}^{*} B_{+} C_{+}^{*} \kappa_{*}} \\
& =\kappa^{*} B_{+} e^{-i t h \frac{1}{2} D_{r}^{2}} C_{+}^{*} \kappa_{*}-\frac{i}{h} \int_{0}^{t} e^{-i(t-s) h \widehat{P}} \kappa^{*}\left(h^{2} \widehat{P}_{\kappa} B_{+}-B_{+} h^{2} \frac{1}{2} D_{r}^{2}\right) e^{-i s h \frac{1}{2} D_{r}^{2}} C_{+}^{*} d s .
\end{aligned}
$$

To prove (5.23), it suffices to show that

$$
\begin{aligned}
&\left\|\left(h^{2} \widehat{P}_{\kappa} B_{+}-B_{+} h^{2} \frac{1}{2} D_{r}^{2}\right) e^{-i s h \frac{1}{2} D_{r}^{2}} C_{+}^{*}\right\|_{\mathcal{L}\left(L^{2}\left(\mathbb{R}^{d}\right)\right)} \lesssim h^{N+1}, \\
&\left\|a_{s}^{+}\left(r, \theta, h D_{r}, h D_{\theta}\right)-B_{+} C_{+}^{*}\right\|_{\mathcal{L}\left(L^{2}\left(\mathbb{R}^{d}\right)\right)} \lesssim h^{N},
\end{aligned}
$$

uniformly with respect to $h \in(0,1], 0 \leq s \leq T h^{-1}$ and $R>0$. To prove above two estimates, we prepare several lemmas.

Let $p+p_{1}+p_{2}$ be the full symbol of $\widehat{P}_{\kappa}$ :

$$
\widehat{P}_{\kappa}=p\left(r, \theta, D_{r}, D_{\theta}\right)+p_{1}\left(r, \theta, D_{r}, D_{\theta}\right)+p_{2}(r, \theta) .
$$

Choosing $R_{0}>0$ and $\varepsilon_{0}>0$ so that $S^{+}$is well-defined and solves the Hamilton-Jacobi equation on $\Gamma_{s}^{+}\left(R_{0}, \varepsilon_{0}\right)$, we define smooth tensors $X^{+}$and $Y^{+}$by

$$
X^{+}:=\partial_{\rho, \omega} p\left(r, \theta, \partial_{r} S^{+}, \partial_{\theta} S^{+}\right), \quad Y^{+}:=\left(p+p_{1}\right)\left(r, \theta, \partial_{r}, \partial_{\theta}\right) S^{+},
$$

and define symbols $d_{j}^{+}, j=1,2, \ldots, N+2$, by

$$
h^{2} \widehat{P}_{\kappa} B_{+}-B_{+} h^{2} \frac{1}{2} D_{r}^{2}=\sum_{j=1}^{N+2} h^{j} I_{\mathrm{IK}}^{+}\left(d_{j}^{+}\right)
$$

Then $d_{j}^{+}$should satisfy

$$
\left\{\begin{array}{l}
i d_{1}^{+}=X^{+} \cdot \partial_{r, \theta} b_{0}^{+}+Y^{+} b_{0}^{+}, \\
i d_{j}^{+}=X^{+} \cdot \partial_{r, \theta} b_{j-1}^{+}+Y^{+} b_{j-1}^{+}+i \widehat{P}_{\kappa} b_{j-2}, \quad j=2, \ldots, N+2,
\end{array}\right.
$$

where $b_{N+1}^{+} \equiv 0$. To construct $b_{j}^{+}$, we solve transport equations.

Lemma 5.12. There exist $R_{1} \geq R_{0}, \lambda_{1}>1$ large enough and $\varepsilon_{1} \leq \varepsilon_{0}$ small enough such that, for all $N \geq 0, R \geq \lambda_{1}^{N+5} R_{1}$ and $0<\varepsilon \leq \lambda_{1}^{-N-5} \varepsilon_{1}$, we can find $b_{j}^{+} \in S_{\mathrm{sc}}\left(\Gamma_{s}^{+}\left(\lambda_{1}^{3}\right)\right), j=0,1, \ldots, N$ such that $b_{0}^{+}$is elliptic on $\Gamma_{s}^{+}\left(\lambda_{1}\right)$, and that $b_{j}^{+}$solve transport equations on $\Gamma_{s}^{+}\left(\lambda_{1}^{2}\right)$ :

$$
\left\{\begin{aligned}
X^{+} \cdot \partial_{r, \theta} b_{0}^{+}+Y^{+} b_{0}^{+} & =0, \\
X^{+} \cdot \partial_{r, \theta} b_{j}^{+}+Y^{+} b_{j}^{+}+i \widehat{P}_{\kappa} b_{j-1}^{+} & =0, \quad j=1, \ldots, N .
\end{aligned}\right.
$$

For $(r, \theta, \rho, \omega) \in \Gamma_{s}^{+}\left(\lambda_{1}^{N+4}\right)$, we consider the flow $\left(r^{+}(t), \theta^{+}(t)\right)$ generated by $X^{+}$, i.e.,

$$
\left(r^{+}(t), \theta^{+}(t)\right)=\left(r^{+}, \theta^{+}\right)(t, r, \theta, \rho, \omega)
$$

is the solution to

$$
\left\{\begin{array}{l}
\left(\dot{r}^{+}(t), \dot{\theta}^{+}(t)\right)=X^{+}\left(r^{+}(t), \theta^{+}(t), \rho, \omega\right), \\
\left(r^{+}(0), \theta^{+}(0)\right)=(r, \theta) .
\end{array}\right.
$$

Then $\left(r^{+}(t), \theta^{+}(t)\right)$ is defined on $[0, \infty) \times \Gamma_{s}^{+}\left(\lambda_{1}^{N+4}\right)$, and satisfies the following:

Lemma 5.13. For all $t \geq 0,(r, \theta, \rho, \omega) \in \Gamma_{s}^{+}\left(\lambda_{1}^{N+4}\right)$ and $(j, \alpha, k, \beta) \in \mathbb{Z}_{+}^{2 d}$,

$$
\begin{aligned}
\left(r^{+}(t), \theta^{+}(t), \rho, \omega\right) & \in \Gamma_{s}^{+}\left(\lambda_{1}^{N+5}\right), \\
\left|\partial_{r}^{j} \partial_{\theta}^{\alpha} \partial_{\rho}^{k} \partial_{\omega}^{\beta}\left(r^{+}(t)-r-t \rho\right)\right| & \leq C_{j \alpha k \beta} r^{1-j-|\beta|}|\omega / r|^{(2-|\beta|)_{+}}, \\
\left|\partial_{r}^{j} \partial_{\theta}^{\alpha} \partial_{\rho}^{k} \partial_{\omega}^{\beta}\left(\theta^{+}(t)-\theta\right)\right| & \leq C_{j \alpha k \beta} r^{-j-|\beta|}|\omega / r|^{(1-|\beta|)_{+}},
\end{aligned}
$$


Proof. Let $(r, \theta, \rho, \omega) \in \Gamma_{s}^{+}\left(\lambda_{1}^{N+4}\right)$. Since $X^{+}=\left(\partial_{r} S^{+}, r^{-2}\left(h^{j k}+a^{j k}\right) \partial_{\theta^{k}} S^{+}\right)$, it follows from (5.1) that

$$
\left|\dot{r}^{+}(t)-\rho\right| \leq C_{0}\left|\omega / r^{+}(t)\right|^{2}, \quad\left|\dot{\theta}^{+}(t)\right| \leq C_{0} r^{+}(t)^{-1}\left|\omega / r^{+}(t)\right|
$$

with some $C_{0}>0$. In particular, we have

$$
\left|\dot{r}^{+}(0)-\rho\right| \leq C_{0}|\omega / r|^{2} \leq C_{1} \varepsilon_{1}, \quad\left|\dot{\theta}^{+}(0)\right| \leq C_{0} r^{-1}|\omega / r| \leq C_{1} r^{-1} \varepsilon_{1}
$$

with some $C_{1}>0$. We set $C_{2}=\inf J$ and

$$
F:=\left\{t \geq 0\left|r^{+}(s) \geq r+s \rho / 2,\right| \theta^{+}(s)-\theta\left|\leq 4 C_{0} C_{2}^{-1}\right| \omega / r \mid \text { for } 0 \leq s \leq t\right\} .
$$

By (5.30), it is easy to see that $0 \in F \neq \emptyset$. Let $t_{0}=\sup F$. We then have

$$
\left|\dot{r}^{+}(t)-\rho\right| \leq C_{0}(r+s \rho / 2)^{-2}|\omega|^{2} \leq C_{0} C_{1} \varepsilon_{1}^{2}, \quad\left|\dot{\theta}^{+}(t)\right| \leq C_{0}(r+s \rho / 2)^{-2}|\omega|,
$$

for $0 \leq t \leq t_{0}$, and hence

$$
\left|r^{+}(t)-r-t \rho\right| \leq C_{0} C_{1} \varepsilon_{1}^{2} t, \quad\left|\theta^{+}(t)-\theta\right| \leq 2 C_{0} C_{2}^{-1}|\omega / r|,
$$

for $0 \leq t \leq t_{0}$. Choosing $\varepsilon_{1}>0$ such that $\rho-C_{0} C_{1} \varepsilon_{1}^{2}>\rho / 2$ and $\delta>0$ small enough, we see that $t_{0}+\delta \in F$ which implies $t_{0}=\infty$. Therefore, $\left(r^{+}(t), \theta^{+}(t)\right)$ is well-defined on $[0, \infty)$, and satisfies (5.28) and (5.29) with $(j, \alpha, k, \beta)=0$ for all $t \geq 0$. In particular, $\left(r^{+}(t), \theta^{+}(t), \rho, \omega\right) \in \Gamma_{s}^{+}\left(\lambda_{1}^{N+5}\right)$ for $t \geq 0$, provided that $\lambda_{1}>0$ large enough. The proof for higher derivatives is obtained by (5.1) and an induction with respect to $j+|\alpha|+k+|\beta|$.

Proof of Lemma 5.12. Define a smooth real-valued function $Z^{+}$by

$$
Z^{+}(t):=Y^{+}\left(r^{+}(t), \theta^{+}(t), \rho, \omega\right) \in C^{\infty}\left([0, \infty) \times \Gamma_{s}^{+}\left(\lambda_{1}^{N+4}\right)\right) .
$$

By (2.3), (5.1) and Lemma (5.13), we have $Z^{+}(t) \in L_{t}^{1}([0, \infty))$ and

$$
\left|\partial_{r}^{j} \partial_{\theta}^{\alpha} \partial_{\rho}^{k} \partial_{\omega}^{\beta} \int_{0}^{\infty} Z^{+}(t, r, \theta, \rho, \omega) d t\right| \leq C_{j \alpha k \beta} r^{-j-|\beta|} \quad \text { on } \quad \Gamma_{s}^{+}\left(\lambda_{1}^{N+4}\right) .
$$

We now define smooth functions $\tilde{b}_{j}$ on $\Gamma_{s}^{+}\left(\lambda_{1}^{N+4-j}\right)$ by

$$
\left\{\begin{array}{l}
\tilde{b}_{0}(r, \theta, \rho, \omega)=e^{\int_{0}^{\infty} Z^{+}(t) d t} \\
\tilde{b}_{j}(r, \theta, \rho, \omega)=\int_{0}^{\infty}\left(i \widehat{P}_{\kappa} \tilde{b}_{j-1}\right)\left(r^{+}(t), \theta^{+}(t), \rho, \omega\right) e^{\int_{0}^{t} Z^{+}(s) d s} d t, \quad j=1,2, \ldots, N .
\end{array}\right.
$$

By a standard Hamilton-Jacobi theory, $\tilde{b}_{j}$ solve (5.27). Moreover, by (2.3), Lemma 5.13 and (5.31), we have

$$
\left|\partial_{r}^{l} \partial_{\theta}^{\alpha} \partial_{\rho}^{k} \partial_{\omega}^{\beta} \tilde{b}_{j}(r, \theta, \rho, \omega)\right| \leq C_{l \alpha k \beta} r^{-l-|\beta|} \quad \text { on } \quad \Gamma_{s}^{+}\left(\lambda_{1}^{N+4-j}\right) .
$$

Take $\chi^{+} \in C_{0}^{\infty}\left(\mathbb{R}^{2 d}\right)$ so that $0 \leq \chi^{+} \leq 1, \chi^{+} \equiv 1$ on $\Gamma_{s}^{+}\left(\lambda_{1}^{2}\right)$, supp $\chi^{+} \subset \Gamma_{s}^{+}\left(\lambda_{1}^{3}\right)$, and that

$$
\left|\partial_{r}^{l} \partial_{\theta}^{\alpha} \partial_{\rho}^{k} \partial_{\omega}^{\beta} \chi^{+}(r, \theta, \rho, \omega)\right| \leq C_{l \alpha k \beta} r^{-l-|\beta|} \quad \text { on } \Gamma_{s}^{+}\left(\lambda_{1}^{3}\right),
$$

and define $b_{j}^{+}:=\chi^{+} \tilde{b}_{j}$. By the construction, $b_{j}^{+}$solve (5.27) on $\Gamma_{s}^{+}\left(\lambda_{1}^{2}\right), b_{0}^{+}$is elliptic on $\Gamma_{s}^{+}\left(\lambda_{1}\right)$ and $b_{j}^{+} \in S_{\mathrm{sc}}\left(\Gamma_{s}^{+}\left(\lambda_{1}^{3}\right)\right)$.

Proof of Theorem 5.10. Let $R \geq \lambda_{1}^{N+5} R_{1}$ and $0<\varepsilon \leq \lambda_{1}^{-N-5} \varepsilon_{1}$. We first prove (5.25). By Proposition 5.8, there exists a symbol $c_{h}^{+}=\sum_{j=0}^{N} h^{j} c_{j}^{+} \in S_{\mathrm{sc}}\left(\Gamma_{s}^{+}\left(\lambda_{1}\right)\right)$ such that

$$
\left\|a_{s}^{+}\left(r, \theta, h D_{r}, h D_{\theta}\right)-B_{+} C_{+}^{*}\right\|_{\mathcal{L}\left(L^{2}\left(\mathbb{R}^{d}\right)\right)} \leq C_{N} h^{N+1}, h \in(0,1]
$$

where $C_{N}$ may be taken uniformly with respect to $h$ and $R$.

We next prove (5.24). Let $d_{j}^{+}, j=1,2, \ldots, N+2$, be defined by (5.26). Then $d_{j}^{+} \in S_{\mathrm{sc}}\left(\Gamma_{s}^{+}\left(\lambda_{1}^{3}\right)\right)$ and $d_{j}^{+} \equiv 0$ on $\Gamma_{s}^{+}\left(\lambda_{1}^{2}\right)$. For each $j$ and $k, I_{\mathrm{IK}}^{+}\left(d_{j}^{+}\right) e^{-i s h \frac{1}{2} D_{r}^{2}} I_{\mathrm{IK}}^{+}\left(c_{k}^{+}\right)^{*}$ has the distribution kernel

$$
I(s, h)=(2 \pi h)^{-d} \int e^{\frac{i}{h}\left(\left(r-r^{\prime}\right) \rho_{1}^{+}+\left(\theta-\theta^{\prime}\right) \cdot \omega_{1}^{+}-\frac{1}{2} s \rho^{2}\right)} d_{j}^{+}(r, \theta, \rho, \omega) \overline{c_{k}^{+}\left(r^{\prime}, \theta^{\prime}, \rho, \omega\right)} d \rho d \omega,
$$


where $\left(\rho_{1}^{+}, \omega_{1}^{+}\right)\left(r, \theta, \rho, \omega, r^{\prime}, \theta^{\prime}\right)$ is defined in Lemma 5.9. Since $d_{j}^{+} \equiv 0$ on $\Gamma_{s}^{+}\left(\lambda_{1}^{2}\right)$, one of the followings hold at least:

$$
\begin{aligned}
& \lambda_{1}^{-3} R<r \leq \lambda_{1}^{-2} R, \\
& \theta \in \tilde{U}_{\kappa, \sqrt{\lambda_{1}^{3} \varepsilon}} \backslash \tilde{U}_{\kappa, \sqrt{\lambda_{1}^{2}} \varepsilon}, \\
& p(r, \theta, \rho, \omega) \in J_{\lambda_{1}^{6} \varepsilon^{2}} \backslash J_{\lambda_{1}^{2} \varepsilon^{2}}, \\
& \lambda_{1}^{2} \varepsilon \lesssim|\omega / r| \lesssim \lambda_{1}^{3} \varepsilon .
\end{aligned}
$$

We first assume (5.32) or (5.35). Then, it is easy to see that

$$
r-r^{\prime}<-R /\left(2 \lambda_{1}\right)
$$

if $R>0$ is large enough. Therefore, by (5.19), we have

$$
\left(r-r^{\prime}\right) \partial_{\rho} \rho_{1}^{+}+\left(\theta-\theta^{\prime}\right) \partial_{\rho} \omega_{1}^{+}-s \rho \leq-R /\left(4 \lambda_{1}\right)-s \rho \leq-R_{0} / 2-s \rho \leq-\langle s\rangle,
$$

and we obtain (5.24) by a standard integration by parts. We next assume (5.34). Since $p(r, \theta, \rho, \omega)-\rho^{2} / 2=O\left(|\omega / r|^{2}\right)=O\left(\varepsilon_{0}^{2}\right)$, for sufficiently small $\varepsilon_{0}>0$, the amplitude vanishes identically in this case, and we have (5.24). Finally, we assume $\theta \in \tilde{U}_{\kappa, \sqrt{\lambda_{1}^{3} \varepsilon}} \backslash \tilde{U}_{\kappa, \sqrt{\lambda_{1}^{2} \varepsilon}}$. Since $\theta^{\prime} \in \tilde{U}_{\kappa, \sqrt{\lambda_{1} \varepsilon}}$ and

$$
\left|\partial_{\omega} \rho_{1}^{+}\right| \lesssim r^{-1}|\omega / r| \lesssim r^{-1} \lambda_{1}^{3} \varepsilon \lesssim r^{-1} \sqrt{\lambda_{1} \varepsilon_{1} \varepsilon}, \quad\left|\partial_{\omega}\left(\omega_{1}^{+}-\omega\right)\right| \lesssim|\omega / r| \lesssim \lambda_{1}^{3} \varepsilon \lesssim \varepsilon_{1},
$$

we have

$$
\left|\theta-\theta^{\prime}\right| \gtrsim \lambda_{1} \sqrt{\varepsilon}
$$

Therefore,

$$
\begin{aligned}
& \left|\partial_{\omega}\left(\left(r-r^{\prime}\right) \rho_{1}^{+}+\left(\theta-\theta^{\prime}\right) \cdot \omega_{1}^{+}-s \rho^{2} / 2\right)\right| \\
& \geq\left|\theta-\theta^{\prime}\right|\left(1-C \varepsilon_{1}\right)-C\left|r-r^{\prime}\right| r^{-1} \sqrt{\lambda \varepsilon_{1} \varepsilon} \\
& \gtrsim\left(\lambda_{1}-C \varepsilon_{1}-\sqrt{\lambda_{1} \varepsilon_{1}}\right) \sqrt{\varepsilon} \\
& >\sqrt{\varepsilon},
\end{aligned}
$$

for sufficiently large $\lambda_{1}>0$ and small $\varepsilon_{1}>0$. We now fix the constants $R_{\mathrm{IK}}, \varepsilon_{\mathrm{IK}}, \lambda_{\mathrm{IK}}$ so that $\lambda_{\mathrm{IK}}=\lambda_{1}, R_{\mathrm{IK}}=\lambda_{\mathrm{IK}}^{N+5} R_{1}, \varepsilon_{\mathrm{IK}}=\lambda_{\mathrm{IK}}^{-N-5} \varepsilon_{1}$. Put

$$
L\left(r, \theta, \rho, \omega, r^{\prime}, \theta^{\prime}\right)=\frac{h\left(\left(r-r^{\prime}\right) \partial_{\omega} \rho_{1}^{+}+\left(\theta-\theta^{\prime}\right) \cdot \partial_{\omega} \omega_{1}^{+}\right)}{i\left|\left(r-r^{\prime}\right) \partial_{\omega} \rho_{1}^{+}+\left(\theta-\theta^{\prime}\right) \cdot \partial_{\omega} \omega_{1}^{+}\right|^{2}} \cdot \partial_{\omega}
$$

and integrate by parts $I(s, h)$ with respect to $L$. For any $n \geq 0, I(s, h)$ then reads

$$
I(s, h)=h^{n}(2 \pi h)^{-d} \int e^{\frac{i}{h}\left(\left(r-r^{\prime}\right) \rho_{1}^{+}+\left(\theta-\theta^{\prime}\right) \cdot \omega_{1}^{+}-\frac{1}{2} s \rho^{2}\right)} G^{+}\left(r, \theta, \rho, \omega, r^{\prime}, \theta^{\prime}\right) d \rho d \omega,
$$

where $G^{+}=\left(L^{*}\right)^{n}\left(d_{j}^{+}(r, \theta, \rho, \omega) \overline{c_{k}^{+}\left(r^{\prime}, \theta^{\prime}, \rho, \omega\right)}\right)$. Using the change of the variables $(\rho, \omega) \mapsto$ $\left(\rho_{2}^{+}, \omega_{2}^{+}\right)$, we have

$$
I(s, h)=h^{n}(2 \pi h)^{-d} \int e^{\frac{i}{h} \Phi^{+}\left(s, r, \theta, \rho, \omega, r^{\prime}, \theta^{\prime}\right)} G^{+}\left(r, \theta, \rho_{+}^{2}, \omega_{+}^{2}, r^{\prime}, \theta^{\prime}\right) d \rho d \omega,
$$

where $\Phi^{+}\left(s, r, \theta, \rho, \omega, r^{\prime}, \theta^{\prime}\right):=\left(r-r^{\prime}\right) \rho+\left(\theta-\theta^{\prime}\right) \cdot \omega-\frac{1}{2} s \rho_{2}^{+}\left(r, \theta, \rho, \omega, r^{\prime}, \theta^{\prime}\right)^{2}$, and $\left(\rho_{2}^{+}, \omega_{2}^{+}\right)$is the inverse of $\left(\rho_{1}^{+}, \omega_{1}^{+}\right)$. By Lemma 5.9 and (5.36), $\rho_{2}^{+}\left(r, \theta, \rho, \omega, r^{\prime}, \theta^{\prime}\right)$ and $G^{+}\left(r, \theta, \rho_{+}^{2}, \omega_{+}^{2}, r^{\prime}, \theta^{\prime}\right)$ are smooth and uniformly bounded functions on $\mathbb{R}^{3 d}$. Applying the Calderón-Vaillancourt theorem, we hence have

$$
\left\|I_{\mathrm{IK}}^{+}\left(d_{j}^{+}\right) e^{-i s h \frac{1}{2} D_{r}^{2}} I_{\mathrm{IK}}^{+}\left(c_{k}^{+}\right)^{*}\right\|_{\mathcal{L}\left(L^{2}\left(\mathbb{R}^{d}\right)\right)} \leq C_{N, d, \varepsilon} h^{n-n_{d}}\langle s\rangle^{n_{d}} \leq C_{N, d, \varepsilon, T} h^{n-2 n_{d}}
$$

for all $n \geq 0, h \in(0,1]$ and $0 \leq s \leq T h^{-1}$, where $n_{d}>0$ depends only on $d$, and $C_{N, d, \varepsilon, T}$ is independent of $h$ and $R$. Choosing $n>0$ with $n-2 n_{d}>N+1$, we complete the proof 
5.3. Dispersive estimates. We here prove dispersive estimates for the Isozaki-Kitada parametrix. Let $R_{\mathrm{IK}}, \varepsilon_{\mathrm{IK}}, \lambda_{\mathrm{IK}}>0$ be as in Theorem 5.10. In this subsection we use the notation $\partial^{\gamma}:=$ $\partial_{r}^{j} \partial_{\theta}^{\alpha} \partial_{\rho}^{k} \partial_{\omega}^{\beta} \partial_{r^{\prime}}^{j^{\prime}} \partial_{\theta^{\prime}}^{\alpha^{\prime}}$ for $\gamma=\left(j, \alpha, k, \beta, j^{\prime}, \alpha^{\prime}\right) \in \mathbb{Z}_{+}^{3 d}$.

Theorem 5.14. For sufficiently large $R>R_{\mathrm{IK}}$, small $0<\varepsilon<\varepsilon_{\mathrm{IK}}$, all $b^{ \pm} \in S_{\mathrm{sc}}\left(\Gamma^{ \pm}\left(\lambda_{\mathrm{IK}}^{3}\right)\right)$ and $c^{ \pm} \in S_{\mathrm{sc}}\left(\Gamma^{ \pm}\left(\lambda_{\mathrm{IK}}\right)\right)$, we can write

$$
I_{\mathrm{IK}}^{ \pm}\left(b^{ \pm}\right) e^{-i t h \frac{1}{2} D_{r}^{2}} I_{\mathrm{IK}}^{ \pm}\left(c^{ \pm}\right)^{*}=U_{\mathrm{IK}}^{ \pm}(t, h)+R_{\mathrm{IK}}^{ \pm}(t, h),
$$

where $U_{\mathrm{IK}}^{ \pm}(t, h)$ satisfy dispersive estimates

$$
\left\|r^{-\frac{d-1}{2}} U_{\mathrm{IK}}^{ \pm}(t, h) r^{-\frac{d-1}{2}}\right\|_{\mathcal{L}\left(L^{1}\left(\mathbb{R}^{d}\right), L^{\infty}\left(\mathbb{R}^{d}\right)\right)} \leq C|t h|^{-d / 2}, \quad 0< \pm t \leq h^{-1}, h \in(0.1],
$$

and $R_{\mathrm{IK}}^{ \pm}(t, h)$ are rapidly decaying with respect to $h$ : for any $N \geq 0$,

$$
\left\|R_{\mathrm{IK}}^{ \pm}(t, h)\right\|_{\mathcal{L}\left(L^{2}\left(\mathbb{R}^{d}\right)\right)} \leq C_{N} h^{N}, \quad 0< \pm t \leq h^{-1}, h \in(0.1] .
$$

Moreover $C, C_{N}>0$ can taken uniformly with respect to $h, t$ and $R$.

We prove the theorem for the case $t \geq 0$, and the proof for the case $t \leq 0$ is similar. The distribution kernel of $I_{\mathrm{IK}}^{+}\left(b^{+}\right) e^{-i t h \frac{1}{2} D_{r}^{2}} I_{\mathrm{IK}}^{+}\left(c^{+}\right)^{*}$ takes the form

$$
I_{A^{+}}(t, h)=(2 \pi h)^{-d} \int e^{\frac{i}{h} \Phi^{+}\left(t, r, \theta, \rho, \omega, r^{\prime}, \theta^{\prime}\right)} A^{+}\left(r, \theta, \rho, \omega, r^{\prime}, \theta^{\prime}\right) d \rho d \omega,
$$

where

$$
\begin{aligned}
\Phi^{+}\left(t, r, \theta, \rho, \omega, r^{\prime}, \theta^{\prime}\right) & :=\left(r-r^{\prime}\right) \rho+\left(\theta-\theta^{\prime}\right) \cdot \omega-\frac{1}{2} t\left(\rho_{2}^{+}\right)^{2}, \\
A^{+}\left(r, \theta, \rho, \omega, r^{\prime}, \theta^{\prime}\right) & :=b^{+}\left(r, \theta, \rho_{2}^{+}, \omega_{2}^{+}\right) \overline{b^{+}\left(r^{\prime}, \theta^{\prime}, \rho_{2}^{+}, \omega_{2}^{+}\right)}\left|\operatorname{det} \partial_{\rho, \omega}\left(\rho_{2}^{+}, \omega_{2}^{+}\right)\right|,
\end{aligned}
$$

and $\left(\rho_{2}^{+}, \omega_{2}^{+}\right)=\left(\rho_{2}^{+}, \omega_{2}^{+}\right)\left(r, \theta, \rho, \omega, r^{\prime}, \theta^{\prime}\right)$ is given by Lemma 5.9. Note that since $\left(r, \theta, \rho_{2}^{+}, \omega_{2}^{+}\right) \in$ $\Gamma_{s}^{+}\left(\lambda_{\mathrm{IK}}^{3}\right)$ and $\left(r^{\prime}, \theta^{\prime}, \rho_{2}^{+}, \omega_{2}^{+}\right) \in \Gamma_{s}^{+}\left(\lambda_{\mathrm{IK}}\right),(5.17)$ implies

$$
C^{-1} \leq \rho \leq C, \quad\left|\omega / \sqrt{r r^{\prime}}\right| \leq C \varepsilon_{1} \quad \text { on } \quad \operatorname{supp} A^{+},
$$

and $\partial^{\gamma} A^{+}$and $\partial^{\gamma} \rho_{2}^{+}$are uniformly bounded on $\mathbb{R}^{3 d}$.

We first remove a smoothing term from $I_{A^{+}}(t, h)$. Let $\chi_{\rho} \in C_{0}^{\infty}(\mathbb{R}), \chi_{\omega} \in C_{0}^{\infty}\left(\mathbb{R}^{d-1}\right)$ be smooth cut-off functions such that

$$
\begin{aligned}
& \operatorname{supp} \chi_{\rho} \subset(-1,1), \quad \chi_{\rho} \equiv 1 \text { on }(-1 / 2,1 / 2), \\
& \operatorname{supp} \chi_{\omega} \subset\{|\theta| \leq 1\}, \quad \chi_{\omega} \equiv 1 \text { on }\{|\theta| \leq 1 / 2\},
\end{aligned}
$$

and define $A_{\delta}^{+}:=\chi_{\rho}\left(\partial_{\rho} \Phi^{+}\right) \chi_{\omega}\left(\partial_{\omega} \Phi^{+} / \delta\right) A^{+}$, where $\delta>0$ is a small parameter. We denote the operator having the Schwartz kernel $I_{A_{\delta}^{+}}(t, h)$ by $U_{A_{\delta}^{+}}(t, h)$.

Lemma 5.15. For all $N>0$ and $\delta>0$, we have

$$
\left\|I_{\mathrm{IK}}^{+}\left(b^{+}\right) e^{-i t h \frac{1}{2} D_{r}^{2}} I_{\mathrm{IK}}^{+}\left(c^{+}\right)^{*}-U_{A_{\delta}^{+}}(t, h)\right\|_{\mathcal{L}\left(L^{2}\left(\mathbb{R}^{d}\right)\right)} \leq C_{N} h^{N},
$$

uniformly with respect to $h \in(0,1], 0 \leq \pm t \leq h^{-1}$ and $R>0$.

Proof. We split $A^{+}-A_{\delta}^{+}=\left(1-\chi_{\rho}\right) \chi_{\omega} A^{+}-\left(1-\chi_{\omega}\right) A^{+}=: A_{1}^{+}+A_{2}^{+}$, and denote by $I_{A_{j}^{+}}$the oscillatory integral of the form (5.37) with the phase $\Phi^{+}$and the amplitude $A_{j}^{+}$, respectively. We set $L_{1}:=\left(h / i \partial_{\rho} \Phi^{+}\right) \partial_{\rho}, L_{2}:=\left(h / i\left|\partial_{\omega} \Phi^{+}\right|^{2}\right)\left(\partial_{\omega} \Phi^{+}\right) \cdot \partial_{\omega}$. Since

$$
\left|\partial^{\gamma}\left(1 / \partial_{\rho} \Phi^{+}\right)\right| \lesssim 1 \quad \text { on } \quad \operatorname{supp} A_{1}^{+}, \quad\left|\partial^{\gamma}\left(\partial_{\omega} \Phi^{+} /\left|\partial_{\omega} \Phi^{+}\right|^{2}\right)\right| \lesssim 1 / \delta \quad \text { on } \quad \operatorname{supp} A_{2}^{+},
$$

$n$-times integration by parts $I_{A_{j}^{+}}$with $L_{j}$, respectively, implies that $I_{A_{j}^{+}}$reads

$$
I_{A_{j}^{+}}=(2 \pi h)^{-d} \int e^{\frac{i}{h}\left(\left(r-r^{\prime}\right) \rho+\left(\theta-\theta^{\prime}\right) \cdot \omega\right)} \tilde{A}_{j}^{+}\left(r, \theta, \rho, \omega, r^{\prime}, \theta^{\prime}\right) d \rho d \omega,
$$


where $\tilde{A}_{j}^{+}=e^{-\frac{i}{h} \frac{t}{2}\left(\rho_{2}^{+}\right)^{2}}\left(L_{j}^{*}\right)^{n} A_{j}$ satisfies

$$
\left|\partial^{\gamma} \tilde{A}_{j}^{+}\left(r, \theta, \rho, \omega, r^{\prime}, \theta^{\prime}\right)\right| \leq C_{n} h^{n-|\gamma|}\langle t\rangle|\gamma| \leq C_{n} h^{n-2|\gamma|} \quad \text { on } \quad \mathbb{R}^{3 d},
$$

for all $\gamma \in \mathbb{Z}_{+}^{3 d}, h \in(0,1]$, and $0 \leq \pm t \leq h^{-1}$. By the Calderón-Vaillancourt theorem, there exists an integer $N_{d}>0$ depending only on $d$ such that

$$
\left\|I_{\mathrm{IK}}^{+}\left(b^{+}\right) e^{-i t h \frac{1}{2} D_{r}^{2}} I_{\mathrm{IK}}^{+}\left(c^{+}\right)^{*}-U_{A_{\delta}^{+}}(t, h)\right\|_{\mathcal{L}\left(L^{2}\left(\mathbb{R}^{d}\right)\right)} \leq C_{n} h^{n-2 N_{d}} .
$$

Choosing $n \geq 0$ with $n-2 N_{d} \geq N$, we obtain the assertion.

To prove dispersive estimates for $U_{A_{\delta}^{+}}(t, h)$, we next study the phase function more precisely

Lemma 5.16. On $\operatorname{supp} A^{+}, \frac{1}{2} \rho_{2}^{+}\left(r, \theta, \rho, \omega, r^{\prime}, \theta^{\prime}\right)^{2}$ takes the form

$$
\frac{1}{2} \rho_{2}^{+}\left(r, \theta, \rho, \omega, r^{\prime}, \theta^{\prime}\right)^{2}=\frac{1}{2} \rho^{2}+\frac{1}{2 r r^{\prime}} q_{0}(\theta, \omega)+Q^{+}\left(r, \theta, \rho, \omega, r^{\prime}, \theta^{\prime}\right),
$$

where $q_{0}(\theta, \omega)=h^{j k}(\theta) \omega_{j} \omega_{k}$. Moreover we can write

$$
Q^{+}=Q_{1}^{+}+\left(\theta-\theta^{\prime}\right) \cdot Q_{2}^{+}
$$

such that, for all $\left(r, \theta, \rho, \omega, r^{\prime}, \theta\right) \in \operatorname{supp} A^{+}$and $\gamma=\left(j, \alpha, k, \beta, j^{\prime}, \alpha^{\prime}\right) \in \mathbb{Z}_{+}^{3 d}$,

$$
\left\{\begin{array}{l}
\left|\partial^{\gamma} Q_{1}^{+}\left(r, \theta, \rho, \omega, r^{\prime}, \theta^{\prime}\right)\right| \leq C R_{1}^{-j-j^{\prime}-|\beta|}\left(\varepsilon_{1}^{(3-|\beta|)_{+}}+R_{1}^{-\mu} \varepsilon_{1}^{(2-|\beta|)_{+}}\right), \\
\left|\partial^{\gamma} Q_{2}^{+}\left(r, \theta, \rho, \omega, r^{\prime}, \theta^{\prime}\right)\right| \leq C R_{1}^{-j-j^{\prime}-|\beta|} \varepsilon_{1}^{(2-|\beta|)_{+}},
\end{array}\right.
$$

where $R_{1}, \varepsilon_{1}$ are given by Lemma 5.12.

Proof. We start from the formula $\rho_{2}^{+}=\rho-\int_{0}^{1}\left(\partial_{r} \varphi^{+}\right)\left(r_{s}, \theta_{s}, \rho_{2}^{+}, \omega_{2}^{+}\right) d s$, where $\left(r_{s}, \theta_{s}\right)=\left(r^{\prime}, \theta^{\prime}\right)+$ $s\left(r-r^{\prime}, \theta-\theta^{\prime}\right)$. By using the mean value theorem, we have

$$
\left(\partial_{r} \varphi^{+}\right)\left(r_{s}, \theta_{s}, \rho_{2}^{+}, \omega_{2}^{+}\right)=\left(\partial_{r} \varphi^{+}\right)\left(r_{s}, \theta, \rho_{2}^{+}, \omega_{2}^{+}\right)-\left(\theta-\theta^{\prime}\right) \cdot F^{+}(s),
$$

where $F^{+}(s)$ is defined by

$$
F^{+}(s):=(1-s) \int_{0}^{1}\left(\partial_{\theta} \partial_{r} \varphi^{+}\right)\left(r_{s}, \theta_{s \sigma}, \rho_{2}^{+}, \omega_{2}^{+}\right) d \sigma, \quad \theta_{s \sigma}=\theta+\sigma\left(\theta_{s}-\theta\right) .
$$

By (5.1) and (5.17), we obtain

$$
\sup _{s \in[0,1]}\left|\partial^{\gamma} F^{+}(s)\right| \lesssim R_{1}^{-j-j^{\prime}-|\beta|} \varepsilon_{1}^{(2-|\beta|)_{+}} .
$$

Since $R_{\mathrm{IK}} \geq \lambda_{\mathrm{IK}}^{4} R_{1}$ and $\varepsilon_{\mathrm{IK}} \leq \lambda_{\mathrm{IK}}^{-4} \varepsilon_{1}$, by the mean value theorem, we can write

$$
\left(\partial_{r} \varphi^{+}\right)\left(r_{s}, \theta, \rho_{2}^{+}, \omega_{2}^{+}\right)=-\frac{1}{2 r_{s}^{2} \rho} q_{0}(\theta, \omega)-G^{+}(s),
$$

where, by (5.2) and (5.17), $G^{+}(s)$ satisfies

$$
\sup _{s \in[0,1]}\left|\partial^{\gamma} G^{+}(s)\right| \lesssim R_{1}^{-j-j^{\prime}-|\beta|}\left(\varepsilon_{1}^{(3-|\beta|)_{+}}+R_{1}^{-\mu} \varepsilon_{1}^{(2-|\beta|)_{+}}\right) .
$$

Set $\tilde{F}^{+}=\int_{0}^{1} F^{+}(s) d s$ and $\tilde{G}^{+}=\int_{0}^{1} G^{+}(s) d s$. Since $\int_{0}^{1} r_{s}^{-2} d s=1 / \sqrt{r r^{\prime}}$, we have

$$
\left(\rho_{2}^{+}\right)^{2}=\left(\rho+\frac{1}{2 r r^{\prime} \rho} q_{0}(\theta, \omega)+\left(\theta-\theta^{\prime}\right) \cdot \tilde{F}^{+}+\tilde{G}^{+}\right)^{2} .
$$

If we set

$$
Q_{1}^{+}=4 \rho \tilde{G}^{+}+2\left(\frac{1}{2 r r^{\prime} \rho} q_{0}(\theta, \omega)+\left(\theta-\theta^{\prime}\right) \cdot \tilde{F}^{+}+\tilde{G}^{+}\right)^{2}, \quad Q_{2}^{+}=4 \rho \tilde{F}^{+},
$$

then $Q^{+}=Q_{1}^{+}+Q_{2}^{+}$satisfy (5.38). 
Using the change of the variable $\omega \mapsto \sqrt{r r^{\prime}} \nu, I_{A_{\delta}^{+}}(t, h)$ reads

$$
I_{A_{\delta}^{+}}(t, h)=\frac{\left(r r^{\prime}\right)^{\frac{d-1}{2}}}{(2 \pi h)^{d}} \int e^{\frac{i}{h} \Phi^{+}\left(t, r, \theta, \rho, \sqrt{r r^{\prime}} \nu, r^{\prime}, \theta^{\prime}\right)} A_{\delta}^{+}\left(r, \theta, \rho, \sqrt{r r^{\prime}} \nu, r^{\prime}, \theta^{\prime}\right) d \rho d \nu,
$$

where $\tilde{A}_{\delta}^{+}\left(r, \theta, \rho, \nu, r^{\prime}, \theta^{\prime}\right):=A_{\delta}^{+}\left(r, \theta, \rho, \sqrt{r r^{\prime}} \nu, r^{\prime}, \theta^{\prime}\right)$ is bounded on $\mathbb{R}^{3 d}$ and compactly supported with respect to $(\rho, \nu)$. Moreover, $\pi_{\rho, \nu} \circ \operatorname{supp} \tilde{A}_{\delta}^{+}$is bounded uniformly with respect to $R>0$, where $\pi_{\rho, \nu}: \mathbb{R}^{3 d} \rightarrow \mathbb{R}^{d}$ is a canonical projection onto the $(\rho, \nu)$-space.

Proposition 5.17. There exists $\delta>0$ small enough such that, for all $h \in(0,1]$ and $0<t \leq h^{-1}$, we have

$$
\left\|r^{-\frac{d-1}{2}} U_{A_{\delta}^{+}}(t, h) r^{-\frac{d-1}{2}}\right\|_{\mathcal{L}\left(L^{1}\left(\mathbb{R}^{d}\right), L^{\infty}\left(\mathbb{R}^{d}\right)\right.} \leq C|t h|^{-d / 2},
$$

where $C$ may be taken uniformly with respect to $R, h$ and $t$.

Proof. For $0<t \leq h$, it follows from

$$
r^{-\frac{d-1}{2}}\left|I_{A_{\delta}^{+}}(t, h)\right| r^{-\frac{d-1}{2}} \leq C h^{-d} \leq C|t h|^{-d / 2} .
$$

Suppose that $h \leq t \leq h^{-1}$ and assume $2 h \leq h^{-1}$ without loss of generality. Define

$$
\begin{aligned}
\tilde{\Phi}^{+}\left(t, r, \theta, \rho, \nu, r^{\prime}, \theta^{\prime}\right) & :=\frac{1}{t} \Phi^{+}\left(t, r, \theta, \rho, \sqrt{r r^{\prime}} \nu, r^{\prime}, \theta^{\prime}\right) \\
& =\frac{r-r^{\prime}}{t} \rho+\frac{\theta-\theta^{\prime}}{t} \cdot \sqrt{r r^{\prime}} \nu-\frac{1}{2} \rho_{2}^{+}\left(r, \theta, \rho, \sqrt{r r^{\prime}} \nu, r^{\prime}, \theta^{\prime}\right)^{2} .
\end{aligned}
$$

By Lemma 5.16, we obtain

$$
\partial_{\rho} \tilde{\Phi}^{+}(t)=\frac{r-r^{\prime}}{t}-\rho+O\left(\varepsilon_{1}^{2}\right), \quad \partial_{\nu} \tilde{\Phi}^{+}(t)=\frac{\sqrt{r r^{\prime}}\left(\theta-\theta^{\prime}\right)}{t}+O\left(\varepsilon_{1}\right)
$$

on the support of $\tilde{A}_{\delta}^{+}$. Thus, if we restrict the support of $\tilde{A}_{\delta}^{+}$to one of the regions

$$
\left|\frac{r-r^{\prime}}{t}-\rho\right| \geq \varepsilon_{1} \quad \text { or } \quad\left|\frac{\sqrt{r r^{\prime}}\left(\theta-\theta^{\prime}\right)}{t}\right| \geq 1,
$$

then we have $\left|\partial_{\rho} \tilde{\Phi}(t)\right|+\left|\partial_{\nu} \tilde{\Phi}(t)\right| \geq \varepsilon_{1}$ with sufficiently small $\varepsilon_{1}>0$. Take $\chi_{1} \in C_{0}^{\infty}(\mathbb{R})$ and $\chi_{2} \in C_{0}^{\infty}\left(\mathbb{R}^{d-1}\right)$ satisfying $\chi_{1} \equiv 1$ on $(-1 / 2,1 / 2)$, supp $\chi_{1} \subset(-1,1), \chi_{2} \equiv 1$ on $\{|\theta| \leq 1 / 2\}$ and $\operatorname{supp} \chi_{2} \subset\{|\theta| \leq 1\}$, and put

$$
\tilde{\chi}_{1}\left(t, r, \theta, \rho, r^{\prime}, \theta^{\prime}\right)=\chi_{1}\left(\frac{r-r^{\prime}}{\varepsilon_{1} t}-\frac{\rho}{\varepsilon_{1}}\right) \chi_{2}\left(\frac{\sqrt{r r^{\prime}}\left(\theta-\theta^{\prime}\right)}{t}\right) .
$$

Integrating by parts, we then obtain the non stationary estimates

$$
\left|(2 \pi h)^{-d} \int e^{\frac{i}{h} t \tilde{\Phi}^{+}(t)}\left(1-\tilde{\chi}_{1}\right) \tilde{A}_{\delta}^{+} d \rho d \nu\right| \leq C_{\varepsilon_{1}, \lambda_{\mathrm{IK}}} h^{-d}|t / h|^{-n} \leq C_{\varepsilon_{1}, \lambda_{\mathrm{IK}}}|t h|^{-d / 2},
$$

for all $h \in(0,1], h \leq t \leq 2 h^{-1}, \delta>0$ and $n \geq d / 2$, where $C_{\varepsilon_{1}, \lambda_{\text {IK }}}$ may be taken uniformly in $R$. Since $C^{-1} \leq \rho \leq C$ for some $C>0$, if $\varepsilon_{1}>0$ is sufficiently small, then

$$
C_{0}^{-1}|t| \leq r-r^{\prime} \leq C_{0}|t| \quad \text { with some } C_{0}>0,
$$

on the support of $\tilde{\chi}_{1} \tilde{A}_{\delta}^{+}$, and this estimate implies $\left|t / \sqrt{r r^{\prime}}\right| \lesssim 1$. We now fix $\delta$ with $0<\delta \leq \varepsilon_{1}$. Since $\partial_{\nu} \tilde{\Phi}^{+}(t)=\left(\sqrt{r r^{\prime}} / t\right) \partial_{\omega} \Phi_{+}(t)$, we have

$$
\left|\theta-\theta^{\prime}\right| \leq C\left|t / \sqrt{r r^{\prime}}\right|\left(\left|\partial_{\nu} \tilde{\Phi}^{+}(t)\right|+\varepsilon_{1}\right) \leq C_{1} \varepsilon_{1} \quad \text { with some } C_{1}>0,
$$

on the support of $\tilde{\chi}_{1} \tilde{A}_{\delta}^{+}$. We fix $\lambda_{\mathrm{IK}}^{\prime}>\lambda_{\mathrm{IK}}$ with $2 \lambda_{\mathrm{IK}}>\lambda_{\mathrm{IK}}^{\prime}$, and choose $\chi_{3}, \chi_{4} \in C_{0}^{\infty}\left(\mathbb{R}^{2 d}\right)$ so that $\chi_{3} \equiv 1$ on $\Omega^{+}\left(\lambda_{\mathrm{IK}}^{3}\right), \operatorname{supp} \chi_{3} \subset \Omega^{+}\left(\lambda_{\mathrm{IK}}^{\prime 3}\right), \chi_{4} \equiv 1$ on $\Omega^{+}\left(\lambda_{\mathrm{IK}}\right), \operatorname{supp} \chi_{4} \subset \Omega^{+}\left(\lambda_{\mathrm{IK}}^{\prime}\right)$ and

$$
\left|\partial_{r}^{j} \partial_{\theta}^{\alpha} \partial_{\rho}^{k} \partial_{\omega}^{\beta} \chi_{j}(r, \theta, \rho, \omega)\right| \leq C_{j \alpha k \beta \lambda_{1}}\langle r\rangle^{-j-|\beta|} \quad \text { on } \quad \mathbb{R}^{3 d}, \quad j=3,4 .
$$


We also choose $\chi_{5} \in C_{0}^{\infty}\left(\mathbb{R}^{d-1}\right)$ with $\chi_{5} \equiv 1$ on $\{|\theta| \leq 1 / 2\}$ and supp $\chi_{5} \subset\{|\theta| \leq 1\}$. We now define

$$
\begin{aligned}
& \Phi_{0}^{+}\left(t, r, \theta, \rho, \nu, r^{\prime}, \theta^{\prime}\right) \\
& :=\frac{r-r^{\prime}}{t} \rho+\frac{\theta-\theta^{\prime}}{t} \cdot \sqrt{r r^{\prime}} \nu-\frac{1}{2} \rho^{2}-\frac{1}{2} q_{0}(\theta, \nu)-\tilde{\chi}_{3} \tilde{\chi}_{4} \tilde{\chi}_{5} Q^{+}\left(r, \theta, \rho, \sqrt{r r^{\prime}} \nu, r^{\prime}, \theta^{\prime}\right),
\end{aligned}
$$

where $\tilde{\chi}_{3}, \tilde{\chi}_{4}$ and $\tilde{\chi}_{5}$ are defined by

$$
\begin{aligned}
\tilde{\chi}_{3}\left(r, \theta, \rho, \nu, r^{\prime}, \theta^{\prime}\right) & :=\chi_{3}\left(r, \theta,\left(\rho_{2}^{+}, \omega_{2}^{+}\right)\left(r, \theta, \rho, \sqrt{r r^{\prime}} \nu, r^{\prime}, \theta^{\prime}\right)\right) \\
\tilde{\chi}_{4}\left(r, \theta, \rho, \nu, r^{\prime}, \theta^{\prime}\right) & :=\chi_{3}\left(r^{\prime}, \theta^{\prime},\left(\rho_{2}^{+}, \omega_{2}^{+}\right)\left(r, \theta, \rho, \sqrt{r r^{\prime}} \nu, r^{\prime}, \theta^{\prime}\right)\right) \\
\tilde{\chi}_{5}\left(\theta, \theta^{\prime}\right) & :=\chi_{5}\left(\frac{\theta-\theta^{\prime}}{2 C_{1} \varepsilon_{1}}\right) .
\end{aligned}
$$

It is easy to see that $\Phi_{0}^{+}$is smooth on $[h, \infty) \times \mathbb{R}^{3 d}$ and $\Phi_{0}^{+} \equiv \tilde{\Phi}^{+}$on $\operatorname{supp} \tilde{\chi}_{1} \tilde{A}_{\delta}^{+}$. Moreover by Lemma 5.16, (5.39) and (5.40), we have

$$
\left|\partial_{\rho}^{k} \partial_{\nu}^{\beta} \Phi_{0}^{+}\left(t, r, \theta, \rho, \nu, r^{\prime}, \theta^{\prime}\right)\right| \leq C_{k \beta}
$$

for all $\left(r, \theta, \rho, \nu, r^{\prime}, \theta^{\prime}\right) \in \mathbb{R}^{3 d}, h \leq t \leq h^{-1}$ and $|k+\beta| \geq 2$, where $C_{k \beta}>0$ may be taken uniformly in $h, t$ and $R$. We also obtain

$$
\partial_{\rho, \nu}^{2} \Phi_{0}^{+}=-\left(\begin{array}{cc}
1 & 0 \\
0 & h^{j k}
\end{array}\right)+O\left(\varepsilon_{1}+R_{1}^{-\mu}\right) \quad \text { on }\left[h, h^{-1}\right] \times \mathbb{R}^{3 d} .
$$

Since $\left|\operatorname{det}\left(h^{j k}\right)\right| \gtrsim 1$, if $R_{1}$ is large enough and $\varepsilon_{1}$ is small enough,

$$
\left|\operatorname{det} \partial_{\rho, \nu}^{2} \Phi_{0}^{+}\right| \gtrsim 1
$$

on $\left[h, h^{-1}\right] \times \mathbb{R}^{3 d}$ uniformly with respect to $R, h$ and $t$. Therefore the mapping

$$
(\rho, \nu) \mapsto \partial_{\rho, \nu} \Phi_{0}^{+}\left(t, r, \theta, \rho, \nu, r^{\prime}, \theta^{\prime}\right)
$$

is a diffeomorphism from $\mathbb{R}^{d}$ to $\mathbb{R}^{d}$, and $\Phi_{0}^{+}$has a unique non-degenerate critical point $\left(\rho_{c}, \nu_{c}\right)=$ $\left(\rho_{c}, \nu_{c}\right)\left(t, r, r^{\prime}, \theta, \theta^{\prime}\right)$. Moreover, $\partial_{\rho}^{k} \partial_{\nu}^{\beta} \Phi_{0}^{+}\left(t, r, \theta, \rho_{c}, \nu_{c}, r^{\prime}, \theta^{\prime}\right)$ are bounded uniformly with respect to $R$ and $t$ if $|k+\beta| \geq 2$. We hence can apply the stationary phase theorem and obtain

$$
\begin{aligned}
\left|(2 \pi h)^{-d} \int e^{\frac{i}{h} t \tilde{\Phi}^{+}(t)} \tilde{\chi}_{1} \tilde{A}_{\delta}^{+} d \rho d \nu\right| & =\left|(2 \pi h)^{-d} \int e^{\frac{i}{h} t \Phi_{0}^{+}(t)} \tilde{\chi}_{1} \tilde{A}_{\delta}^{+} d \rho d \nu\right| \\
& \leq C_{\varepsilon_{1}, \lambda_{\mathrm{IK}}}^{\prime} h^{-d}|t / h|^{-d / 2} \\
& \leq C_{\varepsilon_{1}, \lambda_{\mathrm{IK}}}^{\prime}|t h|^{-d / 2},
\end{aligned}
$$

for $h \in(0,1]$ and $h \leq t \leq h^{-1}$, where $C_{\varepsilon_{1}, \lambda_{\mathrm{IK}}}^{\prime}>0$ does not depend on $R>0$. We complete the proof

Proof of Theorem 5.14. We set

$$
U_{\mathrm{IK}}^{+}(t, h)=U_{A_{\delta}^{+}}(t, h), \quad R_{\mathrm{IK}}^{+}(t, h)=I_{\mathrm{IK}}^{+}\left(b^{+}\right) e^{-i t h \frac{1}{2} D_{r}^{2}} I_{\mathrm{IK}}^{+}\left(c^{+}\right)^{*}-U_{A_{\delta}^{+}}(t, h) .
$$

Clearly, they satisfy the assertion. When $-h^{-1} \leq t<0$, the proof is analogous.

\section{Microlocal SMoothing Properties}

Fix arbitrarily a coordinate chart $\kappa_{0}: V_{\kappa_{0}} \rightarrow U_{\kappa_{0}}$. Let $\tilde{U}_{\kappa_{0}} \Subset U_{\kappa_{0}}, J \Subset(0, \infty)$ and $-1<\sigma<1$ be an in Definition 2.5. In this section we prove the following:

Theorem 6.1. Fix arbitrarily $t_{1}>0$ and let $\varepsilon>0$ be small enough. Then there exist $\delta_{\varepsilon, t_{1}}>0$ and $L_{\varepsilon, t_{1}}>0$ such that for all $\left(\sigma_{l}\right)_{0 \leq l \leq L_{\varepsilon, t_{1}}} \subset(-1,1 / 2]$ satisfying $(2.12)$, sufficiently large $R_{0}>0$, all $R_{2} \geq R_{1} \geq R_{0}$, all symbols

$$
a_{l}^{ \pm} \in S_{\mathrm{sc}}\left(\Gamma_{i}^{ \pm}\left(R_{1}, \tilde{U}_{\kappa_{0}}, J, \varepsilon, \delta_{\varepsilon, t_{1}}, l\right)\right) \quad b_{l}^{ \pm} \in S_{\mathrm{sc}}\left(\Omega_{i}^{ \pm}\left(R_{2}, \tilde{U}_{\kappa_{0}}, J, \varepsilon, \delta_{\varepsilon, t_{1}}, l\right)\right),
$$


and $N \geq 0$, we have

$$
\left\|\mathrm{Op}_{\kappa_{0}, h}\left(a_{l}^{ \pm}\right) e^{-i t h \widehat{P}} \operatorname{Op}_{\kappa_{0}, h}\left(b_{l}^{ \pm}\right)\right\|_{\mathcal{L}\left(L^{2}(\widehat{M})\right)} \leq C_{N, l} h^{N},
$$

uniformly with respect to $h \in(0,1], R_{2} t_{1} \leq \pm t \leq h^{-1}$ and $R_{2}$.

We prove Theorem 6.1 for the case $t \geq 0$, and the proof for case $t \leq 0$ is analogous. We need the following Egorov theorem.

Theorem 6.2 (The Egorov theorem). For any $T>0, N \geq 0$ and symbol $b^{+} \in S_{\mathrm{sc}}\left(\Omega^{+}\left(R_{2}, \tilde{U}_{\kappa_{0}}, J, \sigma\right)\right)$, there exist symbols

$$
b_{\kappa, h}^{+}(t)=\sum_{j=0}^{N} h^{j} b_{\kappa, j}^{+}(t) \quad \text { with } \quad b_{\kappa, j}^{+}(t) \in S_{\mathrm{sc}}\left(\kappa_{*} \exp t H_{p}\left(\kappa_{0 *}^{-1} \operatorname{supp} b^{+}\right)\right),
$$

and a constant $C_{N, T}>0$, independent of $R_{2}$, such that

$$
\left\|e^{-i t h \widehat{P}} \mathrm{Op}_{\kappa_{0}}\left(b^{+}\right) e^{i t h \widehat{P}}-\sum_{\kappa} \mathrm{Op}_{\kappa}\left(b_{\kappa, h}^{+}(t)\right)\right\|_{\mathcal{L}\left(L^{2}(\widehat{M})\right)} \leq C_{N, T} h^{N+1}|t|
$$

uniformly with respect to $0 \leq t \leq R_{2} T$ and $h \in(0,1]$. Moreover, $b_{\kappa, j}^{+}(t)$ is uniformly bounded in $S_{\mathrm{sc}}\left(\kappa_{*} \exp t H_{p}\left(\kappa_{0 *}^{-1} \operatorname{supp} b^{+}\right)\right)$with respect to $0 \leq t \leq R_{2} T$.

Proof. This theorem is basically well known, and we hence give the sketch of the proof. By (4.19), we can choose $t_{1}>0$, independent of $R_{2}$, such that the geodesic is contained one fixed coordinate neighborhood if $0 \leq t \leq R_{2} t_{1}$. Define the map $\tilde{\varphi}(t)=(\tilde{r}(t), \tilde{\theta}(t), \tilde{\rho}(t), \tilde{\omega}(t))$ by

$$
(\tilde{r}(t), \tilde{\theta}(t), \tilde{\rho}(t), \tilde{\omega}(t))=F_{R_{2}} \circ \exp t H_{p_{\kappa_{0}}} \circ F_{R_{2}}^{-1}(r, \theta, \rho, \omega),
$$

where $F_{R_{2}}(r, \theta, \rho, \omega)=\left(r / R_{2}, \theta, \rho, \omega / R_{2}\right)$. By (4.4), we have for all $(x, \theta, \rho, \nu) \in F_{R_{2}} \Omega^{+}\left(R_{2}, \tilde{U}_{\kappa_{0}}, J, \sigma\right)$, $0 \leq t \leq R_{2} t_{1}$ and $\gamma=(j, \alpha, k, \beta)$,

$$
\left|\partial^{\gamma}(\tilde{r}(t)-x)\right|+\left|\partial^{\gamma}(\tilde{\theta}(t)-\theta)\right|+\left|\partial^{\gamma}(\tilde{\rho}(t)-\rho)\right|+\left|\partial^{\gamma}(\tilde{\omega}(t)-\nu)\right| \leq C_{\gamma} R_{2}^{-j-|\beta|} t_{1},
$$

and hence

$$
\left|\partial^{\gamma}(\partial \tilde{\varphi}(t)-\mathrm{Id})\right| \leq C t_{1}<1 / 2,
$$

where $x=r / R_{2}, \nu=\omega / R_{2}$ and $\partial^{\gamma}=\partial_{r}^{j} \partial_{\theta}^{\alpha} \partial_{\rho}^{k} \partial_{\omega}^{\beta}$. For all $0 \leq t \leq R_{2} t_{1}$ and $(r, \theta, \rho, \omega) \in$ $\tilde{\varphi}\left(t, F_{R_{2}} \Omega^{+}\left(R_{2}, \tilde{U}_{\kappa_{0}}, J, \sigma\right)\right), \tilde{\varphi}(t)$ thus has the inverse

$$
\tilde{\varphi}(t)^{-1}=\left(\tilde{r}(t)^{-1}, \tilde{\theta}(t)^{-1}, \tilde{\rho}(t)^{-1}, \tilde{\omega}(t)^{-1}\right),
$$

and $\tilde{\varphi}(t)^{-1}$ satisfies

$$
\begin{gathered}
\left|\partial^{\gamma}\left(\tilde{r}(t)^{-1}-x\right)\right|+\left|\partial^{\gamma}\left(\tilde{\theta}(t)^{-1}-\theta\right)\right| \leq C_{\gamma} R_{2}^{-j-|\beta|} t_{1}, \\
\left|\partial^{\gamma}\left(\tilde{\rho}(t)^{-1}-\rho\right)\right|+\left|\partial^{\gamma}\left(\tilde{\omega}(t)^{-1}-\nu\right)\right| \leq C_{\gamma} R_{2}^{-j-|\beta|} t_{1} .
\end{gathered}
$$

After the rescaling $(x, \nu) \mapsto\left(R_{2} x, R_{2} \nu\right)$, we see that

$$
\left(r(t)^{-1}, \theta(t)^{-1}, \rho(t)^{-1}, \omega(t)^{-1}\right)=\left(\exp t H_{p_{\kappa_{0}}}\right)^{-1}(r, \theta, \rho, \omega)
$$

exists for all $(r, \theta, \rho, \omega) \in \exp t H_{p_{\kappa_{0}}}\left(\Omega^{+}\left(R_{2}, \tilde{U}_{\kappa_{0}}, J, \sigma\right)\right)$ and $0 \leq t \leq R_{2} t_{1}$, and satisfies

$$
\begin{gathered}
\left|\partial^{\gamma}\left(r(t)^{-1}-r\right)\right|+\left|\partial^{\gamma}\left(\omega(t)^{-1}-\omega\right)\right| \leq C_{j \alpha k \beta} R_{2}^{1-j-|\beta|} t_{1}, \\
\left|\partial^{\gamma}\left(\theta(t)^{-1}-\theta\right)\right|+\left|\partial^{\gamma}\left(\rho(t)^{-1}-\rho\right)\right| \leq C_{j \alpha k \beta} R_{2}^{-j-|\beta|} t_{1} .
\end{gathered}
$$

We now define $b_{j}^{+}(t)$ inductively as follows. Put

$$
b_{0}^{+}(t, r, \theta, \rho, \omega)=b^{+} \circ\left(\exp t H_{p_{\kappa_{0}}}\right)^{-1}(r, \theta, \rho, \omega) \quad \text { on } \quad \exp t H_{p_{\kappa_{0}}}\left(\operatorname{supp} b^{+}\right),
$$


and $b_{0}^{+}(t, r, \theta, \rho, \omega)=0$ outside $\exp t H_{p_{\kappa_{0}}}\left(\operatorname{supp} b^{+}\right)$. By $(6.3),\left(b_{0}^{+}(t)\right)_{0 \leq t \leq R_{2} t_{1}}$ is bounded in $S_{\mathrm{sc}}\left(\exp t H_{p_{\kappa_{0}}}\left(\operatorname{supp} b^{+}\right)\right)$. It is well known that $b_{0}^{+}(t)$ solves the first transport equation

$$
\frac{\partial b_{0}^{+}}{\partial t}+\left\{p_{\kappa_{0}}, b_{0}^{+}\right\}=0, \quad b_{0}^{+}(0)=b^{+},
$$

where $\{\cdot, \cdot\}$ is the Poisson bracket. Moreover, a standard semiclassical symbolic calculus yields that

$$
\frac{\partial}{\partial t} \mathrm{Op}_{\kappa_{0}, h}\left(b_{0}^{+}(t)\right)+\frac{i}{h}\left[h^{2} \widehat{P}_{\kappa_{0}}, \mathrm{Op}_{\kappa_{0}, h}\left(b_{0}^{+}(t)\right)\right]=h \mathrm{Op}_{\kappa_{0}, h}\left(r_{0}^{+}(t)\right),
$$

where $r_{0}^{+}(t)$ is supported in $\operatorname{supp} b_{0}^{+}(t)$ modulo $O\left(h^{\infty}\right)$ on $L^{2}(\widehat{M})$, i.e., for all $n \geq 0$, there exists a symbol $\tilde{r}_{0}^{+}(t) \in S_{\mathrm{sc}}\left(\operatorname{supp} b_{0}^{+}(t)\right)$ such that

$$
\left\|\mathrm{Op}_{\kappa_{0}, h}\left(r_{0}^{+}(t)\right)-\mathrm{Op}_{\kappa_{0}, h}\left(\tilde{r}_{0}^{+}(t)\right)\right\|_{\mathcal{L}\left(L^{2}(\widehat{M})\right)} \leq C_{n} h^{n}, \quad h \in(0,1],
$$

for $0 \leq t \leq R_{2} t_{1}$. Next, put

$$
\left.b_{1}^{+}(t, r, \theta, \rho, \omega)\right)=\int_{0}^{t} \tilde{r}_{0}^{+}\left(s, \exp s H_{p_{\kappa_{0}}} \circ\left(\exp t H_{p_{\kappa_{0}}}\right)^{-1}(r, \theta, \rho, \omega)\right) d s
$$

on $\exp t H_{p_{\kappa_{0}}}\left(\operatorname{supp} b^{+}\right)$, and $\left.b_{1}^{+}(t, r, \theta, \rho, \omega)\right)=0$ otherwise. Again (6.3) implies $\left(b_{1}^{+}(t)\right)_{0 \leq t \leq R_{2} t_{1}}$ is bounded in $S_{\mathrm{sc}}\left(\exp t H_{p_{\kappa_{0}}}\left(\operatorname{supp} b^{+}\right)\right) . b_{1}^{+}(t)$ is a solution to the second transport equation

$$
\frac{\partial b_{1}^{+}}{\partial t}+\left\{p_{\kappa_{0}}, b_{1}^{+}\right\}=\tilde{r}_{0}^{+}, \quad b_{0}^{+}(0)=0,
$$

which implies

$$
\frac{\partial}{\partial t} \mathrm{Op}_{\kappa_{0}, h}\left(b_{0}^{+}(t)+h b_{1}^{+}(t)\right)+\frac{i}{h}\left[h^{2} \widehat{P}_{\kappa_{0}}, \mathrm{Op}_{\kappa_{0}, h}\left(b_{0}^{+}(t)+h b_{1}^{+}(t)\right)\right]=h^{2} \mathrm{Op}_{\kappa_{0}, h}\left(r_{1}^{+}(t)\right),
$$

where $r_{1}^{+}(t)$ is supported in $\operatorname{supp} b_{0}^{+}(t)$ modulo $O\left(h^{\infty}\right)$ on $L^{2}(\widehat{M})$. Iterating this procedure and putting $b_{h}^{+}(t)=\sum_{j=0}^{N} h^{j} b_{j}^{+}(t)$, we have

$$
\frac{\partial}{\partial t} \mathrm{Op}_{\kappa_{0}, h}\left(b_{h}^{+}(t)\right)+\frac{i}{h}\left[h^{2} \widehat{P}_{\kappa_{0}}, \mathrm{Op}_{\kappa_{0}, h}\left(b_{h}^{+}(t)\right)\right]=O\left(h^{N+1}\right),
$$

and $\mathrm{Op}_{\kappa_{0}, h}\left(b_{h}^{+}(0)\right)=\mathrm{Op}_{\kappa_{0}, h}\left(b^{+}\right)$. Integrating the above equation with respect to $t \in\left[0, R_{2} t_{1}\right]$, we obtain the assertion for $t \in\left[0, R_{2} t_{1}\right]$. For general $T>0$, we divide the geodesics into a finite number of small curves, as well as the proof of Corollary 4.3, so that each curve is contained some fixed coordinate neighborhood. Applying the above argument on each chart, we have the assertion by a partition of unity argument.

The following tells us that the support of $e^{-i t h \widehat{P}} \mathrm{Op}_{\kappa_{0}}\left(b_{l}^{+}\right)$is essentially away from the support of $\mathrm{Op}_{\kappa_{0}}\left(a_{l}^{+}\right)$if $t=O(r)$, which is crucial to prove Theorem 6.1.

Proposition 6.3. There exists $c_{0}>0$ that for all $0<\varepsilon<1 / 2$ and $t_{1}>0$, if we choose $\delta_{\varepsilon, t_{1}}<c_{0} \varepsilon^{2}\left\langle 1 / t_{1}\right\rangle^{-1}$ and $L_{\varepsilon, t_{1}}=\delta_{\varepsilon, t_{1}}^{-1}$, then for all $\left(\sigma_{l}\right)_{0 \leq l \leq L_{\varepsilon, t_{1}}} \subset(-1,1 / 2]$ satisfying $(2.12)$ with $\delta=\delta_{\varepsilon, t_{1}}, L=L_{\varepsilon, t_{1}}$ and all $t \geq R_{2} t_{1}$,

$$
\exp t H_{p} \kappa_{0 *}^{-1} \Omega_{i}^{+}\left(R_{2}, \tilde{U}_{\kappa_{0}}, J, \varepsilon, \delta_{\varepsilon, t_{1}}, l\right) \cap \kappa_{0 *}^{-1} \Gamma_{i}^{+}\left(R_{1}, \tilde{U}_{\kappa_{0}}, J, \varepsilon, \delta_{\varepsilon, t_{1}}, l\right)=\emptyset .
$$

Proof. It suffices to show that (6.4) holds with $\Omega_{i}^{+}\left(R_{2}, \tilde{U}_{\kappa_{0}}, J, \varepsilon, \delta_{\varepsilon, t_{1}}, l\right)$ replaced by

$$
\Omega_{i}^{+}\left(R_{1}, R_{2}, \tilde{U}_{\kappa_{0}}, J, \varepsilon, \delta_{\varepsilon, t_{1}}, l\right) \text {, }
$$

since $\Omega_{i}^{+}\left(R_{2}, \tilde{U}_{\kappa_{0}}, J, \varepsilon, \delta_{\varepsilon, t_{1}}, l\right) \subset \Omega_{i}^{+}\left(R_{1}, R_{2}, \tilde{U}_{\kappa_{0}}, J, \varepsilon, \delta_{\varepsilon, t_{1}}, l\right)$. We also note that, by Corollary $4.3, \Omega_{i}^{+}\left(R_{1}, R_{2}, \tilde{U}_{\kappa_{0}}, J, \varepsilon, \delta_{\varepsilon, t_{1}}, l\right)$ is invariant with respect to $\rho$ under the geodesic flow and coordinate transformations. Therefore, by the definition of the intermediate regions and the energy conservation law, it suffices to check that

$$
\frac{\rho(t)}{\sqrt{2 E_{0}}}>\frac{\rho}{\sqrt{2 E_{0}}}+2 \delta_{\varepsilon, t_{1}}, \quad t \geq R_{2} t_{1}
$$


where $E_{0}=p_{\kappa_{0}}(r, \theta, \rho, \omega)$ and $(r, \theta, \rho, \omega)$ belongs to

$$
\left\{R_{1}<r<4 R_{2}, \theta \in \tilde{U}_{\kappa_{0}}, E_{0} \in J, \rho / \sqrt{2 E_{0}} \in\left[-1 / 2, \sqrt{1-\varepsilon^{2} / 4}\right]\right\} .
$$

Note that all $\Omega_{i}^{+}\left(R_{1}, R_{2}, \tilde{U}_{\kappa_{0}}, J, \varepsilon, \delta_{\varepsilon, t_{1}}, l\right)$ are contained in the above region. By (4.1) and (4.3), for sufficiently large $r>R_{1}$ and all $t \geq 0$,

$$
\dot{\rho}(t) \geq \frac{1}{r(t)^{3}}\left(h^{j k}(\theta(t))-O\left(r(t)^{-\mu}\right)\right) \omega_{j}(t) \omega_{k}(t) \gtrsim(r+|t|)^{-3} r^{2} \varepsilon^{2} \sqrt{2 E_{0}} .
$$

Since

$$
\int_{0}^{R_{2} t_{1}}(r+|t|)^{-3} r^{2} d t \gtrsim \frac{R_{2} t_{1}}{r+R_{2} t_{1}} \geq \frac{t_{1}}{4+t_{1}},
$$

we can find $c_{0}>0$ small enough such that

$$
\frac{\rho\left(R_{2} t_{1}\right)}{\sqrt{2 E_{0}}} \geq \frac{\rho}{\sqrt{2 E_{0}}}+2 c_{0} \varepsilon^{2} \frac{t_{1}}{1+t_{1}}>\frac{\rho}{\sqrt{2 E_{0}}}+2 \delta_{\varepsilon, t_{1}},
$$

which implies the assertion since $\dot{\rho}(t)$ is non-negative.

Proof of Theorem 6.1. Since (4.5) implies

$$
\left|\sqrt{2 E_{0}}-\rho(t)\right| \lesssim R_{2} /\left(R_{2}+|t|\right), \quad t \geq 0,
$$

it follows from (4.17) and (4.18) that there exists a constant $C_{0}>0$ such that for any $0<\varepsilon_{0}<$ $1 / 2$, we can find $T_{\varepsilon_{0}}>0$ such that

$$
\begin{aligned}
& \exp R_{2} T_{\varepsilon_{0}} H_{p}\left(\kappa_{0 *}^{-1} \Omega_{i}^{+}\left(R_{2}, \tilde{U}_{\kappa_{0}}, J, \varepsilon, \delta_{\varepsilon, t_{1}}, l\right)\right) \\
& \subset \bigcup_{\kappa} \kappa_{*}^{-1} \Omega_{s}^{+}\left(R_{2} T_{\varepsilon_{0}} / C_{0}, C_{0} R_{2} T_{\varepsilon_{0}}, \tilde{U}_{\kappa}, J, \varepsilon_{0}\right),
\end{aligned}
$$

where $\tilde{U}_{\kappa} \Subset U_{\kappa}$. Note that $C_{0}$ and $T_{\varepsilon_{0}}$ may be taken uniformly with respect to $R_{2}$. We fix such a $T_{\varepsilon_{0}}$ with $T_{\varepsilon_{0}} \geq C_{0}$. By Theorem 6.2, we obtain that for all $N \geq 0$,

$$
\mathrm{Op}_{\kappa_{0}}\left(a_{l}^{+}\right) e^{-i t h \widehat{P}} \mathrm{Op}_{\kappa_{0}}\left(b_{l}^{+}\right)=\mathrm{Op}_{\kappa_{0}}\left(a_{l}^{+}\right) \sum_{\kappa} \mathrm{Op}_{\kappa}\left(b_{\kappa, h}^{+}(t)\right) e^{-i t h \widehat{P}}+O\left(h^{N+1}|t|\right)
$$

on $L^{2}(\widehat{M})$ uniformly with respect to $R_{2}$ and $0 \leq t \leq R_{2} T_{\varepsilon_{0}}$, where

$$
b_{\kappa, h}^{+}(t) \in S_{\mathrm{sc}}\left(\kappa_{*} \exp t H_{p}\left(\kappa_{0 *}^{-1} \Omega_{i}^{+}\left(R_{2}, \tilde{U}_{\kappa_{0}}, J, \varepsilon, \delta_{\varepsilon, t_{1}}, l\right)\right)\right) .
$$

Suppose that $R_{2} t_{1} \leq t \leq R_{2} T_{\varepsilon_{0}}$. Since the support of $a_{l}^{+}$does not intersect with the support of $\left(\kappa \circ \kappa_{0}^{-1}\right)_{*} b_{\kappa, h}^{+}(t)$ for any $\kappa$ with $V_{\kappa_{0}} \cap V_{\kappa} \neq \emptyset$ by Proposition 6.3 , The semiclassical symbolic calculus (see subsection 2.2) implies that the above operator is bounded on $L^{2}(\widehat{M})$ with the norm dominated by $C_{N} h^{N+1}\langle t\rangle$, where the constant $C_{N}>0$ may be taken uniformly with respect to $R_{2}, t$ and $h$. If $R_{2} T_{\varepsilon_{0}} \geq h^{-1}$, then we obtain (6.1). We thus assume $R_{2} T_{\varepsilon_{0}} \leq t \leq h^{-1}$. By (6.5) and Theorem 6.2 , there exist symbols $b_{\kappa, h}^{+}\left(R_{2} T_{\varepsilon_{0}}\right) \in S_{\mathrm{sc}}\left(\Omega_{s}^{+}\left(R_{2} T_{\varepsilon_{0}} / C_{0}, C_{0} R_{2} T_{\varepsilon_{0}}, \tilde{U}_{\kappa}, J, \varepsilon_{0}\right)\right)$ such that

$$
\begin{aligned}
e^{-i t h \widehat{P}} \mathrm{Op}_{\kappa_{0}}\left(b_{l}^{+}\right) & =e^{-i\left(t-R_{2} T_{\varepsilon_{0}}\right) h \widehat{P}} e^{-i R_{2} T_{\varepsilon_{0}} h \widehat{P}} \mathrm{Op}_{\kappa_{0}}\left(b_{l}^{+}\right) \\
& =e^{-i\left(t-R_{2} T_{\varepsilon_{0}}\right) h \widehat{P}} \sum_{\kappa} \mathrm{Op}_{\kappa}\left(b_{\kappa, h}^{+}\left(R_{2} T_{\varepsilon_{0}}\right)\right) e^{-i R_{2} T_{\varepsilon_{0}} h \widehat{P}}+O\left(h^{N}\right)
\end{aligned}
$$

on $L^{2}(\widehat{M})$. Put $B=\sum_{\kappa} \operatorname{Op}_{\kappa}\left(b_{\kappa, h}^{+}\left(R_{2} T_{\varepsilon_{0}}\right)\right)$ and divide $B$ as follows

$$
B=\varphi_{\kappa_{0}} B \tilde{\varphi}_{\kappa_{0}}+\left(1-\varphi_{\kappa_{0}}\right) B \tilde{\varphi}_{\kappa_{0}}+B\left(1-\tilde{\varphi}_{\kappa_{0}}\right),
$$

where $\varphi_{\kappa_{0}}, \tilde{\varphi}_{\kappa_{0}} \in C^{\infty}\left(\widetilde{M}_{\infty}\right)$ such that

$$
\operatorname{supp} \tilde{\varphi}_{\kappa_{0}} \subset \operatorname{supp} \varphi_{\kappa_{0}} \subset(2, \infty) \times V_{\kappa_{0}}, \quad \varphi_{\kappa_{0}} \equiv 1 \text { close to } \operatorname{supp} \tilde{\varphi}_{\kappa_{0}} .
$$

Since $\operatorname{supp}\left(1-\varphi_{\kappa_{0}}\right) \cap \operatorname{supp} \tilde{\varphi}_{\kappa_{0}}=\emptyset$, the second term is $O\left(h^{\infty}\right)$ on $L^{2}(\widehat{M})$. The third term is also $O\left(h^{\infty}\right)$ on $L^{2}(\widehat{M})$ since $\operatorname{supp} b_{\kappa, h}^{+}\left(R_{2} T_{\varepsilon_{0}}\right) \cap \operatorname{supp}\left(1-\tilde{\varphi}_{\kappa_{0}}\right)=\emptyset$, which follows from the facts

$$
r>R_{2}>2 \quad \text { on } \operatorname{supp} b_{\kappa, h}^{+}\left(R_{2} T_{\varepsilon_{0}}\right), \quad 0<r<2 \quad \text { on } \operatorname{supp}\left(1-\tilde{\varphi}_{\kappa_{0}}\right) .
$$


By invariance properties of the strongly outgoing region and $h$-PDO under coordinates transformations, we can write

$$
\varphi_{\kappa_{0}} B \tilde{\varphi}_{\kappa_{0}}=\mathrm{Op}_{\kappa_{0}}\left(\tilde{b}_{\kappa_{0}, h}^{+}\left(R_{2} T_{\varepsilon_{0}}\right)\right)+O\left(h^{\infty}\right)
$$

on $L^{2}(\widehat{M})$ with some $\tilde{b}_{\kappa_{0}, h}^{+}\left(R_{2} T_{\varepsilon_{0}}\right) \in S_{\mathrm{sc}}\left(\Omega_{s}^{+}\left(R_{2} T_{\varepsilon_{0}} / C_{0}, C_{0} R_{2} T_{\varepsilon_{0}}, \tilde{U}_{\kappa_{0}}^{\prime}, J, \varepsilon_{0}\right)\right)$, where $\tilde{U}_{\kappa_{0}}^{\prime} \Subset U_{\kappa_{0}}$. Consider a splitting of the interval $\left[R_{2} T_{\varepsilon_{0}} / C_{0}, C_{0} R_{2} T_{\varepsilon_{0}}\right]$ :

$$
R_{2} T_{\varepsilon_{0}} / C_{0}=\tilde{R}_{0}<\tilde{R}_{1}<\tilde{R}_{2}<\cdots<\tilde{R}_{k}, \tilde{R}_{j}=2^{j} \tilde{R}_{0}, \tilde{R}_{k-1}<C_{0} R_{2} T_{\varepsilon_{0}}<\tilde{R}_{k} .
$$

Clearly $2^{k} \leq 2 C_{0}^{2}$. Using a method of the partition of unity, we split $\tilde{b}_{\kappa_{0}, h}^{+}\left(R_{2} T_{\varepsilon_{0}}\right)$ as $\tilde{b}_{\kappa_{0}, h}^{+}\left(R_{2} T_{\varepsilon_{0}}\right)=$ $\sum_{j=0}^{k-2} \tilde{b}_{\kappa_{0}, h, j}^{+}$with $\tilde{b}_{\kappa_{0}, h, j}^{+} \in S_{\mathrm{sc}}\left(\Omega_{s}^{+}\left(\tilde{R}_{j}, \tilde{U}_{\kappa_{0}}^{\prime}, J, \varepsilon_{0}\right)\right)$. By Theorem 5.10, we can construct the Isozaki-Kitada parametrix of $e^{-i\left(t-R_{2} T_{\varepsilon_{0}}\right) h \widehat{P}} \mathrm{Op}_{\kappa_{0}}\left(\tilde{b}_{\kappa_{0}, h, j}^{+}\right)$for sufficiently large $R_{2} \geq R_{\mathrm{IK}}, \lambda \geq \lambda_{\mathrm{IK}}$ and small $0<\varepsilon_{0} \leq \varepsilon_{\mathrm{IK}}$, and we obtain

$$
\begin{aligned}
& e^{-i t h \widehat{P}} \mathrm{Op}_{\kappa_{0}}\left(b_{l}^{+}\right) \\
& =\sum_{j=0}^{k-2} \kappa_{0}^{*} I_{\mathrm{IK}}^{+}\left(c_{h, j}^{+}\right) e^{-i\left(t-R_{2} T_{\varepsilon_{0}}\right) h \frac{1}{2} D_{r}^{2}} I_{\mathrm{IK}}^{+}\left(d_{h, j}^{+}\right)^{*} \kappa_{0 *} e^{-i R_{2} T_{\varepsilon_{0}} h \widehat{P}}+Q\left(t, h, N, R_{2}\right),
\end{aligned}
$$

where, for each $j, I_{\mathrm{IK}}^{+}\left(c_{h, j}^{+}\right)$and $I_{\mathrm{IK}}^{+}\left(d_{h, j}^{+}\right)$are FIO's defined in Definition 5.6 with some phase function $S_{\kappa_{0}, j}^{+} \in C^{\infty}\left(\mathbb{R}^{d} ; \mathbb{R}\right)$, which satisfies the statement of Theorem 5.1 with $R=\tilde{R}_{j}$, and some amplitudes

$$
\begin{aligned}
& c_{h, j}^{+} \in S_{\mathrm{sc}}\left(\Omega_{s}^{+}\left(\lambda^{-3} \tilde{R}_{j}, \lambda^{3} \tilde{R}_{j}, \tilde{U}_{\kappa_{0}}^{\prime}, J, \lambda^{3} \varepsilon_{0}\right)\right), \\
& d_{h, j}^{+} \in S_{\mathrm{sc}}\left(\Omega_{s}^{+}\left(\lambda^{-1} \tilde{R}_{j}, \lambda \tilde{R}_{j}, \tilde{U}_{\kappa_{0}}^{\prime}, J, \lambda \varepsilon_{0}\right)\right) .
\end{aligned}
$$

The remainder term $Q\left(t, h, N, R_{2}\right)$ is uniformly bounded on $L^{2}(\widehat{M})$ with the norm of order $h^{N}$ with respect to $R_{2}$ and $R_{2} T_{\varepsilon_{0}} \leq t \leq h^{-1}$. By the composition rule of FIO's with PDO's, $a_{l}^{+}\left(r, \theta, h D_{r}, h D_{\theta}\right) I_{\mathrm{IK}}^{+}\left(c_{h, j}^{+}\right)$is also FIO's (up to the smoothing term $O\left(h^{\infty}\right)$ on $L^{2}(\widehat{M})$ ) with the phase $S_{\kappa_{0}, j}^{+}$and the amplitude supported in

$$
X=\left\{(r, \theta, \rho, \omega) \mid a_{l}^{+}\left(r, \theta, \partial_{r} S_{\kappa_{0}, j}^{+}, \partial_{\theta} S_{\kappa_{0}, j}^{+}\right) c_{h, j}^{+}(r, \theta, \rho, \omega) \neq 0\right\} .
$$

By the support property of $a_{l}^{+}$, we see that

$$
\partial_{r} S_{\kappa_{0}, j}^{+} \leq \sqrt{2\left(1-\varepsilon^{2} / 4\right) p_{\kappa_{0}}\left(r, \theta, \partial_{r} S_{\kappa_{0}, j}^{+}, \partial_{\theta} S_{\kappa_{0}, j}^{+}\right)} .
$$

Since $(r, \theta, \rho, \omega) \in \operatorname{supp} c_{h, j}^{+} \subset \Omega_{s}^{+}\left(\lambda^{-3} \tilde{R}_{j}, \lambda^{3} \tilde{R}_{j}, \tilde{U}_{\kappa_{0}}, J, \lambda^{3} \varepsilon_{0}\right)$, by the above estimate and (5.1), we have

$$
\rho / \sqrt{2 p_{\kappa_{0}}(r, \theta, \rho, \omega)} \leq \sqrt{1-\varepsilon^{2} / 4}-C\left|\lambda^{3} \varepsilon_{0}\right|^{2} \leq \sqrt{1-\varepsilon^{2} / 8} .
$$

On the other hand, choosing $\varepsilon_{0}>0$ small enough so that $\lambda^{3} \varepsilon_{0}<\varepsilon^{2} / 8$ (note that $\lambda>\lambda_{\text {IK }}$ is fixed), the support property of $c_{h, j}^{+}$implies

$$
\rho / \sqrt{2 p_{\kappa_{0}}(r, \theta, \rho, \omega)} \geq \sqrt{1-\lambda^{3} \varepsilon_{0}}>\sqrt{1-\varepsilon^{2} / 8} .
$$

The above two inequalities show that $X=\emptyset$ and hence $a_{l}^{+}\left(r, \theta, h D_{r}, h D_{\theta}\right) I_{\mathrm{IK}}^{+}\left(c_{h, j}^{+}\right)$is $O\left(h^{\infty}\right)$ in $L^{2}(\widehat{M})$. Since $\kappa_{0}^{*} e^{-i\left(t-R_{2} T_{\varepsilon_{0}}\right) h \frac{1}{2} D_{r}^{2}} I_{\mathrm{IK}}^{+}\left(d_{h, j}^{+}\right)^{*} \kappa_{0 *} e^{-i R_{2} T_{\varepsilon_{0}} h \widehat{P}}$ is uniformly bounded on $L^{2}(\widehat{M})$ with respect to $R_{2}, h$ and $t$, we obtain the assertion and conclude the proof.

\section{The WKB PARAMETRIX}

In the previous section we proved that $\mathrm{Op}_{\kappa_{0}, h}\left(a_{l}^{ \pm}\right) e^{-i t h \widehat{P}} \mathrm{Op}_{\kappa_{0}, h}\left(b_{l}^{ \pm}\right)$are rapidly decaying with respect to $h \in(0,1]$ if $R t_{1} \leq \pm t \leq h^{-1}$ with any $t_{1}>0$ and large $R>0$. Therefore, it remains to control the above operators for $0 \leq \pm t \leq R t_{0}$ with sufficiently small $t_{0}$. This section discuss construction of the WKB parametrix of propagator $e^{-i t \widehat{P}} \mathrm{Op}_{\kappa}\left(a^{ \pm}\right)$for $0 \leq \pm t \leq R t_{0}$, where $a^{+}$ (resp. $a^{-}$) is supported in an outgoing (resp. incoming) region. By (4.18), we can always work on a fixed coordinate neighborhood $U_{\kappa}$ and hence do not write the subscript $\kappa$ explicitly. Let 
$\tilde{U}_{\kappa} \Subset U_{\kappa}$ be as in Example 2.2 and fix open subsets $U \Subset U_{0} \Subset U_{1} \Subset U_{2} \Subset \tilde{U}_{\kappa}$, open intervals $J \Subset J_{0} \Subset J_{1} \Subset J_{2} \Subset(0, \infty)$ and constants $-1<\sigma<\sigma_{0}<\sigma_{1}<\sigma_{2}<1$ arbitrarily.

7.1. Fourier integral operators for the WKB parametrix. We here study time dependent FIO's which will be used to construct the WKB parametrix. We first construct the phase function.

Theorem 7.1. We can choose $t_{0}>0$ small enough such that, for sufficiently large $R_{2}>0$ and all $R_{1}>R_{2}$, there exist smooth and real-valued functions

$$
\Psi^{+} \in C^{\infty}\left(\left(0, R_{1} t_{0}\right) \times \mathbb{R}^{2 d}\right), \quad \Psi^{-} \in C^{\infty}\left(\left(-R_{1} t_{0}, 0\right) \times \mathbb{R}^{2 d}\right),
$$

satisfying the following Hamilton Jacobi equation on $\Gamma^{ \pm}\left(R_{1}, U_{1}, J_{1}, \sigma_{1}\right)$ :

$$
\left\{\begin{array}{l}
\partial_{t} \Psi^{ \pm}+p\left(r, \theta, \partial_{r} \Psi^{ \pm}, \partial_{\theta} \Psi^{ \pm}\right)=0, \quad 0 \leq \pm t \leq R_{1} t_{0}, \\
\left.\Psi^{ \pm}\right|_{t=0}=r \rho+\theta \cdot \omega
\end{array}\right.
$$

such that we have the followings all $0 \leq \pm t \leq R_{1} t_{0}$ :

$$
\begin{gathered}
\operatorname{supp}\left(\Psi^{ \pm}(t, r, \theta, \rho, \omega)-r \rho-\theta \cdot \omega\right) \subset \Gamma^{ \pm}\left(R_{2}, U_{2}, J_{2}, \sigma_{2}\right), \\
\left|\partial_{r}^{j} \partial_{\theta}^{\alpha} \partial_{\rho}^{k} \partial_{\omega}^{\beta}\left(\Psi^{ \pm}(t, r, \theta, \rho, \omega)-r \rho-\theta \cdot \omega\right)\right| \leq C_{j \alpha k \beta}\langle r\rangle^{-j-|\beta|}|t| \quad \text { on } \quad \mathbb{R}^{2 d} .
\end{gathered}
$$

Moreover, for all $(r, \theta, \rho, \omega) \in \Gamma^{ \pm}\left(R_{1}, U_{1}, J_{1}, \sigma_{1}\right)$, we have

$$
\left|\partial_{r}^{j} \partial_{\theta}^{\alpha} \partial_{\rho}^{k} \partial_{\omega}^{\beta}\left(\Psi^{ \pm}(t, r, \theta, \rho, \omega)-r \rho-\theta \cdot \omega+t p(r, \theta, \rho, \omega)\right)\right| \leq C_{j \alpha k \beta} r^{-j-|\beta|}|t| t_{0} .
$$

Proof. This theorem can be proved similarly to Theorem 5.1. We only prove the theorem for the case $t \geq 0$, and the proof for the case $t \leq 0$ is similar. Let $R_{1}>R_{1}^{\prime}>R_{1} / 2, U_{1} \Subset U_{1}^{\prime} \Subset \tilde{U}_{\kappa}$, $J_{1} \Subset J_{1}^{\prime} \Subset(0, \infty)$ and $\sigma_{1}<\sigma_{1}^{\prime}<1$. Let $F_{R_{1}}:(r, \theta, \rho, \omega) \mapsto\left(r / R_{1}, \theta, \rho, \omega\right)$, and define $g^{+}(t)$ and $\tilde{g}^{+}(t)$ by

$$
\begin{aligned}
g^{+}(t) & :=(r(t, r, \theta, \rho, \omega), \theta(t, r, \theta, \rho, \omega), \rho, \omega), \\
\tilde{g}^{+}(t) & =(\tilde{r}(t, x, \theta, \rho, \omega), \tilde{\theta}(t, x, \theta, \rho, \omega), \rho, \omega) \\
& :=F_{R_{1}} \circ g^{+}(t) \circ F_{R_{1}}^{-1}(x, \theta, \rho, \omega),
\end{aligned}
$$

where $(r(t, r, \theta, \rho, \omega), \theta(t, r, \theta, \rho, \omega))$ is the Hamilton flow generated by $p$, and $x=r / R_{1}$. By Proposition 4.2, we have

$$
\begin{aligned}
& \left|\partial_{x}^{j} \partial_{\theta}^{\alpha} \partial_{\rho}^{k} \partial_{\omega}^{\beta}(\tilde{r}(t)-x)\right| \leq C_{j \alpha k \beta} R_{1}^{-1}|t| \leq C_{j \alpha k \beta} t_{0}, \\
& \left|\partial_{x}^{j} \partial_{\theta}^{\alpha} \partial_{\rho}^{k} \partial_{\omega}^{\beta}(\tilde{\theta}(t)-\theta)\right| \leq C_{j \alpha k \beta} R_{1}^{\prime-1}|t| \leq C_{j \alpha k \beta} t_{0},
\end{aligned}
$$

and

$$
\left|\partial \tilde{g}^{+}(t)-\operatorname{Id}\right| \leq C t_{0}<1 / 2,
$$

for all $(x, \theta, \rho, \omega) \in F_{R_{1}} \Gamma^{+}\left(R_{1}^{\prime}, U_{1}^{\prime}, J_{1}^{\prime}, \sigma_{1}^{\prime}\right)$ and $0 \leq t \leq R_{1} t_{0}$ as long as $t_{0}>0$ is small enough. Applying a same argument as that in the proof of Lemma 5.3 to $\tilde{g}^{+}(t)$, we see that $g^{+}(t)$ is diffeomorphic from $\Gamma^{+}\left(R_{1}^{\prime}, U_{1}^{\prime}, J_{1}^{\prime}, \sigma_{1}^{\prime}\right)$ onto its range for all $0 \leq t \leq R_{1} t_{0}$, and satisfies

$$
\Gamma^{+}\left(R_{1}, U_{1}, J_{1}, \sigma_{1}\right) \subset g^{ \pm}(t)\left(\Gamma^{+}\left(R_{1}^{\prime}, U_{1}^{\prime}, J_{1}^{\prime}, \sigma_{1}^{\prime}\right)\right), \quad 0 \leq \pm t \leq R_{1} t_{0} .
$$

Let $\Gamma^{+}\left(R_{1}, U_{1}, J_{1}, \sigma_{1}\right) \ni(r, \theta, \rho, \omega) \mapsto\left(\hat{r}^{+}(t), \hat{\theta}^{+}(t), \rho, \omega\right)$ be the inverse of $g^{+}(t)$, and put $\left(r^{+}(t, s), \theta^{+}(t, s), \rho^{+}(t, s), \omega^{+}(t, s)\right):=(r, \theta, \rho, \omega)\left(s, \hat{r}^{+}(t), \hat{\theta}^{+}(t), \rho, \omega\right)$ for $0 \leq s \leq t \leq R_{1} t_{0}$. Then, by a same argument as that in the proof of Lemma 5.4 and Lemma 5.5, we have

$$
\left\{\begin{array}{c}
\left|\partial_{r}^{j} \partial_{\theta}^{\alpha} \partial_{\rho}^{k} \partial_{\omega}^{\beta}\left(\hat{r}^{+}(t)-r\right)\right|+\left|\partial_{r}^{j} \partial_{\theta}^{\alpha} \partial_{\rho}^{k} \partial_{\omega}^{\beta}\left(\omega^{+}(t, t)-\omega\right)\right| \leq C_{j \alpha k \beta} r^{-j-|\beta|}|t|, \\
\left|\partial_{r}^{j} \partial_{\theta}^{\alpha} \partial_{\rho}^{k} \partial_{\omega}^{\beta}\left(\hat{\theta}^{+}(t)-\theta\right)\right|+\left|\partial_{r}^{j} \partial_{\theta}^{\alpha} \partial_{\rho}^{k} \partial_{\omega}^{\beta}\left(\rho^{+}(t, t)-\rho\right)\right| \leq C_{j \alpha k \beta} r^{-j-|\beta|} t_{0},
\end{array}\right.
$$

Define $\tilde{\Psi}^{+} \in C^{\infty}\left(\left(0, R_{1} t_{0}\right) \times \Gamma^{+}\left(R_{1}, U_{1}, J_{1}, \sigma_{1}\right)\right)$ by

$$
\tilde{\Psi}^{+}(t):=r \rho+\theta \cdot \omega+\int_{0}^{t} L\left(r^{+}(t, s), \theta^{+}(t, s), \rho^{+}(t, s), \omega^{+}(t, s)\right) d s,
$$


where $L=\left(\rho \partial_{\rho} p+\omega \cdot \partial_{\omega} p-p\right)(r, \theta, \rho, \omega)$. By a standard Hamilton-Jacobi theory, it is easy to see that $\tilde{\Psi}^{+}(t)$ solves $(7.1)$ and satisfies

$$
\partial \tilde{\Psi}(t)=\left(\rho^{+}(t, t), \omega^{+}(t, t), \hat{r}^{+}(t), \hat{\theta}^{+}(t)\right) .
$$

By (7.4) and the energy conservation law

$$
p\left(r, \theta, \partial_{r} \tilde{\Psi}^{+}(t), \partial_{\theta} \tilde{\Psi}^{+}(t)\right)=p\left(\hat{r}^{+}(t), \hat{\theta}^{+}(t), \rho, \omega\right),
$$

we have

$$
\left|\partial_{r}^{j} \partial_{\theta}^{\alpha} \partial_{\rho}^{k} \partial_{\omega}^{\beta}\left(p\left(r, \theta, \partial_{r} \tilde{\Psi}^{+}(t), \partial_{\theta} \tilde{\Psi}^{+}(t)\right)-p(r, \theta, \rho, \omega)\right)\right| \leq C_{j \alpha k \beta} r^{-j-|\beta|} t_{0} .
$$

Therefore,

$$
\left|\partial_{r}^{j} \partial_{\theta}^{\alpha} \partial_{\rho}^{k} \partial_{\omega}^{\beta}\left(\tilde{\Psi}^{+}(t)-r \rho-\theta \cdot \omega+t p(r, \theta, \rho, \omega)\right)\right| \leq C_{j \alpha k \beta} r^{-j-|\beta|}|t| t_{0} .
$$

Choose $\chi_{+} \in C^{\infty}\left(\mathbb{R}^{2 d}\right)$ so that

$$
0 \leq \chi_{+} \leq 1, \quad \chi_{+} \equiv 1 \quad \text { on } \quad \Gamma^{+}\left(R_{1}, U_{1}, J_{1}, \sigma_{1}\right), \quad \operatorname{supp} \chi_{+} \subset \Gamma^{+}\left(R_{2}, U_{2}, J_{2}, \sigma_{2}\right),
$$

and that

$$
\left|\partial_{r}^{j} \partial_{\theta}^{\alpha} \partial_{\rho}^{k} \partial_{\omega}^{\beta} \chi_{+}(r, \theta, \rho, \omega)\right| \leq C_{j \alpha k \beta}\langle r\rangle^{-j-|\beta|} \quad \text { on } \quad \mathbb{R}^{2 d} .
$$

We now define $\Psi^{+}(t):=r \rho+\theta \cdot \omega+\chi_{+}\left(\tilde{\Psi}^{+}(t)-r \rho+\theta \cdot \omega\right)$. Clearly, $\Psi(t)^{+}$satisfies the statement of theorem 7.1.

Suppose $\left(a^{ \pm}(t)\right)_{0 \leq \pm t \leq R_{1} t_{0}}$ are bounded in $S_{\mathrm{sc}}\left(\Gamma^{ \pm}\left(R_{1}, U_{1}, J_{1}, \sigma_{1}\right)\right)$, respectively. We define the FIO's for the WKB parametrix $I_{\mathrm{WKB}}^{ \pm}\left(a^{ \pm}(t)\right): \mathcal{S}\left(\mathbb{R}^{d}\right) \rightarrow \mathcal{S}\left(\mathbb{R}^{d}\right)$ by

$$
\begin{aligned}
& I_{\mathrm{WKB}}^{ \pm}\left(a^{ \pm}(t)\right) u(r, \theta) \\
& :=\frac{1}{(2 \pi h)^{d}} \int e^{\frac{i}{h}\left(\Psi^{ \pm}(t, r, \theta, \rho, \omega)-r^{\prime} \rho-\theta^{\prime} \cdot \omega\right)} a^{ \pm}(t, r, \theta, \rho, \omega) u\left(r^{\prime}, \theta^{\prime}\right) d r^{\prime} d \theta^{\prime} d \rho d \omega .
\end{aligned}
$$

Proposition 7.2. $I_{\mathrm{WKB}}^{ \pm}\left(a^{ \pm}(t)\right)$ are bounded on $L^{2}\left(\mathbb{R}^{d}\right)$ uniformly with respect to $0 \leq \pm t \leq R_{1} t_{0}$ :

$$
\left\|I_{\mathrm{WKB}}^{ \pm}\left(a^{ \pm}(t)\right) u\right\|_{L^{2}\left(\mathbb{R}^{d}\right)} \leq C\|u\|_{L^{2}\left(\mathbb{R}^{d}\right)} .
$$

Proof. For $\left(r, \theta, \rho, \omega, r^{\prime}, \theta^{\prime}\right) \in \mathbb{R}^{3 d}$ with $r, r^{\prime}>R_{1}$ and $0 \leq t \leq R_{1} t_{0}$, define the map $\left(\rho_{+}^{1}, \omega_{+}^{1}\right)$ by

$$
\left(\rho_{+}^{1}, \omega_{+}^{1}\right)\left(t, r, \theta, \rho, \omega, r^{\prime}, \theta^{\prime}\right)=\int_{0}^{1}\left(\partial_{r, \theta} \Psi^{+}\right)\left(t, r^{\prime}+s\left(r-r^{\prime}\right), \theta^{\prime}+s\left(\theta-\theta^{\prime}\right), \rho, \omega\right) d s .
$$

By (7.2), we have

$$
\sup _{0 \leq t \leq R_{1} t_{0}}\left(\left|\partial^{\gamma}\left(\rho_{+}^{1}-\rho\right)\right|+R_{1}^{-1}\left|\partial^{\gamma}\left(\omega_{+}^{1}-\omega\right)\right|\right) \leq C_{\gamma} R_{1}^{-j-j^{\prime}-|\beta|} t_{0}
$$

where $\partial^{\gamma}=\partial_{r}^{j} \partial_{\theta}^{\alpha} \partial_{\rho}^{k} \partial_{\omega}^{\beta} \partial_{r^{\prime}}^{j^{\prime}} \partial_{\theta^{\prime}}^{\alpha^{\prime}}$ with $\gamma=\left(j, \alpha, k, \beta, j^{\prime}, \alpha^{\prime}\right)$. By this estimate and a similar argument as in the proof of Lemma 5.9, we obtain that, for all $\left(r, \theta, r^{\prime}, \theta^{\prime}\right) \in \mathbb{R}^{2 d}$ with $r, r^{\prime}>R_{1}$ and $0 \leq t \leq R_{1} t_{0}$, the map $(\rho, \omega) \mapsto\left(\rho_{+}^{1}, \omega_{+}^{1}\right)\left(t, r, \theta, \rho, \omega, r^{\prime}, \theta^{\prime}\right)$ is a diffeomorphism from $\mathbb{R}^{d}$ onto itself provided that $t_{0}>0$ is small enough. We also see that the corresponding inverse $(\rho, \omega) \mapsto$ $\left(\rho_{+}^{2}, \omega_{+}^{2}\right)\left(t, r, \theta, \rho, \omega, r^{\prime}, \theta^{\prime}\right)$ satisfies

$$
\sup _{0 \leq t \leq R_{1} t_{0}}\left(\left|\partial^{\gamma}\left(\rho_{+}^{2}-\rho\right)\right|+R_{1}^{-1}\left|\partial^{\gamma}\left(\omega_{+}^{2}-\omega\right)\right|\right) \leq C_{\gamma} R_{1}^{-j-j^{\prime}-|\beta|} t_{0} .
$$

Using the change of variable $(\rho, \omega) \mapsto\left(\rho_{+}^{2}, \omega_{+}^{2}\right), I_{\mathrm{WKB}}^{+}\left(a^{+}(t)\right) I_{\mathrm{WKB}}^{+}\left(a^{+}(t)\right)^{*}$ can be regarded as a $h$-PDO with the amplitude

$$
a^{+}\left(t, r, \theta, \rho_{+}^{2}, \omega_{+}^{2}\right) \overline{a^{+}\left(t, r^{\prime}, \theta^{\prime}, \rho_{+}^{2}, \omega_{+}^{2}\right)}\left|\operatorname{det} \partial_{\rho, \omega}\left(\rho_{+}^{2}, \omega_{+}^{2}\right)\right| .
$$

By (7.5), this amplitude and its all derivatives with respect to $\partial^{\gamma}$ are bounded on $\mathbb{R}^{3 d}$ uniformly with respect to $0 \leq t \leq R_{1} t_{0}$. The assertion then follows from the Calderón-Vaillancourt theorem and the $L^{2}$-functional calculus. When $-R_{1} t_{0} \leq t \leq 0$, the proof is similar. 
7.2. Construction of the parametrix. The main result in this section is the following.

Theorem 7.3. There exists $R_{\mathrm{WKB}}>0$ large enough and $t_{\mathrm{WKB}}>0$ small enough such that, for all $R>R_{0}>R_{1}>R_{\mathrm{WKB}}, a^{ \pm} \in S_{\mathrm{sc}}\left(\Gamma^{ \pm}(R, U, J, \sigma)\right)$ and $N \geq 0$, we can find

$$
b_{h}^{ \pm}(t)=\sum_{j=0}^{N} h^{j} b_{j}^{ \pm}(t)
$$

with $\left(b_{j}^{ \pm}(t)\right)_{0 \leq \pm t \leq R_{1} t_{\mathrm{WKB}}}$ bounded in $S_{\mathrm{sc}}\left(\Gamma^{ \pm}\left(R_{0}, U_{0}, J_{0}, \sigma_{0}\right)\right)$ such that, for all $h \in(0,1]$ and $0 \leq \pm t \leq R_{1} t_{\mathrm{WKB}}$

$$
\| e^{-i t h \widehat{P}} \operatorname{Op}_{\kappa}\left(a^{ \pm}\right)-\kappa^{*} I_{\mathrm{WKB}}^{ \pm}\left(b_{h}^{ \pm}(t)\right) \kappa_{*}||_{\mathcal{L}\left(L^{2}(\widehat{M})\right)} \leq C_{N} h^{N+1}|t|
$$

where $C_{N}>0$ can be taken uniformly with respect to $h, t$ and $R_{1}$.

Remark 7.4. The essential point of Theorem 7.3 is to construct the parametrix on the time interval $0 \leq|t| \leq R_{1} t_{\mathrm{WKB}}$ which allow us to choose the constant $\delta>0$ in Theorem 3.3 independently with respect to $R_{1}$. When $|t|>0$ is small and independent of $R_{1}$, such a parametrix construction is basically well known (see [18] for the case of elliptic operators on the Euclidean space, and [3] for the case of the Laplace-Beltrami operator on an asymptotically hyperbolic manifold).

We prove the theorem for the case when $t \geq 0$. Put $B_{+}(t)=I_{\mathrm{WKB}}^{+}\left(b_{h}^{+}(t)\right)$. By the Duhamel formula, we have

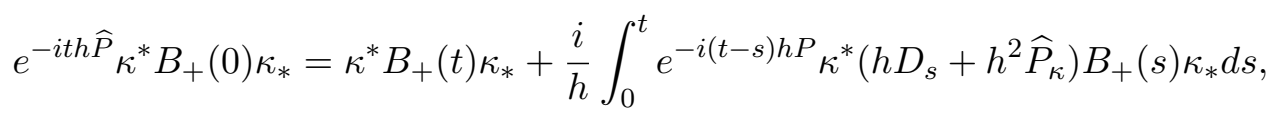

where $D_{s}=i^{-1} \partial_{s}$. Since the off-diagonal decay of the $h$-PDO implies

$$
\left\|\mathrm{Op}_{\kappa}\left(a^{+}\right)-\kappa^{*} a^{+}\left(r, \theta, h D_{r}, h D_{\theta}\right) \kappa_{*}\right\|_{\mathcal{L}\left(L^{2}(\widehat{M})\right)} \leq C_{n} h^{n},
$$

for all $n \geq 0$, it suffices to show that there exists $b_{h}^{+}(t)$ such that $b_{h}^{+}(0)=a^{+}$and

$$
\left\|\left(h D_{s}+h^{2} \widehat{P}_{\kappa}\right) B_{+}(s)\right\|_{\mathcal{L}\left(L^{2}\left(\mathbb{R}^{d}\right)\right)} \leq C_{N} h^{N+2}
$$

for $h \in(0,1]$ and $0 \leq s \leq R_{1} t_{\mathrm{WKB}}$.

Define smooth tensors $x^{+}(t)$ and $y^{+}(t)$ by

$$
x^{+}(t):=\partial_{\rho, \omega} p_{\kappa}\left(r, \theta, \partial_{r} \Psi^{+}(t), \partial_{\theta} \Psi^{+}(t)\right), \quad y^{+}(t):=\left(p+p_{1}\right)\left(r, \theta, \partial_{r}, \partial_{\theta}\right) \Psi^{+}(t) .
$$

Then, $b_{h}^{+}(t)$ satisfying (7.6) can be constructed by solving the following transport equations.

Lemma 7.5. For sufficiently small $t_{0}>0$, there exist

$$
\left(b_{j}^{+}(t)\right)_{0 \leq t \leq R_{1} t_{0}} \text { bounded in } S_{\mathrm{sc}}\left(\Gamma^{ \pm}\left(R_{0}, U_{0}, J_{0}, \sigma_{0}\right)\right), j=0,1, \ldots, N,
$$

such that $b_{j}^{+}(t)$ solve the transport equations:

$$
\left\{\begin{aligned}
\partial_{t} b_{0}^{+}(t)+x^{+}(t) \cdot \partial_{r, \theta} b_{0}^{+}(t)+y^{+}(t) b_{0}^{+}(t) & =0, \\
\partial_{t} b_{j}^{+}(t)+x^{+}(t) \cdot \partial_{r, \theta} b_{j}^{+}(t)+y^{+}(t) b_{j}^{+}(t)+i \widehat{P}_{\kappa} b_{j-1}^{+}(t) & =0, j \geq 1,
\end{aligned}\right.
$$

with the initial condition $b_{0}^{+}(0)=a^{+}, b_{j}^{+}(0)=0$ for $j=1,2, \ldots, N$.

Proof. We mimic Bouclet's argument [3, Lemma 6.4]. Choose $R_{0}^{\prime}, R_{0}^{\prime \prime}>0, U_{0}^{\prime}, U_{0}^{\prime \prime} \Subset \mathbb{R}^{d-1}$ and $J_{0}^{\prime}, J_{0}^{\prime \prime} \Subset(0, \infty)$ so that

$$
\begin{array}{ll}
R_{0}>R_{0}^{\prime}>R_{0}^{\prime \prime}>R_{1}, & U_{0} \Subset U_{0}^{\prime} \Subset U_{0}^{\prime \prime} \Subset U_{1}, \\
J_{0} \Subset J_{0}^{\prime} \Subset J_{0}^{\prime \prime} \Subset J_{1}, & \sigma_{0}<\sigma_{0}^{\prime}<\sigma_{0}^{\prime \prime}<\sigma_{1} .
\end{array}
$$


For $0 \leq s, t \leq R_{1} t_{0}$ and $(r, \theta, \rho, \omega) \in \Gamma^{+}\left(R_{0}^{\prime \prime}, U_{0}^{\prime \prime}, J_{0}^{\prime \prime}, \sigma_{0}^{\prime \prime}\right)$, we consider the flow generated by $x^{+}(t)$, that is the solution to the ODE:

$$
\left\{\begin{aligned}
\left(\partial_{t} r^{+}(t, s), \partial_{t} \theta^{+}(t, s)\right) & =x^{+}\left(t, r^{+}(t, s), \theta^{+}(t, s), \rho, \omega\right), \\
\left(r^{+}(s, s), \theta^{+}(s, s)\right) & =(r, \theta) .
\end{aligned}\right.
$$

Since $x^{+}(t)=\left(\partial_{r} \Psi^{+}(t), r^{-2}\left(h^{j k}+a^{j k}\right) \partial_{\theta^{k}} \Psi^{+}(t)\right)$, by using (7.2), we have

$$
\left|\partial_{t} r^{+}(t, s)\right| \leq C\left(1+r^{+}(t, s)^{-1}|t|\right), \quad\left|\partial_{t} \theta^{+}(t, s)\right| \leq C r^{+}(t, s)^{-2}|t| .
$$

In particular,

$$
\left|\partial_{t} r^{+}(s, s)\right| \leq C, \quad\left|\partial_{t} \theta^{+}(s, s)\right| \leq C R_{0}^{\prime \prime-1} t_{0} .
$$

Therefore, a same argument as that in the proof of Lemma 5.13 implies that there exists $t_{0}>0$, independent of $R_{0}$, such that $\left(r^{+}(t, s), \theta^{+}(t, s)\right)$ is well-defined on $\Gamma^{+}\left(R_{0}^{\prime \prime}, U_{0}^{\prime \prime}, J_{0}^{\prime \prime}, \sigma_{0}^{\prime \prime}\right)$ for all $0 \leq s, t \leq R_{1} t_{0}$, and that

$$
\left|r^{+}(t, s)-r\right| \leq C|t|, \quad\left|\theta^{+}(t, s)-\theta\right| \leq t_{0} \quad \text { on } \quad \Gamma^{+}\left(R_{0}^{\prime \prime}, U_{0}^{\prime \prime}, J_{0}^{\prime \prime}, \sigma_{0}^{\prime \prime}\right) .
$$

In particular, $\left(r^{+}(t, s), \theta^{+}(t, s), \rho, \omega\right) \in \Gamma^{+}\left(R_{1}, U_{1}, J_{1}, \sigma_{1}\right)$ for all $0 \leq s, t \leq R_{1} t_{0}$. Moreover, differentiating the integral equation

$$
\left(r^{+}(t, s), \theta^{+}(t, s)\right)=(r, \theta)+\int_{s}^{t} x^{+}\left(u, r^{+}(u, s), \theta^{+}(u, s), \rho, \omega\right) d u
$$

with respect to $\partial^{\gamma}=\partial_{r}^{j} \partial_{\theta}^{\alpha} \partial_{\rho}^{k} \partial_{\omega}^{\beta}$, by using (7.2) and an induction on $|\gamma|$, we have

$$
\left|\partial^{\gamma}\left(r^{+}(t, s)-r\right)\right| \leq C_{\gamma} r^{-j-\beta}|t|, \quad\left|\partial^{\gamma}\left(\theta^{+}(t, s)-\theta\right)\right| \leq C_{\gamma} r^{-j-\beta} t_{0},
$$

for all $0 \leq s, t \leq R_{1} t_{0}$ and $(r, \theta, \rho, \omega) \in \Gamma^{+}\left(R_{0}^{\prime \prime}, U_{0}^{\prime \prime}, J_{0}^{\prime \prime}, \sigma_{0}^{\prime \prime}\right)$. We put

$$
\Phi_{+}(t, s):=\left(r^{+}(t, s), \theta^{+}(t, s), \rho, \omega\right),
$$

and define $b_{j}^{+}(t)$ by

$$
\left\{\begin{array}{l}
b_{0}^{+}(t):=a^{+}\left(\Phi_{+}(0, t)\right) e^{\int_{0}^{t} y^{+}\left(s, \Phi_{+}(s, t)\right) d s}, \\
b_{j}^{+}(t):=-\int_{0}^{t}\left(i \widehat{P}_{\kappa} b_{j-1}^{+}\right)\left(s, \Phi_{+}(s, t)\right) e^{\int_{s}^{t} y^{+}\left(u, \Phi_{+}(u, t)\right) d u} d s, \quad j=1, \ldots, N .
\end{array}\right.
$$

We remark that if we choose $t_{0}$ small enough, then (7.8) implies

$$
\begin{aligned}
\Phi_{+}(t, s)\left(\Gamma^{+}(R, U, J, \sigma)\right) & \subset \Gamma^{+}\left(R_{0}, U_{0}, J_{0}, \sigma_{0}\right), \\
\Phi_{+}(t, s)\left(\Gamma^{+}\left(R_{0}, U_{0}, J_{0}, \sigma_{0}\right)\right) & \subset \Gamma^{+}\left(R_{0}^{\prime}, U_{0}^{\prime}, J_{0}^{\prime}, \sigma_{0}^{\prime}\right), \\
\Phi_{+}(t, s)\left(\Gamma^{+}\left(R_{0}^{\prime}, U_{0}^{\prime}, J_{0}^{\prime}, \sigma_{0}^{\prime}\right)\right) & \subset \Gamma^{+}\left(R_{0}^{\prime \prime}, U_{0}^{\prime \prime}, J_{0}^{\prime \prime}, \sigma_{0}^{\prime \prime}\right) \subset \Gamma^{+}\left(R_{1}, U_{1}, J_{1}, \sigma_{1}\right),
\end{aligned}
$$

for all $0 \leq s, t \leq R_{1} t_{0} . \Phi_{+}(s, t)$ is thus well-defined on $\Gamma^{+}\left(R_{0}^{\prime}, U_{0}^{\prime}, J_{0}^{\prime}, \sigma_{0}^{\prime}\right)$, smooth on $\Gamma^{+}\left(R_{0}, U_{0}, J_{0}, \sigma_{0}\right)$, and satisfies $\Phi_{+}(s, t)=\Phi_{+}(t, s)^{-1}$. Moreover, $\operatorname{supp} b_{j}^{+}(t) \subset \Gamma^{+}\left(R_{0}, U_{0}, J_{0}, \sigma_{0}\right)$ since $\operatorname{supp} a^{+} \subset$ $\Gamma^{+}(R, U, J, \sigma)$. If we extend $b_{j}^{+}(t)$ on $\mathbb{R}^{2 d}$ so that $b_{j}^{+}(t)=0$ outside $\Gamma^{+}\left(R_{0}, U_{0}, J_{0}, \sigma_{0}\right)$, then $b_{j}^{+}(t)$ are still smooth with respect to $(r, \theta, \rho, \omega)$. Furthermore, by (2.3), (7.2) and (7.8), we see that $\left(b_{j}^{+}(t)\right)_{0 \leq t \leq R_{1} t_{0}}$ is bounded in $S_{\mathrm{sc}}\left(\Gamma^{ \pm}\left(R_{0}, U_{0}, J_{0}, \sigma_{0}\right)\right)$. Finally, a standard Hamilton-Jacobi theory shows that $b_{j}^{+}(t)$ solve (7.7) for $0 \leq t \leq R_{1} t_{0}$.

Proof of Theorem 7.1. By the construction, $B_{+}(0)=a^{+}\left(r, \theta, h D_{r}, h D_{\theta}\right) . \Psi^{+}$solves (7.1) on $\Gamma^{+}\left(R_{1}, U_{1}, J_{1}, \sigma_{1}\right)$ which contains $\Gamma^{+}\left(R_{0}, U_{0}, J_{0}, \sigma_{0}\right)$, and $b_{j}^{+}$satisfy (7.7) on the latter region. Therefore, a direct computation yields

$$
\left(h D_{s}+h^{2} \widehat{P}_{\kappa}\right) B_{+}(s)=h^{N+2} i \widehat{P}_{\kappa} b_{N}^{+}(s) .
$$

Since $\left(\widehat{P}_{\kappa} b_{N}^{+}(s)\right)_{0 \leq s \leq R t_{0}}$ is bounded in $S_{\mathrm{sc}}\left(\Gamma^{ \pm}\left(R_{0}, U_{0}, J_{0}, \sigma_{0}\right)\right),(7.1)$ implies that $I_{\mathrm{WKB}}^{+}\left(i \widehat{P}_{\kappa} b_{j}^{+}(s)\right)$ is bounded on $L^{2}\left(\mathbb{R}^{d}\right)$ uniformly with respect to $h \in(0,1]$ and $0 \leq s \leq R t_{0}$. We hence proved (7.6). 
7.3. Dispersive estimates. We here prove dispersive estimates for the WKB parametrix. Let $R_{0}>R_{1}>R_{\mathrm{WKB}}$ and $t_{\mathrm{WKB}}>0$ be as in Theorem 7.3.

Theorem 7.6. For any $\left(b^{ \pm}(t)\right)_{0 \leq \pm t \leq R_{1} t_{\mathrm{WKB}}}$ bounded in $S_{\mathrm{sc}}\left(\Omega_{s}^{ \pm}\left(R_{0}, U_{0}, J_{0}, \sigma_{0}\right)\right)$, we can write

$$
I_{\mathrm{WKB}}^{ \pm}\left(b^{ \pm}(t)\right)=U_{\mathrm{WKB}}^{ \pm}(t, h)+R_{\mathrm{WKB}}^{ \pm}(t, h),
$$

where $U^{ \pm}(t, h)$ satisfy

$$
\left\|r^{-\frac{d-1}{2}} U_{\mathrm{WKB}}^{ \pm}(t, h) r^{-\frac{d-1}{2}}\right\|_{\mathcal{L}\left(L^{1}\left(\mathbb{R}^{d}\right), L^{\infty}\left(\mathbb{R}^{d}\right)\right)} \leq C|t h|^{-d / 2},
$$

for all $0< \pm t \leq \min \left(R_{1} t_{\mathrm{WKB}}, h^{-1}\right), h \in(0.1]$. Moreover the remainder terms $R_{\mathrm{WKB}}^{ \pm}(t, h)$ are rapidly decaying with respect to $h$ : for any $N \geq 0$,

$$
\left\|R_{\mathrm{WKB}}^{ \pm}(t, h)\right\|_{\mathcal{L}\left(L^{2}\left(\mathbb{R}^{d}\right)\right)} \leq C_{N} h^{N}, \quad 0 \leq \pm t \leq \min \left(R_{1} t_{\mathrm{WKB}}, h^{-1}\right), h \in(0.1] .
$$

Here constants $C, C_{N}>0$ may be taken uniformly with respect to $h$ and $R_{1}$.

Proof. Since the proof is similar as that of Theorem 5.14, we omit details and give the sketch of the proof. We consider the outgoing case only. The distribution kernel of $I_{\mathrm{WKB}}^{+}\left(b^{+}(t)\right)$ is written in the form

$$
I_{b^{+}}(t, h)=(2 \pi h)^{-d} \int e^{\frac{i}{h} \psi^{+}\left(t, r, \theta, \rho, \omega, r^{\prime}, \theta^{\prime}\right)} b^{+}(t, r, \theta, \rho, \omega) d \rho d \omega
$$

where $\psi^{+}\left(t, r, \theta, \rho, \omega, r^{\prime}, \theta^{\prime}\right)=\Psi^{+}(t, r, \theta, \rho, \omega)-r^{\prime} \rho-\theta^{\prime} \cdot \omega$. Let $\chi_{\rho} \in C_{0}^{\infty}(\mathbb{R}), \chi_{\omega} \in C_{0}^{\infty}\left(\mathbb{R}^{d-1}\right)$ be as in the proof of Theorem 5.14, and set

$$
B^{+}(t)=\chi_{\rho}\left(\partial_{\rho} \psi^{+}\right) \chi_{\omega}\left(\partial_{\omega} \psi^{+}\right) b^{+}(t) .
$$

We then have $\left|\partial_{\rho} \psi^{+}\right|<1$ and $\left|\partial_{\omega} \psi^{+}\right|<1$ on supp $B^{+}$. We denote the operator having the kernel $I_{B^{+}}(t, h)$ by $U_{B^{+}}(t, h)$. By a same proof as that in Lemma 5.15 , we obtain that $R_{\mathrm{WKB}}^{+}(t, h)=$ $I_{\mathrm{WKB}}^{+}\left(b^{+}(t)\right)-U_{B^{+}}(t, h)$ satisfies $(7.10)$.

We next prove $(7.9)$ for $U_{\mathrm{WKB}}^{+}(t, h):=U_{B^{+}}(t, h)$. Assume $R_{1}>4$ without loss of generality. We first note that $r^{\prime} \geq R_{1} / 2$ on $\operatorname{supp} B^{+}$; otherwise, there exists $C_{0}>0$, independent of $R_{0}$, such that

$$
\left|\partial_{\rho} \psi^{+}\right| \geq r-r^{\prime}-C_{0} R_{1} t_{\mathrm{WKB}} \geq\left(1 / 2-C_{0} t_{\mathrm{WKB}}\right) R_{0}>1
$$

if $0<t_{\mathrm{WKB}}<\left(4 C_{0}\right)^{-1}$, since $r>R_{0}>R_{1}$ on $\operatorname{supp} b^{+}(t)$ and $\partial_{\rho} \psi=r-r^{\prime}+O(|t|)$ by (7.2). This contradicts the support property of $B^{+}$. We thus have

$$
r^{\prime} / 4 \leq r^{\prime}-\left|\partial_{\rho} \psi^{+}\right|-C_{0} R_{1} t_{\mathrm{WKB}} \leq r \leq r^{\prime}+\left|\partial_{\rho} \psi^{+}\right|+C_{0} R_{1} t_{\mathrm{WKB}} \leq 4 r^{\prime}
$$

on the support of $B^{+}$. It follows from (7.3) that $\psi^{+}$may be written in the form

$$
\psi^{+}\left(t, r, \theta, \rho, \nu, r^{\prime}, \theta^{\prime}\right)=\left(r-r^{\prime}\right) \rho+\left(\theta-\theta^{\prime}\right) \cdot \omega-t p(r, \theta, \rho, \omega)+Q^{+}(t, r, \theta, \rho, \omega)
$$

on $\operatorname{supp} B^{+}$, where the remainder $Q^{+}$satisfies

$$
\left|\partial_{r}^{j} \partial_{\theta}^{\alpha} \partial_{\rho}^{k} \partial_{\omega}^{\beta} Q^{+}(t, r, \theta, \rho, \omega)\right| \leq C r^{-j-|\beta|}|t| t_{\mathrm{WKB}} \quad \text { on } \quad \operatorname{supp} B^{+} .
$$

Let $\chi \in C^{\infty}\left(\mathbb{R}^{2 d}\right)$ be such that $0 \leq \chi \leq 1$, supp $\chi \subset \Gamma^{+}\left(R_{1}, U_{1}, J_{1}, \sigma_{1}\right), \chi \equiv 1$ on $\Gamma^{+}\left(R_{0}, U_{0}, J_{0}, \sigma_{0}\right)$, and that $\partial_{r}^{j} \partial_{\theta}^{\alpha} \partial_{\rho}^{k} \partial_{\omega}^{\beta} \chi=O\left(\langle r\rangle^{-j-|\beta|}\right)$. We then define

$$
\begin{aligned}
& \psi_{0}^{+}\left(t, r, \theta, \rho, \omega, r^{\prime}, \theta^{\prime}\right) \\
& :=\left(r-r^{\prime}\right) \rho+\left(\theta-\theta^{\prime}\right) \cdot \omega-t p(r, \theta, \rho, \omega)+\chi(r, \theta, \rho, \omega) Q^{+}(t, r, \theta, \rho, \omega) .
\end{aligned}
$$

Since $\psi_{0}^{+} \equiv \psi^{+}$on $\left[0, R_{1} t_{\mathrm{WKB}}\right] \times \operatorname{supp} B^{+}$, using the change of the variable $\omega \mapsto r \nu, I_{B^{+}}(t, h)$ reads

$$
I_{B^{+}}(t, h)=\frac{r^{d-1}}{(2 \pi h)^{d}} \int e^{\frac{i}{h} \psi_{0}^{+}\left(t, r, \theta, \rho, r \nu, r^{\prime}, \theta^{\prime}\right)} \tilde{B}^{+}\left(r, \theta, \rho, \nu, r^{\prime}, \theta^{\prime}\right) d \rho d \nu,
$$

where $\tilde{B}^{+}\left(r, \theta, \rho, \nu, r^{\prime}, \theta^{\prime}\right):=B^{+}\left(r, \theta, \rho, r \nu, r^{\prime}, \theta^{\prime}\right)$ is compactly supported with respect to $(\rho, \nu)$, and all derivatives of $\tilde{B}^{+}$are bounded on $\mathbb{R}^{3 d}$. Since (7.9) is obvious for $0<t<h$ (note that $\left.r^{\prime} / 4<r<4 r^{\prime}\right)$, we may assume that $h<t \leq R_{1} t_{\mathrm{WKB}}$. Set $\tilde{\psi}_{0}^{+}\left(t, r, \theta, \rho, \nu, r^{\prime}, \theta^{\prime}\right):=$ 
$t^{-1} \psi_{0}^{+}\left(t, r, \theta, \rho, r \nu, r^{\prime}, \theta^{\prime}\right)$. Choose $\chi_{1} \in C_{0}^{\infty}(\mathbb{R})$ so that $\chi_{1} \equiv 1$ on $(-1 / 2,1 / 2)$ and $\operatorname{supp} \chi_{1} \subset$ $(-1,1)$, and set

$$
\tilde{B}_{1}=\chi_{1}\left(\frac{r-r^{\prime}}{C_{1} t}\right) \tilde{B}^{+}, \quad \tilde{B}_{2}=\tilde{B}^{+}-\tilde{B}_{1},
$$

with some large $C_{1}>0$. Since $\partial_{\rho} \tilde{\psi}_{0}^{+}=\left(r-r^{\prime}\right) / t-\rho-O\left(t_{\mathrm{WKB}}\right)$, if $C_{1}>0$ is large enough, then we have $\left|\partial_{\rho} \tilde{\psi}_{0}^{+}\right| \gtrsim 1$ on $\operatorname{supp} \tilde{B}_{2}$. An integration by parts then implies that

$$
\left|\frac{1}{(2 \pi h)^{d}} \int e^{\frac{i}{h} t \tilde{\psi}_{0}^{+}\left(t, r, \theta, \rho, \nu, r^{\prime}, \theta^{\prime}\right)} \tilde{B}_{2}^{+}\left(r, \theta, \rho, \nu, r^{\prime}, \theta^{\prime}\right) d \rho d \nu\right| \leq C_{t_{\mathrm{WKB}}}|t h|^{-d / 2}
$$

for $h \leq t \leq R_{1} t_{\mathrm{WKB}}$, where $C_{t_{\mathrm{WKB}}}$ is independent of $R_{1}$. Since

$$
\partial_{\rho, \nu}^{2} p(r, \theta, \rho, r \nu)=\left(\begin{array}{cc}
1 & 0 \\
0 & h^{j k}(\theta)+a^{j k}(r, \theta)
\end{array}\right)
$$

is bounded from above and below on $\mathbb{R}^{d}$, we obtain

$$
\left|\partial_{\rho}^{k} \partial_{\nu}^{\beta} \tilde{\psi}_{0}^{+}\left(t, r, \theta, \rho, \nu, r^{\prime}, \theta^{\prime}\right)\right| \leq C \quad \text { for } \quad|k+\beta| \geq 2,
$$

uniformly with respect to $\left(r, \theta, \rho, \omega, r^{\prime}, \theta^{\prime}\right) \in \mathbb{R}^{3 d}$ and $0<t \leq R_{1} t_{\mathrm{WKB}}$. Moreover, if $t_{\mathrm{WKB}}>0$, which can be taken uniformly with respect to $R_{1}$, is small enough, then we have

$$
\left|\operatorname{det} \partial_{\rho, \nu}^{2} \tilde{\psi}_{0}^{+}\left(t, r, \theta, \rho, \nu, r^{\prime}, \theta^{\prime}\right)\right| \gtrsim 1, \quad\left(r, \theta, \rho, \omega, r^{\prime}, \theta^{\prime}\right) \in \mathbb{R}^{3 d}, 0<t \leq R_{1} t_{\mathrm{WKB}} .
$$

By a same argument as that in Section 5 , we can apply the stationary phase theorem, and have the assertion since $r^{\prime} / 4<r<4 r^{\prime}$ on the support of $\tilde{B}^{+}$.

\section{Proof of Theorem 3.3}

We here prove Theorem 3.3. We only consider the case $t \geq 0$, and the proof for the case $t \leq 0$ is similar. Recall that $A_{h}=\sum_{\kappa} \mathrm{Op}_{\kappa}^{p r}\left(a_{\kappa, h}\right)$ is a sum of properly supported $h$-PDO's. By (2.9), (2.11) and their adjoint estimates with respect to $\widehat{G} d z$, we have

$$
\left\{\begin{array}{l}
\left\|r^{-\frac{d-1}{2}} A_{h}\right\|_{\mathcal{L}\left(L^{2}(\widehat{M}), L^{\infty}(\widehat{M})\right)}+\left\|A_{h}^{*} r^{-\frac{d-1}{2}}\right\|_{\mathcal{L}\left(L^{1}(\widehat{M}), L^{2}(\widehat{M})\right)} \leq C h^{-\frac{d}{2}}, \\
\left\|r^{-\frac{d-1}{2}} A_{h} r^{\frac{d-1}{2}}\right\|_{\mathcal{L}\left(L^{\infty}(\widehat{M})\right)}+\left\|r^{\frac{d-1}{2}} A_{h}^{*} r^{-\frac{d-1}{2}}\right\|_{\mathcal{L}\left(L^{1}(\widehat{M})\right)} \leq C,
\end{array}\right.
$$

uniformly with respect to $h \in(0,1]$.

Choose $R_{0}>0$ and $\varepsilon_{0}>0$ so that Theorems 5.10, 5.14, 6.1, 7.3 and 7.6 hold for all $R \geq R_{0}$ and $0<\varepsilon \leq \varepsilon_{0}$, and let $N \geq d$.

We first prove (3.5). A standard symbolic calculus implies that $\mathrm{Op}_{\kappa}\left(b_{s}^{+}\right)^{*}$ can be replaced by $\mathrm{Op}_{\kappa}\left(\tilde{b}_{s}^{+}\right)$modulo a smoothing term $O\left(h^{N+1}\right)$ on $L^{2}(\widehat{M})$, where $\tilde{b}_{s}^{+} \in S_{\mathrm{sc}}\left(\Omega_{s}^{+}\left(R_{2}, \tilde{U}_{\kappa}, J, \varepsilon\right)\right)$. Moreover, $\mathrm{Op}_{\kappa}\left(a_{s}^{+}\right)-\mathrm{Op}_{\kappa}^{p r}\left(a_{s}^{+}\right)$has the $\mathcal{L}\left(L^{2}(\widehat{M})\right)$-norm of order $h^{N+1}$ by (2.7). Therefore, Theorems $5.10,5.14$ with $R=R_{2}$ and (2.11) with $(q, s)=(\infty,(d-1) / 2)$ imply that $\mathrm{Op}_{\kappa}\left(a_{s}^{+}\right) e^{-i t h \widehat{P}} \mathrm{Op}_{\kappa}\left(b_{s}^{+}\right)^{*}$ can be brought to the form

$$
U_{s}^{+}(t, h, N)+h^{N+1} R_{s}^{+}(t, h, N),
$$

where $U_{s}^{+}(t, h, N)$ and $R_{s}^{+}(t, h, N)$ satisfy

$$
\begin{aligned}
\left\|r^{-\frac{d-1}{2}} U_{s}^{+}(t, h, N) r^{-\frac{d-1}{2}}\right\|_{\mathcal{L}\left(L^{1}(\widehat{M}), L^{\infty}(\widehat{M})\right)} & \leq C_{N}|t h|^{-\frac{d}{2}}, \\
\left\|R_{s}^{+}(t, h, N)\right\|_{\mathcal{L}\left(L^{2}(\widehat{M})\right)} & \leq C_{N},
\end{aligned}
$$

uniformly with respect to $h \in(0,1]$ and $0< \pm t \leq h^{-1}$. By using (8.1), we obtain (3.5) since $1 \leq|t h|^{-\frac{d}{2}}$ for $0<t \leq h^{-1}$.

Next, let $R_{2} \geq R_{0}$ and $R_{1}>0$ so that $2 R_{1}>R_{2}>R_{1}$. Then, there exists $t_{\mathrm{WKB}}>0$, independent of $R_{2}$, such that Theorems 7.3 and 7.6 with $R=R_{2}$ hold for $0<t \leq R_{2} t_{\mathrm{WKB}} / 2$. By a same argument as above, we obtain (3.4) with $t_{0}=t_{\mathrm{WKB}} / 2$.

For any $t_{1}>0$, a same argument as above and Theorem 6.1 imply

$$
\left\|\mathrm{Op}_{\kappa}\left(a_{l}^{+}\right) e^{-i t h \widehat{P}} \operatorname{Op}_{\kappa}\left(b_{l}^{+}\right)^{*}\right\|_{\mathcal{L}\left(L^{2}(\widehat{M})\right)} \leq C_{N} h^{N+1},
$$


for $h \in(0,1]$ and $R_{2} t_{1} \leq t \leq h^{-1}$. Combining this estimate with (8.1), we obtain (3.6). We complete the proof.

\section{Appendix A. Proof of Theorem 1.2}

We here give the sketch of the proof of Theorem 1.2. It is sufficient to prove that, for any $K \Subset M, \chi_{K} \in C_{0}^{\infty}(M)$ with $\chi_{K} \equiv 1$ on $K$ and admissible pair $(p, q)$, the following estimate holds under the nontrapping condition:

$$
\left\|\chi_{K} e^{-i t P} u_{0}\right\|_{L^{p}\left([0,1] ; L^{q}(M)\right)} \leq C\left\|u_{0}\right\|_{L^{2}(M)} .
$$

We mimic the Bouclet-Tzvetkov argument [4, Section 5 and 6].

Proposition A.1. Let $\varphi \in C_{0}^{\infty}((0, \infty))$. Then there exist $t_{0}>0$ and $C>0$ such that for all $h \in(0,1]$ and admissible pair $(p, q)$,

$$
\left\|\chi_{K} \varphi\left(h^{2} P\right) e^{-i t P} u_{0}\right\|_{L^{p}\left(\left[0, t_{0} h\right] ; L^{q}(M)\right)} \leq C\left\|u_{0}\right\|_{L^{2}(M)} .
$$

Proof. Let $\left\{\kappa: V_{\kappa} \rightarrow U_{\kappa}\right\}_{\kappa}$ be a finite atlas on $K$ and $\left\{\psi_{\kappa}\right\}_{\kappa} \subset C_{0}^{\infty}(M)$ be a partition of unity subordinate to $\left\{V_{\kappa}\right\}$. Let $\tilde{\psi}_{\kappa} \in C_{0}^{\infty}(M)$ so that $\tilde{\psi}_{\kappa} \equiv 1$ on a neighborhood of $\operatorname{supp} \psi$. For a symbol $a \in C_{0}^{\infty}\left(U_{\kappa} \times \mathbb{R}^{d}\right)$, we define $\operatorname{Op}_{\kappa}(a) u=\kappa^{*}\left(a\left(z, h D_{z}\right) \kappa_{*}\left(\tilde{\psi}_{\kappa} u\right)\right)$. Since $a$ has a compact support with respect $z$ and $\xi$, the Schur lemma implies that for $1 \leq q \leq r \leq \infty$ and $h \in(0,1]$,

$$
\left\|a\left(z, h D_{z}\right)\right\|_{\mathcal{L}\left(L^{q}\left(\mathbb{R}^{d}\right), L^{r}\left(\mathbb{R}^{d}\right)\right)} \leq C h^{-d(1 / q-1 / r)} .
$$

By a same argument as that in [6, Section 2] which studied the case of compact manifolds without boundaries (see also [1]), there exist symbols $a_{\kappa, h} \in C_{0}^{\infty}\left(U_{\kappa} \times p_{\kappa}^{-1}(\operatorname{supp} \varphi)\right)$ such that we can approximate $\chi_{K} \varphi\left(h^{2} P\right)$ by $\sum_{\kappa} \mathrm{Op}_{\kappa}\left(a_{\kappa, h}\right)$ up to $O\left(h^{N}\right)$ on $L^{2}(M)$ for any $N \geq 0$. In particular if we obtain the following estimate:

$$
\left\|\mathrm{Op}_{\kappa}(b) \mathrm{Op}_{\kappa}(a) e^{-i t P} u_{0}\right\|_{L^{p}\left(\left[0, t_{0} h\right] ; L^{q}(M)\right)} \leq C\left\|u_{0}\right\|_{L^{2}(\widehat{M})}, \quad h \in(0,1],
$$

for any $a, b \in C_{0}^{\infty}\left(U_{\kappa} \times p_{\kappa}^{-1}(\operatorname{supp} \varphi)\right)$, then a same argument as that in Section 3 implies (A.2). (A.4) follows from (A.3), the $T T^{*}$-argument and the WKB parametric approximation of the propagator $e^{-i t h P} \mathrm{Op}_{\kappa}(a)$ for $0 \leq t \leq t_{0}$ with sufficiently small $t_{0}>0$. The construction of the WKB parametrix is basically well known and its proof is similar as that in the case of elliptic operators on the Euclidean space. We refer to [18] for details. Dispersive estimates can be proved similarly to the case of compact manifolds without boundaries [6].

Proof of (A.1). The Duhamel formula implies

$$
\varphi\left(h^{2} P\right) \chi_{K} e^{-i t P} u_{0}=e^{-i t P} \varphi\left(h^{2} P\right) \chi_{K} u_{0}-i \int_{0}^{t} e^{-i(t-s) P} \varphi\left(h^{2} P\right)\left[P, \chi_{K}\right] e^{-i s P} u_{0} d s .
$$

By Theorem 1.1, Proposition A.1 and a same argument as that in [4, Section 5 and 6], we obtain

$$
\begin{aligned}
& \left\|\varphi\left(h^{2} P\right) \chi_{K} e^{-i t P} u_{0}\right\|_{L^{p}\left([0,1] ; L^{q}(M)\right)} \\
& \lesssim\left\|\varphi\left(h^{2} P\right) \chi_{K} u_{0}\right\|_{L^{2}(M)}+h^{-1 / 2}\left\|\varphi\left(h^{2} P\right) \chi_{K} e^{-i t P} u_{0}\right\|_{L^{2}\left([0,1] ; L^{2}(M)\right)} \\
& \quad+h^{1 / 2}\left\|\varphi\left(h^{2} P\right)\left[P, \chi_{K}\right] e^{-i s P} u_{0}\right\|_{L^{2}\left([0,1] ; L^{2}(M)\right)} \\
& \lesssim\left\|\varphi\left(h^{2} P\right) u_{0}\right\|_{L^{2}(M)}+h^{1 / 2}\left\|u_{0}\right\|_{L^{2}(M)}+\left\|\chi_{K} \varphi\left(h^{2} P\right) e^{-i t P} u_{0}\right\|_{L^{2}\left([0,1] ; H^{1 / 2}(M)\right)},
\end{aligned}
$$

where $H^{1 / 2}(M)$ is the Sobolev space with the norm $\left\|(1+P)^{1 / 4} \cdot\right\|_{L^{2}(M)}$. In the last line we used inequalities

$$
\begin{aligned}
& \left\|\left[\varphi\left(h^{2} P\right), \chi_{K}\right]\right\|_{\mathcal{L}\left(L^{2}(M)\right)} \lesssim h,\left\|\left[\varphi\left(h^{2} P\right),\left[P, \chi_{K}\right]\right]\right\|_{\mathcal{L}\left(L^{2}(M)\right)} \lesssim 1, \\
& \left\|\left[P, \chi_{K}\right] \tilde{\chi}_{K} \varphi\left(h^{2} P\right)\right\|_{\mathcal{L}\left(H^{1 / 2}(M), H^{-1 / 2}(M)\right)} \lesssim 1,
\end{aligned}
$$

where $\tilde{\chi}_{K} \in C_{0}^{\infty}(M)$ is a cut-off function so that $\tilde{\chi}_{K} \equiv 1$ on supp $\chi_{K}$. These inequalities follow from the pseudodifferential approximations of $\chi_{K} \varphi\left(h^{2} P\right)$ and $\left(1-\chi_{K}\right) \varphi\left(h^{2} P\right)$ such as Lemma 2.4 (see also [1]) and the symbolic calculus. 
We now use the nontrapping condition on the metric. Under the nontrapping condition, Cardoso-Vodev [7] proved that for sufficiently large $\lambda>0$,

$$
\left\|\chi_{K}(P-\lambda \pm i 0)^{-1} \chi_{K}\right\|_{\mathcal{L}\left(L^{2}(M)\right)} \lesssim\langle\lambda\rangle^{-1 / 2} .
$$

By the abstract Kato smooth perturbation theory, this resolvent estimate implies the local smoothing effect:

$$
\left\|\chi_{K} e^{-i t P} u_{0}\right\|_{L^{2}\left([0,1] ; H^{1 / 2}(M)\right)} \lesssim\left\|u_{0}\right\|_{L^{2}(M)} .
$$

Using this estimate, we obtain

$$
\left\|\varphi\left(h^{2} P\right) \chi_{K} e^{-i t P} u_{0}\right\|_{L^{p}\left([0,1] ; L^{q}(M)\right)} \lesssim\left\|\varphi\left(h^{2} P\right) u_{0}\right\|_{L^{2}(M)}+h^{1 / 2}\left\|u_{0}\right\|_{L^{2}(M)}
$$

uniformly with respect to $h \in(0,1]$. Applying the Littlewood-Paley decomposition proved by [2], we obtain (A.1) and conclude the proof.

\section{REFERENCES}

[1] Bouclet, J.- M. Semi-classical calculus on non compact manifolds with ends and weighted $L^{p}$ estimates. To appear in Ann. Inst. Fourier.

[2] Bouclet, J.- M. (2010). Littlewood-Paley decompositions on manifolds with ends. Bull. Soc. Math. France. 138:1-37

[3] Bouclet, J.- M. Strichartz estimates on asymptotically hyperbolic manifolds. To appear in Analysis and PDE.

[4] Bouclet, J.- M., Tzvetkov, N. (2007). Strichartz estimates for long range perturbations. Amer. J. Math. 129:1565-1609.

[5] Bouclet, J.- M., Tzvetkov, N. (2008). On global Strichartz estimates for non trapping metrics. J. Funct. Analysis. 254:1661-1682.

[6] Burq, N., Gérard, P., Tzvetkov, N. (2004). Strichartz inequalities and the nonlinear Schrödinger equation on compact manifolds. Amer. J. Math. 126:569-605.

[7] Cardoso, F., Vodev, G. (2002). Uniform estimates of the resolvent of the Laplace-Beltrami operator on infinite volume Riemannian manifolds, II. Ann. Henri Poincaré. 3:673-691.

[8] Doi, S. (1996). Smoothing effects of Schrödinger evolution groups on Riemannian manifolds. Duke Math. J. 82:679-706.

[9] Fujiwara, D. (1980). Remarks on convergence of the Feynman path integrals. Duke Math. J. 47:559-600.

[10] Ginibre, J., Velo, G. (1985). The global Cauchy problem for the non linear Schrödinger equation. Ann. lHP-Analyse non linéaire. 2:309-327.

[11] Hassell, A., Tao, T., Wunsch, J. (2006). Sharp Strichartz estimates on non-trapping asymptotically conic manifolds. Amer. J. Math. 128:963-1024.

[12] Isozaki, H., Kitada, H. (1985). Modified wave operators with time independent modifiers. J. Fac. Sci. Univ. Tokyo. 32:77-104.

[13] Journé, J.- L., Soffer, A., Sogge, C. D. (1991). Decay estimates for Schrödinger operators. Comm. Pure Appl. Math. 44:573-604.

[14] Keel, M., Tao, T. (1998). Endpoint Strichartz Estimates. Amer. J. Math. 120:955-980.

[15] Kumano-go, H. (1980). Pseudo-Differential Operators. MIT Press.

[16] Marzuola, J., Metcalfe, J., Tataru, D. (2008). Strichartz estimates and local smoothing estimates for asymptotically flat Schrödinger equations. J. Funct. Analysis. 255:1497-1553.

[17] Robbiano, L., Zuily, C. (2005). Strichartz estimates for Schrödinger equations with variable coefficients. Mém. SMF. Math. Fr. (N.S.). 101-102.

[18] Robert, D. (1987). Autour de l' approximation semi-classique. Progr. Math. 68 Birkhäuser, Basel.

[19] Schlag, W. (2007). Dispersive estimates for Schrödinger operators: a survey. Mathematical aspects of nonlinear dispersive equations, Ann. of Math. Stud. Princeton Univ. Press, Princeton, NJ. 163:255-285

[20] Staffilani, G., Tataru, D. (2002). Strichartz estimates for a Schrödinger operator with non smooth coefficients. Comm. PDE. 27:1337-1372.

[21] Strichartz, R. (1977). Restrictions of Fourier transforms to quadratic surfaces and decay of solutions of wave equations. Duke Math. J. 44:705-714.

[22] Tataru, D. (2008). Parametrices and dispersive estimates for Schrödinger operators with variable coefficients. Amer. J. Math. 130:571-634.

[23] Yajima, K. (1987). Existence of solutions for Schrödinger evolution equations. Comm Math. Phys. 110:415426.

[24] Yajima, K. (1995). The $W_{k, p}$-continuity of wave operators for Schrödinger operators. J. Math. Soc. Japan. 47:551-581. 
[25] Yajima, K. (2005). Dispersive estimates for Schrödinger equations with threshold resonance and eigenvalue. Comm. Math. Phys. 259:475-509. 